\title{
Multi-objective robust design of helical milling hole quality on AISI H13 hardened steel by normalized normal constraint coupled with robust parameter design
}

Robson Bruno Dutra Pereira ${ }^{*}$, Laila Alves da Silva ${ }^{2}$, Carlos Henrique Lauro ${ }^{1}$, Lincoln Cardoso Brandão $^{1}$, João Roberto Ferreira ${ }^{2}$, J. Paulo Davim ${ }^{3}$

*Corresponding author

${ }^{1}$ Department of Mechanical Engineering - Industrial Engineering, Federal University of São João del Rei (UFSJ), 170 Frei Orlando Square, São João del Rei, MG 36880-000, Brazil

${ }^{2}$ Institute of Industrial Engineering and Management, Federal University of Itajubá (UNIFEI), 1303 BPS Avenue, Itajubá, MG 37500-903, Brazil

${ }^{3}$ Department of Mechanical Engineering, University of Aveiro, Campus Santiago, 3810-193 Aveiro, Portugal

\begin{abstract}
Helical milling is a hole-making process which has been applied in hardened materials. Due to the difficulties on achieving high-quality boreholes in these materials, the influence of noise factors, and multi-quality performance outcomes, this work aims the multi-objective robust design of hole quality on AISI H13 hardened steel. Experiments were carried out through a central composite design considering process and noise factors. The process factors were the axial and tangential feed per tooth of the helix, and the cutting velocity. The noise factors considered were the tool overhang length, the material hardness and the borehole height of measurement. Response models were obtained through response surface methodology for roughness and roundness outcomes. The models presented good explanation of data variability and good prediction capability. Mean and variance models were derived through robust parameter design for all responses. Similarity analysis through cluster analysis was realised, and average surface roughness and total roundness were selected to multi-objective optimisation. Mean square error optimisation was performed to achieve bias and variance minimization. Multi-objective optimisation through normalized normal constraint was performed to achieve a robust Pareto set for the hole quality outcomes. The normalized normal constraint optimisation results outperformed the results of other methods in terms of evenness of the Pareto solutions and number of Pareto optimal solutions. The most compromise solution was selected considering the lowest Euclidian distance to the utopia point in the normalized space. Individual and moving range control charts were used to confirm the robustness achievement with regard to noise factors in the most compromise Pareto optimal solution. The methodology applied for robust modelling and optimisation of helical milling of AISI H13 hardened steel was confirmed and may be applied to other manufacturing processes.
\end{abstract}

\section{Keywords}

Helical milling; AISI H13 hardened steel; Multi-objective robust optimization; Robust parameter design; Normalized normal constraint method. 


\begin{tabular}{|l|l|}
\hline EDM & electrical-discharge machining \\
\hline CAM & computer-aided manufacturing \\
\hline CFRP & carbon fiber reinforced plastic \\
\hline DOE & design of experiments \\
\hline RSM & response surface methodology \\
\hline CCD & central composite design \\
\hline RPD & robust parameter design \\
\hline SNR & signal-to-noise ratio \\
\hline MSE & mean square error \\
\hline NNC & normalized normal constraint \\
\hline TCP & tool centre point \\
\hline OLS & ordinary least squares \\
\hline WLS & weighted least squares \\
\hline WS & weighted sum \\
\hline NBI & normal boundary intersection \\
\hline I/MR & individual values/moving range \\
\hline SQP & sequential quadratic programming \\
\hline LOF & lack-of-fit \\
\hline
\end{tabular}

\section{Nomenclature}

\begin{tabular}{|l|l|}
\hline$v_{f}$ & helical feed velocity [mm/min] \\
\hline$v_{f h a}$ & axial feed velocity of the helix [mm/min] \\
\hline$v_{f h t}$ & tangential feed velocity of the helix [mm/min] \\
\hline$f_{z a}$ & axial feed per tooth of the helix [mm/tooth] \\
\hline$z$ & number of teeth \\
\hline$n$ & spindle rotation speed [rev/min] \\
\hline$v_{f t}$ & tangential feed velocity [mm/min] \\
\hline$D_{b}$ & borehole diameter [mm] \\
\hline$D_{h}$ & helix diameter [mm] \\
\hline$f_{z t}$ & tangential feed per tooth of the helix [mm/tooth] \\
\hline$a_{p}$ & axial depth of cut [mm] \\
\hline$\varphi$ & tool contact angle $\left[{ }^{\circ}\right]$ \\
\hline$a_{p}{ }^{*}$ & maximum depth of cut $[\mathrm{mm}]$ \\
\hline$\alpha$ & helix angle $\left[{ }^{\circ}\right]$ \\
\hline$a_{e}$ & radial depth of cut $[\mathrm{mm}]$ \\
\hline $\mathbf{x}$ & vector of process variables \\
\hline$x_{i}$ & $i$-th process factor, $i=1, \ldots, k$ \\
\hline
\end{tabular}




\begin{tabular}{|c|c|}
\hline $\mathbf{z}$ & vector of noise variables \\
\hline$z_{j}$ & $j$-th noise factor, $j=1, \ldots, r$ \\
\hline$\hat{y}(\mathbf{x}, \mathbf{z})$ & Response model \\
\hline$\beta_{0}$ & Intercept of response model \\
\hline$\beta$ & vector of linear coefficients of process factors \\
\hline$\beta_{i}$ & $i$-th linear coefficient of process factors, $i=1, \ldots, k$ \\
\hline B & matrix of second-order coefficients of process factors \\
\hline$\beta_{i i}$ & $i$-th quadractic coefficient of process factors, $i=1, \ldots, k$ \\
\hline$\beta_{i j}$ & $i j$-th interaction coefficient of process factors, $i \neq \mathrm{j}$ \\
\hline$\gamma$ & vector of linear coefficients of noise factors \\
\hline$\gamma_{j}$ & $j$-th linear coefficient of noise factors, $j=1, \ldots, r$ \\
\hline$\Delta$ & matrix of process $\times$ noise interaction terms \\
\hline$\delta_{i j}$ & $i j$-th process $\times$ noise interaction, $\forall i, j$ \\
\hline$\varepsilon$ & experimental error \\
\hline $\mathbf{V}$ & variance matrix of noise factors \\
\hline$\sigma_{z j}^{2}$ & variance of the $j$-th noise factor, $j=1, \ldots, r$ \\
\hline$\underset{\mathbf{z}, \mathbf{z}}{E[}[y(\mathbf{x}, \mathbf{z})]$ & mean model \\
\hline $\operatorname{Var}[y(\mathbf{x}, \mathbf{z})]$ & variance model \\
\hline$\hat{\mathbf{l}}(\mathbf{x})$ & slope in the direction of noise factors \\
\hline $\mathbf{X}^{*}$ & general design matrix of the response model \\
\hline$\beta^{*}$ & general coefficient vector of the response model \\
\hline $\mathrm{C}^{*}$ & variance-covariance matrix of $\mathbf{X}^{*}$ \\
\hline $\mathbf{W}^{*}$ & general matrix of weigths for WLS estimates of the response model \\
\hline$M S E$ & mean square error equation \\
\hline$T_{y}$ & target of the mean model \\
\hline $\mathbf{Y}$ & matrix of responses \\
\hline$y_{i j}$ & $i$-th observation of the $j$-th response, $i=1, \ldots, N, j=1, \ldots, m$. \\
\hline $\mathbf{P}$ & proximity matrix \\
\hline$p_{r s}$ & proximity between $y_{r}$ and $y_{s}$ \\
\hline$d_{r s}^{2}$ & quadratic distance \\
\hline$\Psi$ & metric of interest for the quadratic distance \\
\hline$s_{i i}$ & variance of $i$-th variable \\
\hline$S_{r s}$ & similarity between $\mathbf{y}_{r}$ e $\mathbf{y}_{s}$ \\
\hline$d_{r s}$ & dissimilarity between $\mathbf{y}_{r}$ e $\mathbf{y}_{s}$ \\
\hline$r_{r s}$ & correlation coefficient between $\mathbf{y}_{r}$ e $\mathbf{y}_{s}$ \\
\hline $\mathbf{f}(\mathbf{x})$ & vector of objective functions \\
\hline$f_{i}$ & $i$-th objective function \\
\hline$m$ & number of objective functions \\
\hline $\mathbf{x}$ & vector of control factors \\
\hline $\mathbf{f}_{i}^{*}$ & $i$-th anchor point \\
\hline $\mathbf{f}^{\mathrm{U}}$ & utopia point \\
\hline $\mathbf{f}^{\mathrm{N}}$ & nadir point \\
\hline $\mathbf{f}^{\mathrm{PN}}$ & pseudo-nadir point \\
\hline$\overline{\mathbf{f}^{U}}$ & normalized utopia point \\
\hline
\end{tabular}




\begin{tabular}{|l|l|}
\hline$\overline{\mathbf{f}}^{\mathrm{PN}}$ & normalized pseudo-nadir point \\
\hline$\overline{\mathbf{f}}$ & normalized vector of objective functions \\
\hline $\bar{f}_{i}$ & $i$-th normalized objective function \\
\hline $\mathbf{\Phi}$ & pay-off matrix \\
\hline$\overline{\mathbf{\Phi}}$ & normalized pay-off matrix \\
\hline$\overline{\mathbf{Q}}_{\mathbf{i j}}$ & point in the utopia line \\
\hline$\overline{\mathbf{N}}_{\mathrm{r}}$ & utopia line vector \\
\hline$\overline{\mathbf{f}}^{*}$ & Pareto optimal solution \\
\hline$\overline{\mathbf{f}}_{\mathrm{i}}^{*}$ & i-th normalized anchor point \\
\hline$w_{i j}$ & weigths assigned to the objective functions \\
\hline$n_{s u b}$ & number of subproblems \\
\hline$\delta_{r}$ & spacing among the points in the utopia line vector \\
\hline$\eta_{r}$ & number of points in the utopia line vector \\
\hline $\bar{d}_{j}$ & normalized Euclidian distance between the adjacent Pareto solutions \\
\hline$\overline{\mathbf{d}}$ & $\begin{array}{l}\text { vector of Euclidian distance between the adjacent Pareto solutions in } \\
\text { the normalized space }\end{array}$ \\
\hline$C V_{\overline{\mathbf{d}}}$ & coefficient of variation of $\overline{\mathbf{d}}$ \\
\hline$\sigma_{\overline{\mathbf{d}}}$ & standard deviation of $\overline{\mathbf{d}}$ \\
\hline$\mu_{\overline{\mathbf{d}}}$ & mean of $\overline{\mathbf{d}}$ \\
\hline$d_{j+}$ & Euclidian distance of each Pareto optimal solution $\overline{\mathbf{f}}_{j}^{*}$ to the utopia \\
& point $\overline{\mathbf{f}}^{\mathrm{U}}$ in the normalized space \\
\hline$a_{p(m a x)}$ & maximum axial cutting depth of the cutting tool \\
\hline$h_{d}$ & material hardness \\
\hline$l_{t o}$ & tool overhang length \\
\hline$l_{b}$ & borehole height \\
\hline$R_{a}$ & average surface roughness \\
\hline$R_{z}$ & maximum surface roughness \\
\hline$R o n_{t}$ & total roundness \\
\hline$C y l_{t}$ & total cylindricity \\
\hline$\alpha$ & significance level \\
\hline$\Omega$ & experimental region \\
\hline$\rho$ & radius of the spherical region \\
\hline$n_{f}$ & number of factorial points \\
\hline$n_{c}$ & number of centre points \\
\hline$n_{a}$ & number of axial points \\
\hline$R^{2}$ & coefficient of determination \\
\hline$R_{a d j_{j}}{ }^{2}$ & adjusted coefficient of determination \\
\hline$R_{a d j}{ }^{2}$ & prediction coefficient of determination \\
\hline & \\
\hline
\end{tabular}




\section{Introduction}

Molds and dies are important but complex tools for forming processes. Molds and dies demand evolves requesting products with better quality and high complexity, besides, reduction of time to market response and costs [1]. In this sense, molds and dies industry present important impact in the competitiveness of the forming processes. In a mold manufacturing it is estimated that $65 \%$ of the costs are due to finishing and semi-finishing processes by machining $[2,3]$. Between these processes, hole-making spends from $25 \%$ to $50 \%$ of the cycle time and $33 \%$ of the total number of operations, requesting reliability due to the high added value to the part being processed $[4,5]$.

Hard milling has captured the attention of manufacturers of the molds and dies industry. In this field materials such as AISI P20, H13 and others are commonly cut. Traditionally, core and cavities of molds and dies are removed in the hardened state using electrical-discharge machining (EDM). Nowadays, new toolpaths are available to perform hard milling of these materials. The hardness of these materials can range from $45 \mathrm{HRC}$ to as hard as $64 \mathrm{HRC}$ [6]. To allow the adoption of new technologies for hard milling, computer-aided manufacturing (CAM) software developers have programmed special toolpaths so that tool deflection in milling sculptured surfaces can be compensated for [7]. For hole-making and cavities opening through milling, a helical milling path is an option which ensures the milling advantages to the detriment of hard drilling difficulties.

In helical milling, the tool proceeds a helical path concomitant to the tool rotation around its own axis. Due to the helical path and the use of a mill instead of a drill, the helical milling presents several advantages with regard to conventional drilling. In helical milling, holes with different diameters may be obtained with the same tool throughout the helical diameter adjustment, enabling tool inventory reduction and setup time economy. In this process, the material removal is realized by frontal and peripheral cutting edges continuously and discontinuously, respectively, while in conventional drilling the material removal is continuous through the frontal cutting edges [8]. In helical milling low cutting force levels in the axial direction are developed due to the helical trajectory, due to the tool geometry and the material removal aspects [9]. Easy chip evacuation and modern lubri-cooling techniques application, such as minimum quantity lubrication and air cooling, are allowed due to the offset between tool and borehole [10].

Borehole quality is generally assessed through roughness and roundness outcomes [11]. Improved dimensional, geometrical and microgeometrical borehole quality are obtained through helical milling [9] due to the helical path, low cutting forces, discontinuous material removal through peripheral cut and easy chip evacuation. Correction of dimension deviation may be realized through 
the adjustment of the helical diameter [12]. Tool wear may be monitored, since it occurs progressively, allowing tool life prediction and tool replacement [9]. A high quality of borehole entrance and exit with low burr levels and fracture in these regions are achieved [13]. In carbon fiber reinforced plastic (CFRP), low delamination levels are obtained through hole-making with helical milling technique [14]. Through helical milling it is possible to obtain finished boreholes in just one operation, avoiding reaming operations [15]. Due to these advantages, helical milling has been consolidating as a sustainable hole-making process. Some of the advantages of helical milling process are illustrated in Figure 1.

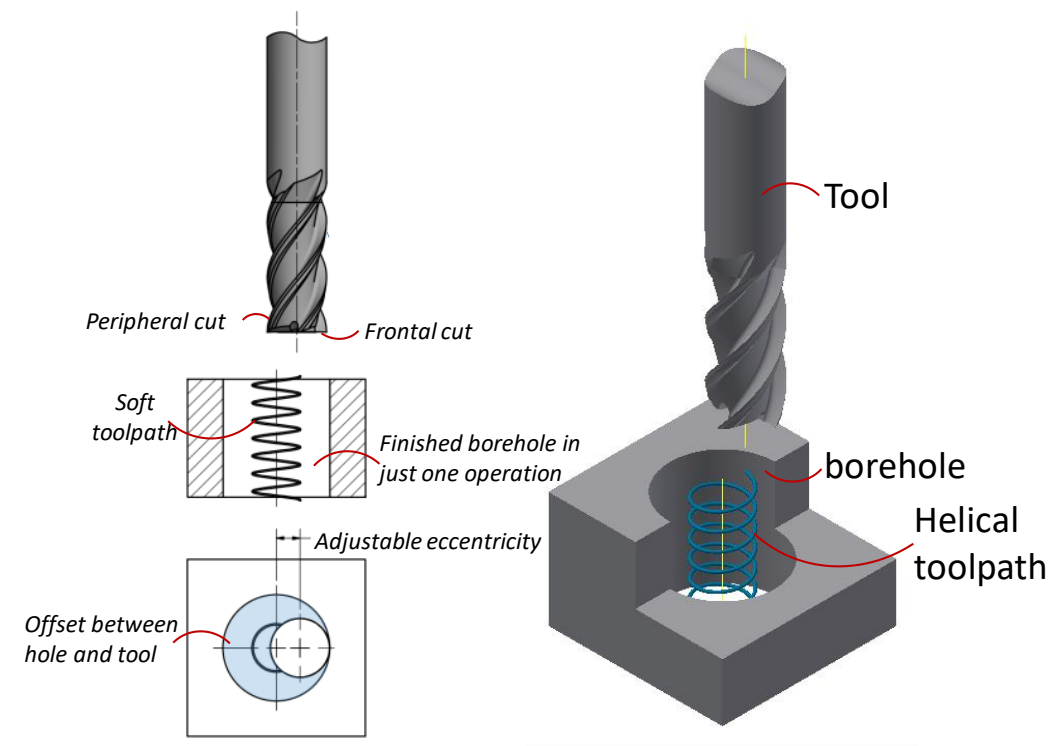

Figure 1. Helical milling features

Helical milling has been widely applied for hole-making in difficult-to-cut materials, such as titanium alloys and CFRP [16]. There are still few studies about helical milling in hardened steels. Some important results in this field are the case of helical milling of AISI D2 steel with 60 HRC [9], AISI D3 steel with 55 HRC [17] and AISI 4340 steel from 34 to 45 HRC [18].

To study the helical milling in hardened materials presents itself as a possibility of increasing competitiveness of molds and die industry due to the difficulties of drilling these materials. Efforts have been made to shorten entire process chains by direct hard machining of components, aiming to reduce production time and to substitute conventional, time and resource intensive inflexible grinding and electrical discharge machining processes [19]. As Iyer et al. [9] highlights, the advantage on manufacturing molds and dies with the workpiece hardened, instead of roughing the material in its soft state followed by heat treatment and finishing by grinding or electrical discharge machining (EDM), comes from the finishing operations with intensive labour and logistical needs, 
apart from guaranteeing a higher quality product eliminating the errors associated to heat treatment and several setups. Due to the lack of experimental studies about helical milling in hardened steels, there are scientific aspects poorly explored. Statistical and computational methods may be applied to achieve high-quality boreholes in these materials, bringing economic, social and environmental benefits to the whole chain of tool and die industry.

Process modelling through the design of experiments (DOE) aims to find an approximation for the response with regard to control factors. The response surface methodology (RSM) is one DOE methodology widely applied for modelling and optimization, including a set of tools for building and exploring empirical models [20]. To achieve these aims, designed experiments must be conducted. Among the available options, the central composite design is an interesting experimental design enabling sequential experimentation, curvature region search, economy in the experimentation, possibility of getting first and second order models, besides, low prediction variance [21].

Robust optimisation deals with uncertainties in the input data [22]. In these problems, it is desired the "robust solution" achievement, when a solution is referred as robust if its near-optimal under distinct scenarios of the input data; and "model robustness" if the solution is nearly feasible in all scenarios [23]. More specifically, Taguchi methods aim the robust design of a process or product with minimal costs and, especially, low sensitivity to fluctuations of uncontrollable variables [24]. Taguchi considers the problem on finding optimal levels of controllable factors which optimize the response taking into consideration the sensitiveness of the optimal solution obtained with regard to the noise variables which affect the process under the experimental region. Noise factors are those which affect the response and cannot be controlled by the engineer at production level [25]. The robust parameter design (RPD), aims to find levels of control factors which makes the optimal level of the response insensitive with regard to control factors variability [26,27]. The reason for applying RPD is related to the higher costs of controlling noise factors than to make the process insensitive to them [28].

Robust optimisation through the RPD concepts of Taguchi may be achieved through different approaches. The Taguchi idea of applying a statistical design to attain a product or process insensitive to environmental disturbances and having the lowest possible cost is considered important in the quality engineering field. However, Taguchi methods are labelled to be inefficient due to the application of saturated designs, interactions neglect, orthogonal arrays inconsistent use for optimisation, the difficulty of interpretation of signal-to-noise ratio and its use as universal criteria for optimisation, and other weaknesses [29]. The Taguchi crossed array designs and SNR metrics are 
yet used but, the ideas and concepts with regard to robustness introduced by Taguchi are nowadays used with more vigorous statistical and optimisation methods.

RPD allied to RSM through combined array approach have been applied effectively, once it enables economy in the experimentation, allows to study the process $\times$ noise factors interactions and let getting mean and variance models for the response through the propagation of error with regard to the noise factors [30]. Mean square error (MSE) can also be applied together with RSM and RPD aiming to approximate the mean of the response to the target and, simultaneously, reduce the variance of the process [31].

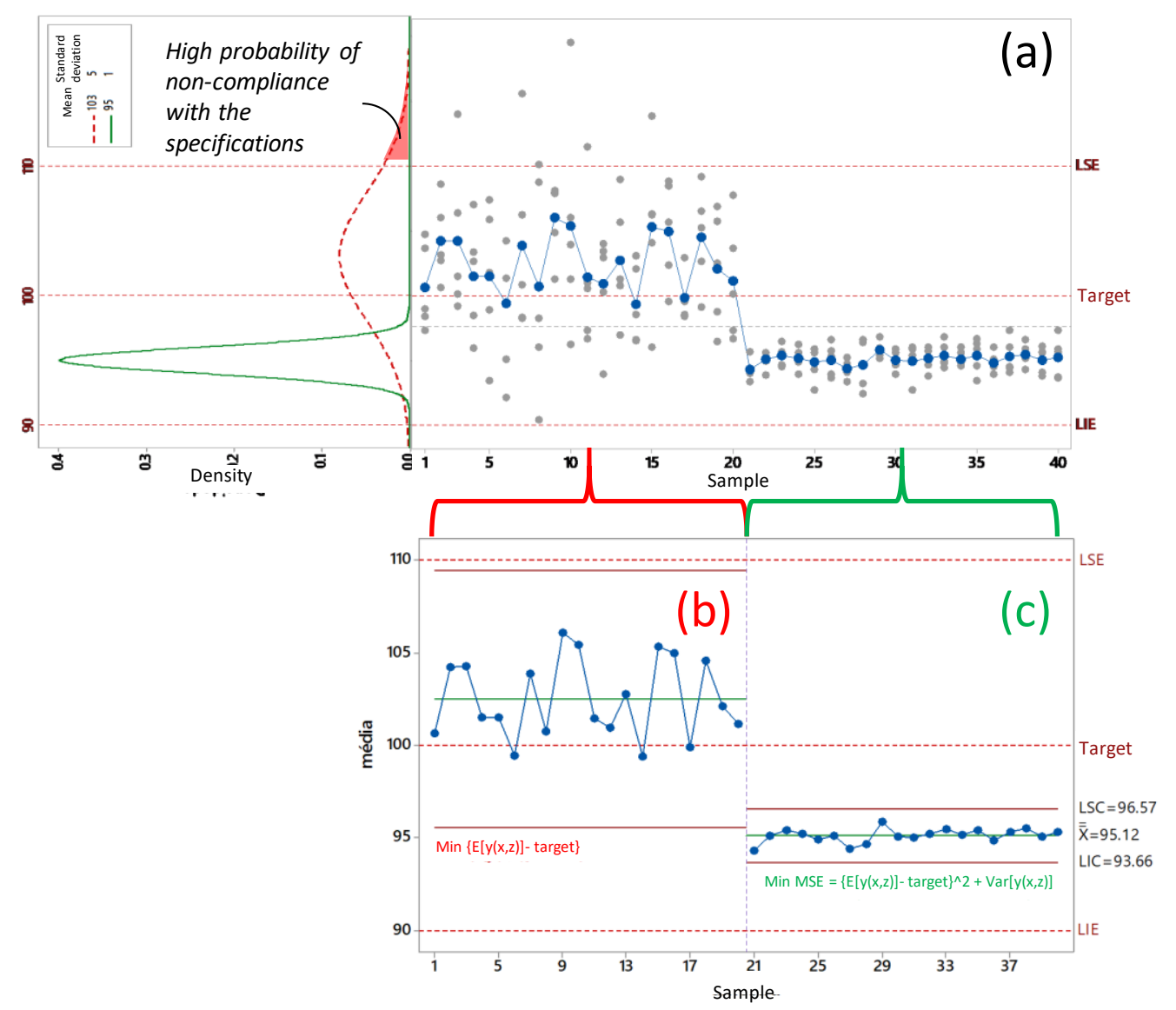

Figure 2. Experiments optimization (a) overview; (b) conventional optimization; (c) robust optimization

Figure 2 highlights the differences between the conventional optimization and the robust optimization. Essentially, in the conventional optimization, Figure 2(b), there is a major concern in approximate the mean from the target defined with regard to the specification limits for the product. However, the level of the mean response closest to the target of the response occasionally may present high variability with regard to the noise factors, resulting in a high probability of non- 
compliance with the specifications. In the robust optimization, Figure 2(c), through the RSM, RPD and MSE approaches deals with the trade-off between mean and variance. Despite in this approach the mean optimal level of the response may remain farther from the target, the robustness may be achieved, diminishing the variance of the response with regard to the noise factors, guaranteeing high possibility of meeting the specifications.

Another important aspect in engineering optimization, apart from the achievement of robustness, is the presence of several responses of interest to be optimized simultaneously. Optimization procedures involving multiple responses are called multi-objective. In this approach, it is impossible to get simultaneously the individual optima for all the evaluated responses. Therefore, the optimal solution is not unique, but it consists of a set of solutions denominated Pareto optimal solutions. A solution is said Pareto optimal when it improves one of the objective functions with the prejudice of almost one of the remaining ones. Throughout a set of Pareto optimal solutions, the region called Pareto front is explored and the trade-off among the objective functions may be studied [32]. There are several multi-optimization approaches available in the literature. The normalized normal constraint method (NNC) [33] presents itself as a good alternative, guaranteeing good exploration of the Pareto frontier and, consequently, the trade-off between the responses.

Helical milling in the soft alloy Al 7075 was addressed considering robust multi-response optimisation [34] and considering multivariate robust optimisation [35]. In both cases the noise factor considered was the tool overhang length. However, the challenges in the helical milling of AISI H13 hardened steels are unique, these are difficult-to-cut materials and presents a significant demand in hole-making. Besides, it is important to take into consideration different noise factors which are critical in the field of hole-making in hardened steel.

Helical milling of AISI H13 hardened steel is challenging due to the high mechanical and wear resistance, inducing tool deflection, geometrical and microgeometrical deviation in helical milling. These problems may be related to the high tool wear rates and high cutting forces developed in helical milling of AISI H13 hardened steel. Consequently, it is important to conjoin different methods to achieve the robust modelling and optimisation of this feasible but challenging operation in a difficult-to-cut material. Then, the combination of RSM, RPD, MSE and NNC methods is justified by the difficulties on hole-making of AISI H13 hardened steel through helical milling."

Since conventional hard drilling presents unfavourable conditions [36], helical milling is a practicable hole-making process which has been applied in these materials with good results $[9,18]$. As AISI H13 hardened steel has been insufficiently studied in helical milling of AISI H13 hardened steel, this study may be a reference for the manufacturers of moulds and dies. To choose suitable helical milling cutting conditions to assure high quality in hole-making in these materials is a 
difficult task. Due to the challenges in the machining of hardened steels, such as AISI H13 hardened, to consider noise factors in the experimental planning is a consistent proposal to attain variability minimization. As important quality parameters of boreholes are expressed with different and, frequently, dissimilar outcomes, such as roughness and roundness, it is important to apply multiobjective optimisation in the helical milling of AISI H13 hardened steel. By conjoining RSM/RPD and multi-objective optimisation, boreholes of AISI H13 hardened steel with robust optimal quality levels of multiple responses may be attained. The same methodology proposed in the present study can be applied in other manufacturing process and systems which present significant noise factors and multiple outcomes of interest as a statistical, mathematical and computational procedure.

The purpose of this investigation is the multi-objective robust modelling and optimization of borehole geometry and roughness in the helical milling of AISI H13 hardened steel. Through the results attained in this study, boreholes with high geometrical and microgeometrical quality with robustness in regard to noise may be achieved in helical milling process of AISI H13 hardened steel. These goals are achieved through the application of the RPD, MSE and NNC methods. This statistical, mathematical and computational approach may also be applied to the robust optimisation of other manufacturing processes.

\section{Helical milling kinematics}

The helical milling kinematics is basically defined by a helical toolpath conjugated with tool rotation around its own axis. The kinematics of helical milling may be studied considering the vector decomposition of the helical feed velocity $\left(v_{f}\right)$ in the tangential and frontal components [14]. This allows the engineer to study the effect of peripheral and frontal cutting in the borehole quality [16]. Figure 3 illustrates the helical milling kinematics. The helical feed velocity $\left(v_{f}\right)$ in $[\mathrm{mm} / \mathrm{min}]$, developed on tool centre point (TCP) can be calculated considering the axial feed velocity of the helix $\left(v_{f h a}\right)$ in $[\mathrm{mm} / \mathrm{min}]$ and the tangential feed velocity of the helix $\left(v_{f h t}\right)$, also in $[\mathrm{mm} / \mathrm{min}]$, according to Equation 1. The axial feed velocity of the helix ( $\left.v_{f h a}\right)$ may be described considering the axial feed per tooth of the helix $\left(f_{z a}\right)$ in [mm/tooth] according to the Equation 2, where $z$ is the number of teeth and $n$ in [rev/min] is the spindle rotation speed. The tangential feed velocity of the helix $\left(v_{f h t}\right)$ in $[\mathrm{mm} / \mathrm{min}]$, may be calculated according to the Equation 3 concerning the tangential feed velocity $\left(v_{f t}\right)$ in $[\mathrm{mm} / \mathrm{min}]$. In the circular cutting, the feed velocity in the periphery of the borehole, $v_{f t}$, related to the borehole diameter $\left(D_{b}\right)$, in $[\mathrm{mm}]$, is higher than the feed velocity in the TCP, $v_{f h}$, related to the helix diameter $\left(D_{h}\right)$ in $[\mathrm{mm}]$. Consequently, the tangential feed per tooth of 
the helix $\left(f_{z t}\right)$ in the tangential direction should be considered in the point of high velocity, $v_{f t}$, according to the Equation 4.

$v_{f}=\sqrt{v_{f h a}^{2}+v_{f h t}^{2}}$

$v_{f h a}=f_{z a} \cdot z \cdot n$

$v_{f h t}=v_{f t} \frac{D_{h}}{D_{b}}$

$v_{f t}=f_{z t} \cdot z \cdot n$

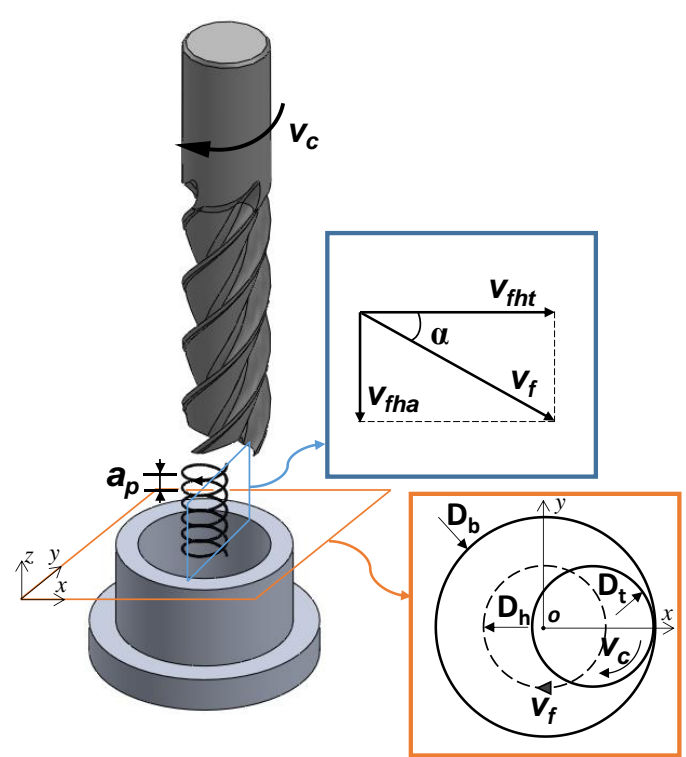

Figure 3. Helical milling kinematics [35] with permission from Springer Nature, license number: 4467180469015

The axial depth of cut $\left(a_{p}\right)$ varies according to the contact angle $(\varphi)$, considering the approach of Denkena et al. [14], or taking an arbitrary inspection ratio, it can be determined the proportions of $a_{p}$ relative to frontal and peripheral cut, according to the approach of Brinksmeier et al. [8].

However, the maximum depth of cut $\left(a_{p}{ }^{*}\right)$ suits as a measure of geometrical mechanical load, since, it is related to chip geometry. The maximum axial depth of cut $\left(a_{p}{ }^{*}\right)$ is mathematically dependent of the feed velocities components in axial and tangential directions [14]. Firstly, the helix angle $(\alpha)$ can be expressed through Equation 5 and, subsequently, $a_{p}{ }^{*}$, which is the helical pitch, in [mm/rev], is calculated through the Equation 6, considering the helix angle and the length of the circular path $\left(\pi \times D_{h}\right)$. By manipulating this expression considering Equations 1-4, $a_{p}{ }^{*}$ may be defined with regard to $f_{z a}$ and $f_{z t}$ to understand the effect of these portions of cut related to axial and peripheral cut, respectively [16]. According to Iyer et al. [37], in helical milling without a pre-hole the radial depth 
of cut $\left(a_{e}\right)$ in $[\mathrm{mm}]$ is measured in the $x y$ plane and is the ratio between the total area removed to the length of the circular path, as expressed in the Equation 7, depending only on the diameters $D_{b}$ and $D_{h}$.

$$
\begin{aligned}
& \alpha=\arctan \left(\frac{v_{f h a}}{v_{f h t}}\right) \\
& a_{p}^{*}=\tan (\alpha) \cdot \pi \cdot D_{h}=\frac{f_{z a} \cdot \pi \cdot D_{b}}{f_{z t}} \\
& a_{e}=\frac{\pi \cdot D_{b}^{2}}{4} \cdot \frac{1}{\pi \cdot D_{h}}=\frac{D_{b}^{2}}{4 \cdot D_{h}}
\end{aligned}
$$

\section{Noise factors identified in the helical milling of hardened steel}

As early stated, a noise factor is a variable which affects the process but cannot be controlled in production level. However, noise factors may be fixed at the experimental stage to achieve process robustness. An important noise factor in mold and die machining is the tool overhang length. Tool overhang length is not defined considering the engineer preferences, but according to the workpiece geometry [35,38]. In cavities machining, it is necessary high tool overhang length to avoid collision between the tool holder and the workpiece. The tool overhang length may originate tool deflection, vibration and, consequently, affect roughness, geometrical error, and productivity $[39,40]$. Hence, it is required to find levels of process parameters which allows robustness with regard to tool overhang length [38].

Workpiece hardness may present variation due to heat treatment, anisotropy, and other metallurgical characteristics. The effect of workpiece hardness was addressed in the helical milling of the AISI 4340 steel [18], nevertheless, it was not studied as a noise factor. The workpiece hardness has been studied as a noise factor in the context of hard machining [41]. Paiva et al. [41] affirmed that, in turning of AISI 52100, after some longitudinal turning passes, the hardness decreased from nearby $50 \mathrm{HRC}$ to approximately $40 \mathrm{HRC}$. Besides the authors affirmed that after heat treatment, the hardness was between 49 and 52 HRC. Then, due to difficulties in heat treatment, the hardness may present significant variation. Since, RPD theory advocates that it is less costly to achieve robustness to difficult-to-control factors than to controll them, in hole-making of hardened materials it is important to achieve control factors levels' guaranteeing insensitive in quality parameters with regard to hardness variation. 
Another specific difficult of hole-making is to achieve a borehole with steady quality in relation to the borehole surface height. In helical milling and other hole-making processes, usually the quality in the borehole end is poorer than in the beginning. It may be related to the high tool-workpiece contact area increasing tool deflection and radial force [14], resulting in dimensional, geometrical and microgeometrical variation [15,42]. Furthermore, in helical milling the tool realizes more orbital revolutions in the beginning than in the end of the borehole, resulting in the smoothing of the surface due to the peripheral cut. Then, the roughness is lower as the borehole height point is closer to the borehole entry [43].

\section{Robust parameter design and mean square error}

RPD aims to find levels of process factors robust with regard to noise factors $[25,44]$. Due to several statistical contradictions of the Taguchi's methods [29], its philosophy was adopted by several researchers in more reliable alternatives to RPD. Some of these proposals highlight the use of RSM procedures to RPD, where mean and variance modelling is achieved [30]. Welch et al. [25] proposed a design including process and noise factors which requires lower runs than the crossed arrays. Shoemaker et al. [26] called this RPD approach combined array, highlighting the importance of process $\times$ noise interactions. Through the study of process $\times$ noise interactions, the propagation of error is feasible and the achievement of levels of process factor robust to noise factors variation is possible.

Box and Jones [31] described a response model in function of process, $\mathbf{x}^{\mathbf{T}}=\left(x_{1}, x_{2}, \ldots, x_{k}\right)$, and noise factors, $\mathbf{z}^{\mathbf{T}}=\left(z_{1}, z_{2}, \ldots, z_{r}\right)$, which may be written in the matrix form according to Equation 8 .

$$
y(\mathbf{x}, \mathbf{z})=\beta_{0}+\mathbf{x}^{\mathbf{T}} \boldsymbol{\beta}+\mathbf{x}^{\mathbf{T}} \mathbf{B} \mathbf{x}+\mathbf{z}^{\mathbf{T}} \boldsymbol{\gamma}+\mathbf{x}^{\mathbf{T}} \Delta \mathbf{z}+\boldsymbol{\varepsilon}
$$

where $\beta_{0}$ is the intercept, $\boldsymbol{\beta}$ is the vector of linear coefficients of process factors, $\mathbf{B}$ is the matrix of second-order coefficients of process factors, containing quadratic and interaction terms, $\gamma$ is the vector of linear coefficients of noise factors and $\Delta$ is the matrix of process $\times$ noise interaction terms [38], as follows: 


$$
\boldsymbol{\beta}_{k \times 1}=\left[\begin{array}{c}
\beta_{1} \\
\vdots \\
\beta_{k}
\end{array}\right] ; \quad \mathbf{B}_{k \times k}=\left[\begin{array}{cccc}
\beta_{11} & \beta_{12} / 2 & \cdots & \beta_{1 k} / 2 \\
& \beta_{22} & \cdots & \beta_{2 k} / 2 \\
& & \ddots & \vdots \\
s y m & & & \beta_{k k}
\end{array}\right] ; \quad \gamma_{r \times 1}=\left[\begin{array}{c}
\gamma_{1} \\
\vdots \\
\gamma_{r}
\end{array}\right] ; \quad \boldsymbol{\Delta}_{k \times r}=\left[\begin{array}{cccc}
\delta_{11} & \delta_{12} & \cdots & \delta_{1 r} \\
\delta_{21} & \delta_{22} & \cdots & \delta_{2 r} \\
\vdots & \vdots & \ddots & \vdots \\
\delta_{k 1} & \delta_{k 2} & \cdots & \delta_{k r}
\end{array}\right]
$$

For the response model in the Equation 8, it is assumed that the experimental error $\varepsilon$ is normally and independently distributed NID $\left(0, \sigma_{\varepsilon}^{2}\right)$. The model takes into account a second-order response surface in function of process factors, $\beta_{0}+\mathbf{x}^{\mathrm{T}} \boldsymbol{\beta}+\mathbf{x}^{\mathrm{T}} \mathbf{B} \mathbf{x}$, linear terms of noise factors, $\mathbf{z}^{\mathrm{T}} \boldsymbol{\gamma}$, in addition to the important process $\times$ noise interactions, $\mathbf{x}^{\mathrm{T}} \boldsymbol{\Delta} \mathbf{z}$. It is presumed that control and noise factors are continuous [46].

Taking into account a coded design with continuous and random noise factors from a vector $\mathbf{z}$, it may be presumed that $E(\mathbf{z})=0$ and $\operatorname{Var}(\mathbf{z})=\mathbf{V}$, where $\mathbf{V}_{r \times r}$, is the variance-covariance matrix of $\mathbf{z}$, symmetrical positive definite, usually assumed diagonal with terms $\sigma_{z j}{ }^{2}=\sigma_{z}^{2}, j=1, \ldots, r$. $\mathbf{V}$ is assumed diagonal since the noise factors are considered not correlated among themselves $[46,47]$.

Applying the mean operator with regard to $\mathbf{z}$ in the Equation 8 [30]:

$$
\underset{\mathbf{z}, \varepsilon}{E}[y(\mathbf{x}, \mathbf{z})]=\hat{\beta}_{0}+\mathbf{x}^{\mathrm{T}} \hat{\boldsymbol{\beta}}+\mathbf{x}^{\mathrm{T}} \hat{\mathbf{B}} \mathbf{x}
$$

Analogously, the variance model is obtained through the application of the variance operator with respect to $\mathbf{z}$ in the Equation 8 [30].

$$
\operatorname{Var}[y(\mathbf{x}, \mathbf{z})]=\left(\hat{\gamma}+\hat{\boldsymbol{\Delta}}^{\mathbf{T}} \mathbf{x}\right)^{\mathbf{T}} \mathbf{V}\left(\hat{\gamma}+\hat{\boldsymbol{\Delta}}^{\mathbf{T}} \mathbf{x}\right)+\hat{\sigma}_{\varepsilon}^{2}
$$

The slope in the direction of noise factors is important to predict the process variance of the response in the experimental region. The slope is the vector of partial derivatives of the response model in the Equation 8 with respect to $\mathbf{z}[30,46,47]$, according to the Equation 11.

$$
\hat{\mathbf{I}}(\mathbf{x})=\frac{\partial \hat{y}}{\partial \mathbf{z}}=\hat{\gamma}+\hat{\Delta}^{\mathrm{T}} \mathbf{x}
$$

The variance-covariance matrix $\mathbf{V}$ is assumed diagonal and it may be presumed that $\sigma_{z j}{ }^{2}=\sigma_{z}{ }^{2}=$ 1 , due to the codification of the noise factors levels in \pm 1 , in a way that the variance-covariance 
matrix is assumed as an identity matrix, $\mathbf{V}=\mathbf{I}$. Consequently, the Equation 10 may be rewritten as [48]:

$\operatorname{Var}_{\mathbf{z}, \varepsilon}[y(\mathbf{x}, \mathbf{z})]=\left(\hat{\gamma}+\hat{\boldsymbol{\Delta}}^{\mathbf{T}} \mathbf{x}\right)^{\mathbf{T}}\left(\hat{\gamma}+\hat{\boldsymbol{\Delta}}^{\mathbf{T}} \mathbf{x}\right)+\hat{\sigma}_{\varepsilon}^{2}=\hat{\mathbf{l}}^{\mathbf{T}} \hat{\mathbf{l}}+\hat{\sigma}_{\varepsilon}^{2}$

Figure 4 presents two graphical examples of process $\times$ noise interactions. In the first case, Figure 4(a), it is clear that the effect of $x_{1}$ depends on the level of $z_{1}$. However, the most important aspect in RPD is that it is possible to achieve a level of $x_{1}$ which makes $y$ insensitive to the variation of $z_{1}$. Them, setting $x_{1}=0.5$ makes $y$ robust to the variation of $z_{1}$. In the case of the interaction between $x_{1}$ and $z_{2}$ it is clear that it is not possible to achieve a level of $x_{1}$ which makes $y$ robust to $z_{2}$ variation since in all the domain of $x_{1}$ the effect of $z_{2}$ is significant in the response. Consequently, when the process $\times$ noise interaction is not significant it is not feasible to achieve robustness concerning noise variation.
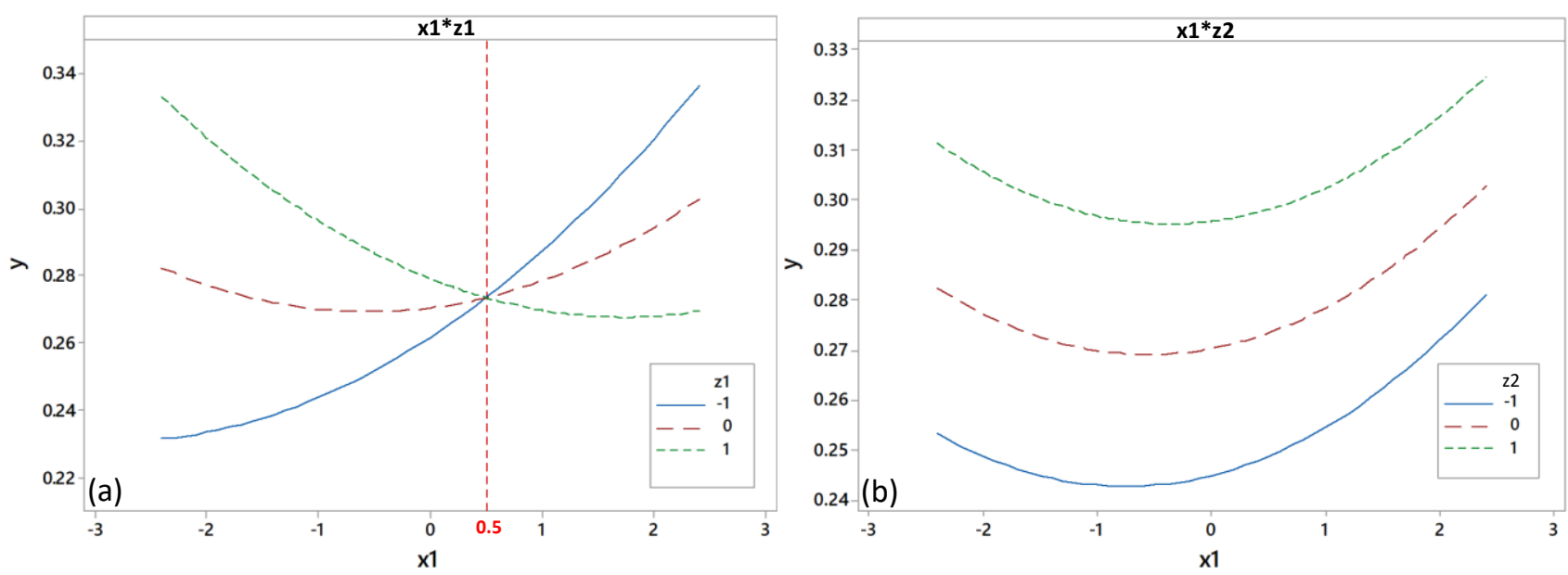

Figure 4. Process $\times$ noise (a) significant interaction; (b) non-significant interaction

Taking the response model in the Equation 8, in a general form, to facilitate the understanding of the least squares estimates for the response model, it may be written [47]:

$\mathbf{y}(\mathbf{x}, \mathbf{z})=\mathbf{X}^{*} \boldsymbol{\beta}^{*}+\mathbf{\varepsilon}$

The matrix $\mathbf{X}^{*}$ presents $1+2 k+k(k-1)+r+r k$ columns taking into account the intercept, the linear, second order interactions, and quadratic effects of the process factors, the linear effects of the noise factors, and the process $\times$ noise interactions, respectively. Similarly, $\boldsymbol{\beta}^{*}$ comprises the 
response model coefficients $\beta_{0}, \boldsymbol{\beta}, \mathbf{B}, \boldsymbol{\gamma}$, and $\Delta$. The least squares approximation for $\boldsymbol{\beta}^{*}$ is found in the Equation 14 [47].

$$
\boldsymbol{\beta}^{*}=\left(\mathbf{X}^{* \mathrm{~T}} \mathbf{X}^{*}\right)^{-1} \mathbf{X}^{*} \mathbf{y}
$$

The matrix $\mathbf{C}^{*}=\left(\mathbf{X}^{* \mathrm{~T}} \mathbf{X}^{*}\right)^{-1}$ is composed by important submatrices, as illustrated in Figure 5 . The submatrix $\mathbf{C}^{* k l}, k, l=1,2,3$, are the variance-covariance matrices for various subsets of factors of the response model, as illustrated in Figure 5 [47].

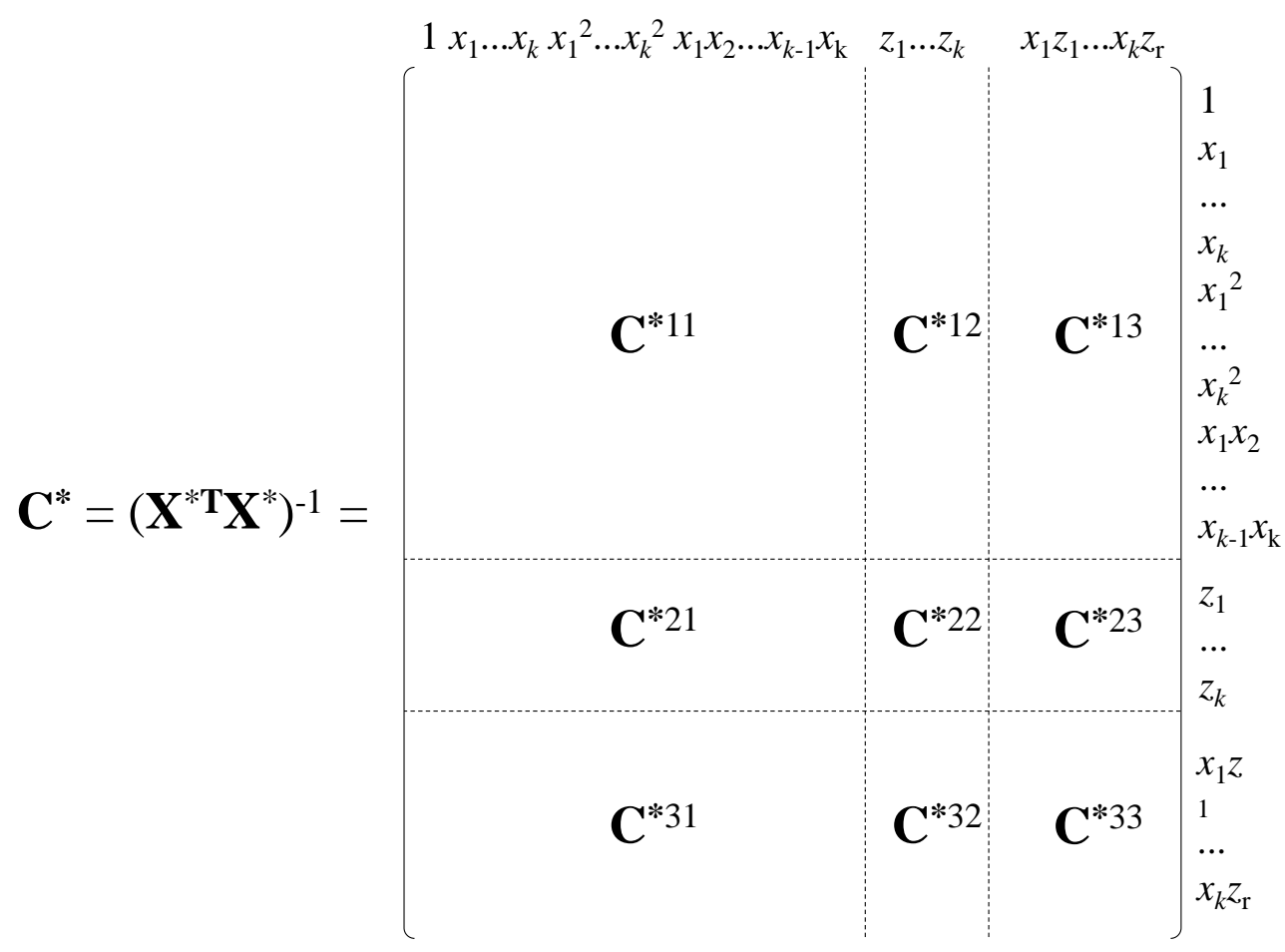

Figure 5. Structure of the submatrix of $\mathbf{C}^{*}$ (adapted from BORROR et al. [47])

When using RPD combined array approach, the guarantee of homoscedasticity may not be confirmed due to the presence of the difficult-to-control noise factors. In these cases, the response model achieved by ordinary least squares (OLS) may present prediction problems. In these cases, the weighted least squares (WLS) may be employed.

The matrix $\mathbf{C}^{*}$ may be considered to WLS estimates, standing as $\mathbf{C}^{*}=\left(\mathbf{X}^{* \mathrm{~T}} \mathbf{W}^{*} \mathbf{X}^{*}\right)^{-1}$, and the coefficients obtained by the WLS are $\boldsymbol{\beta}^{*}=\left(\mathbf{X}^{* \mathrm{~T}} \mathbf{W}^{*} \mathbf{X}^{*}\right)^{-1} \mathbf{X}^{*} \mathbf{W}^{*} \mathbf{y}$, where $\mathbf{W}^{*}$ is a diagonal matrix containing the weights related to the heteroscedastic variance of the observations. The OLS method presents the assumption $\operatorname{Cov}(\boldsymbol{\varepsilon})=\sigma^{2} \mathbf{I}$, which implies in the independence of $y_{i}$ and homocedasticity, 
i.e., variance homogeneity, $\sigma^{2}, i=1, \ldots, N$, which is not easy to achieve in RPD. In the presence of heteroscedasticity, the model $\mathbf{y}(\mathbf{x}, \mathbf{z})=\mathbf{X}^{*} \boldsymbol{\beta}^{*}+\boldsymbol{\varepsilon}$ presents $\boldsymbol{\varepsilon} \sim \mathrm{N}\left(0, \sigma^{2} \mathbf{W}\right)[49,50]$. Romano and Wolf [51] showed that WLS is more efficient than OLS also with nonnormal error terms.

The use of a weighted residual sum of squares is remedial when some observations present more prominent error than others. Consequently, observations with lower variances will receive larger weight in the WLS regression. Then, instead of considering the variance constant for each observation, it can be considered that $\operatorname{Var}\left(y_{i}\right)=\sigma^{2} / w_{i}$. The variance function is yet constant, however the individual variance $i=1, \ldots, N$ differs for each observation [50]. In the appendix $\mathrm{A}$ it is demonstrated that OLS results may be applied to WLS problems.

Mean square error optimization is commonly used together with RPD to allow bias and variance optimization. The mean square error measures the expectancy of the quadratic deviations of an estimator. Then, the MSE is a measure of approximation of the estimated value with regard to a target value. Box and Jones [31] proposed the first application of MSE in RPD. Vining and Myers [52] affirmed that this application is more recommended with combined array approach. Lin and Tu [53] proposed the same formulation of Box and Jones [31], denoting this approach as dual optimization. This formulation with regard to mean and variance Equations 9 and 12, is defined in Equation 15 , where $T_{y}$ is the target value for $\underset{\mathbf{z}, \mathbf{\varepsilon}}{E}[y(\mathbf{x}, \mathbf{z})]$ obtained through $T_{y}=\underset{\mathbf{x} \in \Omega}{\operatorname{Min}}\{\underset{\mathbf{z}, \mathbf{z}}{E}[y(\mathbf{x}, \mathbf{z})]\}$.

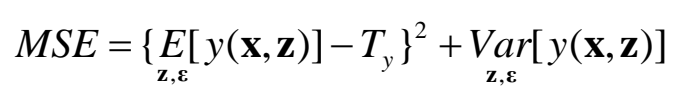

\section{Similarity study through cluster analysis}

The cluster analysis aims to group similar objects in the same group and the dissimilar ones in different groups. The analysis may be applied to group observations or variables. For $m$ variables to be hierarchically grouped the analysis begging with $m$ groups, each one with one variable and finishes with a group with all variables. In each step, the variables are grouped throughout an adopted similarity measure. Consider the data of the $m$ variables in the matrix $\mathbf{Y}$ as follows [54]. 


$$
\mathbf{Y}=\left[\begin{array}{cccccc}
y_{11} & y_{12} & \cdots & y_{1 j} & \cdots & y_{1 m} \\
y_{21} & y_{22} & \cdots & y_{2 j} & \cdots & y_{2 m} \\
\vdots & \vdots & & \vdots & & \vdots \\
y_{i 1} & y_{i 2} & \cdots & y_{i j} & \cdots & y_{i m} \\
\vdots & \vdots & & \vdots & & \vdots \\
y_{N 1} & y_{N 2} & \cdots & y_{N j} & \cdots & y_{N m}
\end{array}\right]
$$

A proximity matrix is necessary to start the analysis, storing the similarity or dissimilarity among the variables. For instance, the correlation is a similarity measure, while the distance is a dissimilarity one. The proximity matrix $\mathbf{P}$ of order $m \times m$ is a matrix of proximity coefficients $p_{r s}$ between two variables $y_{r}$ and $y_{s}$, with $m(m-1)$ proximity measures results, as follows. The matrix $\mathbf{P}$ is symmetrical, i.e., $p_{r s}=p_{s r}[54]$.

$$
\mathbf{P}=\left[\begin{array}{cccc}
p_{11} & p_{12} & \cdots & p_{1 m} \\
p_{21} & p_{22} & \cdots & p_{2 m} \\
\vdots & \vdots & \ddots & \vdots \\
p_{m 1} & p_{m 2} & \cdots & p_{m m}
\end{array}\right]
$$

An example of similarity measure is the quadratic measure between two variables $\mathbf{y}_{r}$ and $\mathbf{y}_{s}$ as follows [54]:

$$
d_{r s}^{2}=\left(\mathbf{y}_{r}-\mathbf{y}_{s}\right)^{\mathbf{T}} \boldsymbol{\Psi}\left(\mathbf{y}_{r}-\mathbf{y}_{s}\right)
$$

with $\boldsymbol{\Psi}$ as a metric of interest. When $\boldsymbol{\Psi}=\mathbf{I}$, being $\mathbf{I}$ and identity matrix, this quadratic distance is the Euclidian distance, which is recommended for the cases when the variables present similar scales. When $\Psi=\operatorname{diag}\left(1 / s_{i i}\right)$, with $i=1, \ldots, \mathrm{N}$ and $s_{i i}$ the variance of $i$-th variable, $d_{r s}{ }^{2}$ is the quadratic Euclidian distance, being this measure suitable for non-correlated variables with distinct scales. To consider the correlation, it is recommended to use $\boldsymbol{\Psi}=\mathbf{s}^{-1}$, with $\mathbf{s}$ as the sampling variancecovariance matrix, with $d_{r s}{ }^{2}$ in this case as the generalized Mahalanobis distance [54].

Taking the vectors of values of the variables $\mathbf{y}_{r}$ e $\mathbf{y}_{s}$ in the $N$-dimensional space of the variables, a similarity measure $s_{r s}=s_{s r}$ presents domain $[0 ; 1]$. If $s_{r s}=1$, the variables present perfect similarity, it is, $\mathbf{y}_{r}=\mathbf{y}_{s}$. The dissimilarity can be obtained considering the similarity through $d_{r s}=1-$ $s_{r s}$. However, the contrary is not possible, since distance measures present domain $[0 ; \infty)$. The correlation coefficient $r_{r s}$ is used as controversial similarity measure, since its domain is $[-1 ; 1]$ and, 
when $r_{r s}=1$, it does not mean that the similarity is perfect, but that there is a perfect linear relationship between the variables. It is suggested to use the absolute correlation $\left|r_{r s}\right|$ as a similarity measure, however, the problem of the perfect similarity persists. When clustering variables, it is generally used $\left|r_{r s}\right|$ as a similarity measure and $d_{r s}=1-\left|r_{r s}\right|$ as dissimilarity measure [54].

The hierarchical grouping methods classify the variables in sequential steps, representing the groups using a dendrogram. A general algorithm for $m$ variables may be described as follows [54]:

a. To obtain the proximity matrix considering the similarities or dissimilarities of order $m \times m$ between the variables. In the Ward method the proximity measure is $\left[p_{i j}\right]=$ $\left[0,5 d_{i j}^{2}\right]$;

b. To start the analysis with $m$ groups each with one variable;

c. To identify in the matrix $\mathbf{P}$ the pair with the highest proximity and represent the distance between them by $p_{r s}$;

d. To merge the groups $r$ and $s$ forming a new group $r s$. Recalculate the matrix $\mathbf{P}$ eliminating the lines and columns $r$ and $s$ and creating a new line and column $r s$. In the Ward method, the calculation of the distance between thr group $r s$ and other group $t$ is calculated as in Equation 19, with $m_{t}$ and $m_{r s}$ as the number of variables in the groups $r s$ and $t$ respectively.

$$
p_{(r s) t}=\frac{1}{m_{t}+m_{r s}}\left[\left(n_{t}+n_{r}\right) p_{r t}+\left(n_{t}+n_{s}\right) p_{s t}-n_{t} p_{r s}\right]
$$

e. To repeat the steps $c$ to $d$ until grouping all the variables hierarchically.

\section{Normalized normal constraint multi-objective optimization method}

A multi-objective optimization task is related with the simultaneous optimization of $m$ objective functions. In these problems, the solution is not unique, but a set of solutions called Pareto optimal or non-inferior solutions. Operationally, the performance of some of the $m$ objective functions cannot be improved without the worsening of at least one of the $m-1$ remaining one [32]. Multi-objective tasks are frequently employed to solve engineering problems. Recently, multiobjective optimization was applied on steel case hardening to solve the trade-off between hardness and residual stresses [55]. The trade-off between minimum weldline temperature and clamping force in plastic injection moulding was also solved through multi-objective optimization [56]. 
The terminology of multi-objective optimization should be presented. A generic multiobjective optimization problem is defined as follows:

$$
\operatorname{Min}_{(\mathbf{x} \in \Omega)} \mathbf{f}(\mathbf{x})=\left[f_{i}(\mathbf{x}), \ldots, f_{m}(\mathbf{x})\right]
$$

were $f_{i}$ represents the $i$-th objective function $i=1, \ldots m, \mathbf{x}=\left[x_{1}, \ldots, x_{k}\right]^{\mathrm{T}}$ is the vector of control factors, $\Omega$ is the experimental space and $\mathbf{f}(\mathbf{x})=\left[f_{1}(\mathbf{x}), \ldots, f_{m}(\mathbf{x})\right]^{\mathrm{T}}$ is the vector of objective functions. The anchor points are the individual minima for each objective function $\mathbf{f}_{1}{ }^{*}, \ldots, \mathbf{f}_{\mathrm{m}}{ }^{*}, i=1, \ldots, m$, resulting in an optimal vector $\mathbf{x}_{i}{ }^{*}$. The anchor point related to the $i$-th objective function is $\left[f_{l}\left(\mathbf{x}_{i}{ }^{*}\right), \ldots\right.$, $\left.f_{i}^{*}\left(\mathbf{x}_{i}{ }^{*}\right), \ldots, f_{m}\left(\mathbf{x}_{i}{ }^{*}\right)\right]^{\mathrm{T}}$. The utopia point is the vector $\mathbf{f}^{\mathrm{U}}=\left[f_{1}{ }^{*}, \ldots, f_{m}{ }^{*}\right]^{\mathrm{T}}$ or explicitly $\mathbf{f}^{\mathrm{U}}=\left[f_{1}\left(\mathbf{x}_{1}{ }^{*}\right), \ldots\right.$, $\left.f_{m}\left(\mathbf{x}_{m}{ }^{*}\right)\right]^{\mathrm{T}}$, i.e., $f_{i}^{*}=f_{i}\left(x_{i}{ }^{*}\right)$. The utopia hyperplane (for $m=2$, the utopia line and $m=3$ utopia plane) is the hyperplane which connects all the anchor points.

The nadir point $\mathbf{f}^{\mathrm{N}}=\left[f_{1}{ }^{N}, \ldots, f_{m}{ }^{N}\right]^{\mathrm{T}}$, is the combination of the maximization of all objective functions individually, i.e., $\mathbf{f}^{\mathrm{N}}=\left[\operatorname{Max}_{(x \in \Omega)} f_{i}(\mathbf{x}), \ldots, \operatorname{Max}_{(x \in \Omega)} f_{m}(\mathbf{x})\right]^{\mathrm{T}}$, is, therefore, the combination of the worst results of the objective functions. The nadir and utopia points are not in the feasible region. The pseudo-nadir point is the combination of the worst result of each objective function considering the application of the optimal vector $\mathbf{x}_{i}{ }^{*}$ obtained in the optimisation of the other functions, defined as $\mathbf{f}^{\mathrm{PN}}=\left[f_{1}^{P N}, \ldots, f_{m}^{P N}\right]^{\mathrm{T}}$. The pseudo-nadir point may be inside or outside the feasible region, depending on the objective functions. For the $i$-th objective function, the pseudo-nadir point is defined as $f_{i}^{P N}=\operatorname{Max}_{(x \in \Omega)}\left\{f_{i}\left(\mathbf{x}_{1}^{*}\right), \ldots, f_{i}\left(\mathbf{x}_{m}^{*}\right)\right\}, i=1, \ldots, m$.

To avoid scale and size effect, it is important to normalize the objective functions and the objective space. In the normalized solution space, the utopia and pseudo-nadir points are $\overline{\mathbf{f}}^{\mathrm{U}}=[0, \ldots$, $0]^{\mathrm{T}}$ and $\overline{\mathbf{f}}^{\mathrm{PN}}=[1, \ldots, 1]^{\mathrm{T}}$, respectively. The normalization is defined according to the Equation 21 . The normalized vector of objective functions is $\overline{\mathbf{f}}=\left[\bar{f}_{1}, \ldots, \bar{f}_{m}\right]^{T}$.

$$
\bar{f}_{i}=\frac{f_{i}-f_{i}^{*}}{f_{i}^{P N}-f_{i}^{*}}
$$

The payoff matrix measures the trade-off limits among the $m$ objective functions. The main diagonal is composed of the individual minima of the objective functions which composes the utopia vector $\mathbf{f}^{\mathrm{U}}$. The pseudo-nadir vector is achieved as the maximization of the values of the payoff matrix 
rows. Besides, each column of the payoff matrix is an anchor point. The payoff matrix is exposed in the Equation 22 and the normalized payoff matrix is exposed in the Equation 23.

$$
\boldsymbol{\Phi}=\left[\begin{array}{ccccc}
f_{1}^{*}\left(\mathbf{x}_{1}^{*}\right) & \cdots & f_{1}\left(\mathbf{x}_{i}^{*}\right) & \cdots & f_{1}\left(\mathbf{x}_{m}^{*}\right) \\
\vdots & \ddots & \vdots & & \vdots \\
f_{i}\left(\mathbf{x}_{1}^{*}\right) & \cdots & f_{i}^{*}\left(\mathbf{x}_{i}^{*}\right) & \cdots & f_{i}\left(\mathbf{x}_{m}^{*}\right) \\
\vdots & & \vdots & \ddots & \vdots \\
f_{m}\left(\mathbf{x}_{1}^{*}\right) & \cdots & f_{m}\left(\mathbf{x}_{i}^{*}\right) & \cdots & f_{m}^{*}\left(\mathbf{x}_{m}^{*}\right)
\end{array}\right]
$$

$$
\overline{\mathbf{\Phi}}=\left[\begin{array}{ccccc}
\frac{f_{1}^{*}\left(\mathbf{x}_{1}^{*}\right)-f_{1}^{*}}{f_{1}^{P N}-f_{1}^{*}} & \cdots & \frac{f_{1}\left(\mathbf{x}_{i}^{*}\right)-f_{1}^{*}}{f_{1}^{P N}-f_{1}^{*}} & \cdots & \frac{f_{1}\left(\mathbf{x}_{m}^{*}\right)-f_{1}^{*}}{f_{1}^{P N}-f_{1}^{*}} \\
\vdots & \ddots & \vdots & & \vdots \\
\frac{f_{i}\left(\mathbf{x}_{1}^{*}\right)-f_{i}^{*}}{f_{i}^{P N}-f_{i}^{*}} & \cdots & \frac{f_{i}^{*}\left(\mathbf{x}_{i}^{*}\right)-f_{i}^{*}}{f_{i}^{P N}-f_{i}^{*}} & \cdots & \frac{f_{i}\left(\mathbf{x}_{m}^{*}\right)-f_{i}^{*}}{f_{i}^{P N}-f_{i}^{*}} \\
\vdots & & \vdots & \ddots & \vdots \\
\frac{f_{m}\left(\mathbf{x}_{1}^{*}\right)-f_{m}^{*}}{f_{m}^{P N}-f_{m}^{*}} & \cdots & \frac{f_{m}\left(\mathbf{x}_{i}^{*}\right)-f_{m}^{*}}{f_{m}^{P N}-f_{m}^{*}} & \cdots & \frac{f_{m}^{*}\left(\mathbf{x}_{m}^{*}\right)-f_{m}^{*}}{f_{m}^{P N}-f_{m}^{*}}
\end{array}\right]
$$

The normalized normal constraint method (NNC) [29] may be applied to achieve a set of Pareto optimal points. The NNC formulation is expressed as follows and may be solved to achieve each Pareto optimal solution with regard to a point $\overline{\mathbf{Q}}_{\mathrm{ij}}, j=1, \ldots, n_{s u b}$, in the utopia line.

$$
\operatorname{Min}_{(\mathbf{x} \in \Omega)}\left\{\bar{f}_{m}\right\}
$$

Subject to:

$$
\overline{\mathbf{N}}_{\mathbf{r}}^{\mathbf{T}}\left(\overline{\mathbf{f}}-\overline{\mathbf{Q}}_{\mathrm{ij}}\right) \leq 0
$$




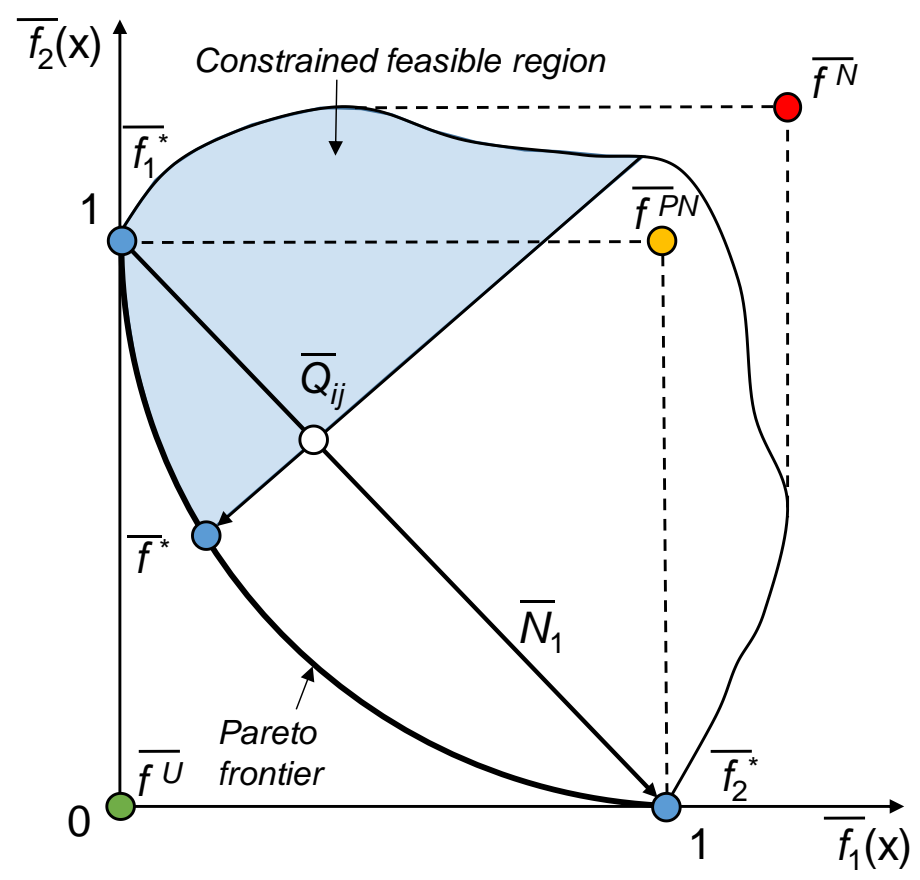

Figure 6. Normalized normal constraint method. Adapted from Pereira et al. [34], with permission from Elsevier, license number: 4467180839435

The formulation in Equations 24 and 25 may be illustrated in the normalized solution space according to Figure 6 for the bi-objective case. In the Equation 24 the $m$-th normalized objective function, $i=1, \ldots, m$, should be minimized. The constraint in Equation 25 is the normalized normal constraint, which is the product between the utopia line vector $\overline{\mathbf{N}}_{\mathrm{r}}, \overline{\mathbf{N}}_{\mathrm{r}}=\overline{\mathbf{f}}_{\mathrm{m}}^{*}-\overline{\mathbf{f}}_{\mathrm{r}}^{*}, r=1, \ldots, m-1$, and the vector $\left(\overline{\mathbf{f}}-\overline{\mathbf{Q}}_{\mathrm{ij}}\right)$ from the point $\overline{\mathbf{Q}}_{\mathrm{ij}}$ to the searched Pareto optimal solution $\overline{\mathbf{f}}$ which will be denoted $\overline{\mathbf{f}}^{*}$ after optimization. As the "normal" constraint is an inequality, the vector $\left(\overline{\mathbf{f}}-\overline{\mathbf{Q}}_{\mathrm{ij}}\right)$ is quasi-normal to the utopia line vector $\overline{\mathbf{N}}_{\mathrm{r}}$, forcing the non-linear optimization method to achieve the minima in the constrained space, giving the vector the possibility of deflecting of dominated regions. Through NNC, a set of well-distributed Pareto optimal solutions may be found in the Pareto frontier.

To achieve a set of solutions in the Pareto frontier, the point $\overline{\mathbf{Q}}_{\mathrm{ij}}$ should be modified considering the weights $w_{i j}$, attributed to the objective functions, $i=1, \ldots, m$, according to Equations 26 to 28 .

$\overline{\mathbf{Q}}_{\mathrm{ij}}=\sum_{\mathrm{i}=1}^{\mathrm{m}} w_{i j} \cdot \overline{\mathbf{f}}_{\mathrm{i}}^{*}$

$0 \leq w_{i j} \leq 1$ 
$\sum_{i=1}^{m} w_{i j}=1$

To solve the NNC method considering a set of weights' vector, $j=1, \ldots, n_{s u b}$, the number of subproblems $\left(n_{\text {sub }}\right)$ is calculated according to the Equation 29, considering the desired spacing $\delta_{r}$ among the points in the utopia line vector $\overline{\mathbf{N}}_{\mathrm{r}}$, where $\eta_{r}=1+1 / \delta_{r}$ is the number of points in the utopia line vector. For the bi-objective case $n_{s u b}=\eta_{r}$.

$n_{\text {sub }}=\left(\begin{array}{c}m+\eta_{r}-2 \\ \eta_{r}-1\end{array}\right)$

The NNC method can be formulated in algebraic notation to facilitate its understanding and implementation [57]. Considering $m=2$ objective functions, $r=m-1=1$. Consequently, the utopia line vector $\overline{\mathbf{N}}_{1}$ in the constraint in Equation 25 may be calculated as follows:

$$
\begin{aligned}
& \bar{N}_{1}=\bar{f}_{2}^{*}-\bar{f}_{1}^{*} \\
& \bar{N}_{1}=\left[\begin{array}{l}
1 \\
0
\end{array}\right]-\left[\begin{array}{l}
0 \\
1
\end{array}\right] \\
& \bar{N}_{1}=\left[\begin{array}{c}
1 \\
-1
\end{array}\right]
\end{aligned}
$$

The vector that links the point $\overline{\mathbf{Q}}_{\mathrm{ij}}$ to the sought Pareto optimal solution, $\bar{f}-\bar{Q}_{i j}$, for $m=2$, is derived as follows:

$$
\begin{aligned}
\bar{f}-\bar{Q}_{i j} & =\left[\begin{array}{l}
\bar{f}_{1} \\
\bar{f}_{2}
\end{array}\right]-\sum_{i=1}^{2} w_{i j} \cdot \bar{f}_{i}^{*}=\left[\begin{array}{l}
\bar{f}_{1} \\
\bar{f}_{2}
\end{array}\right]-\left(w_{1 j} \times \bar{f}_{1}^{*}+w_{2 j} \times \bar{f}_{2}^{*}\right) \\
\bar{f}-\bar{Q}_{i j} & =\left[\begin{array}{l}
\bar{f}_{1} \\
\bar{f}_{2}
\end{array}\right]-\left(w_{1 j}\left[\begin{array}{l}
0 \\
1
\end{array}\right]+w_{2 j}\left[\begin{array}{l}
1 \\
0
\end{array}\right]\right)=\left[\begin{array}{l}
\bar{f}_{1} \\
\bar{f}_{2}
\end{array}\right]-\left[\begin{array}{l}
0+w_{2 j} \\
w_{1 j}+0
\end{array}\right] \\
\bar{f}-\bar{Q}_{i j} & =\left[\begin{array}{l}
\bar{f}_{1}-w_{2 j} \\
\bar{f}_{2}-w_{1 j}
\end{array}\right]
\end{aligned}
$$

Consequently, by using the Equations 30 and 31, the constraint in Equation 25 may be expressed as follows: 
$\bar{N}_{1}^{T}\left(\bar{f}-\bar{Q}_{i j}\right) \leq 0$

$\left[\begin{array}{ll}1 & -1\end{array}\right]\left[\begin{array}{c}\bar{f}_{1}-w_{2 j} \\ \bar{f}_{2}-w_{1 j}\end{array}\right] \leq 0$

$\left(\bar{f}_{1}-w_{2 j}\right)-\left(\bar{f}_{2}-w_{1 j}\right) \leq 0$

$\bar{f}_{1}-\bar{f}_{2}+w_{1 j}-w_{2 j} \leq 0$

In this way, the NNC method for $m=2$ objective functions can be explicitly formulated as follows:

$\underset{(\mathbf{x} \in \Omega)}{\operatorname{Min}}\left\{\bar{f}_{2}\right\}$

Subject to:

$$
\bar{f}_{1}-\bar{f}_{2}+w_{1 j}-w_{2 j} \leq 0
$$

After solving the NNC method formulated in Equations 24 and 25 or explicitly through Equations 33 and 34 , for $j=1, \ldots, n_{s u b}$, it is necessary to apply a Pareto filter. The Pareto filter compares each solution to all remaining solutions obtained in the frontier. It is well-known that a solution is Pareto optimal if it improves at least one of the $m$ objective functions in the multiobjective optimisation. Taking two solutions $\overline{\mathbf{f}}_{p}^{*}$ and $\overline{\mathbf{f}}_{q}^{*}$, if $\bar{f}_{1 p}<\bar{f}_{1 q}, \bar{f}_{2 p}<\bar{f}_{2 q}, \ldots, \bar{f}_{m p}<\bar{f}_{m q}$, the solution $\overline{\mathbf{f}}_{q}^{*}$ is dominated by the solution $\overline{\mathbf{f}}_{p}^{*}$. On the contrary, if $\bar{f}_{1 p}<\bar{f}_{1 q}, \ldots, \bar{f}_{i p}>\bar{f}_{i q}, \ldots, \bar{f}_{m p}<\bar{f}_{m q}$, i.e., the point $\overline{\mathbf{f}}_{q}^{*}$ improves at least one objective function, the two solutions are considered local Pareto optimal with regard to each other. The solution is considered global Pareto optimal only if after the Pareto filtering process, it presents the behaviour of this last case in all comparisons.

NNC is a priori multi-objective method. In a priori approaches the decision maker needs to state its preference prior to the optimisation, for example, defining weights for each objective function [58]. Other a priori multi-objective optimization methods are the well-known weighted sum (WS) and the normal boundary intersection (NBI) [59]. Das and Dennis [60] showed that the WS method fails on achieving an even spread Pareto set in the frontier not only in non-convex Pareto fronts but also in convex Pareto fronts an even spread of weights does not produce an even spread of Pareto optimal solutions. Then, it was presented the NBI method aiming to achieve an evenly distributed Pareto optimal solutions [59]. The formulation of the bi-objective NBI method is similar to the formulation in Equations 33 and 34, however, in NBI the constraint in Equation 34 is an equality instead of an inequality [57]. 
The distribution of the points in the Pareto frontier is very important to guarantee that the weights express the desired preference for the objective functions in evaluation. A measure of the distributions evenness of the Pareto points was proposed and may be useful to quantify the distribution of the Pareto optimal solutions achieved [61]. Messac and Mattson [61] proposed as a measure of evenness the coefficient of variation of the two distances between each Pareto solution and the adjacent Pareto points. However, considering each Pareto point as to calculate the distances, $i$ $=1, \ldots, n_{\text {sub }}$, the distances are duplicated since the distance from $\overline{\mathbf{f}}_{p}^{*}$ to $\overline{\mathbf{f}}_{q}^{*}$ is the same as the distance from $\overline{\mathbf{f}}_{q}^{*}$ to $\overline{\mathbf{f}}_{p}^{*}$. Here is proposed to adopt another similar measure, by considering the coefficient of variation of the vector of Euclidian distances between the adjacent Pareto optimal solutions, calculated in the normalised solutions space, $\bar{d}_{j}=1, \ldots, n_{\text {sub }}-1$. For $n_{\text {sub }}$ solutions, there will be $n_{\text {sub }}$ -1 adjacent distances $\bar{d}_{j}, j=1, \ldots, n_{s u b}-1$, with $\overline{\mathbf{d}}$ as the vector of distances. The coefficient of variation of the Euclidian distance between the adjacent Pareto optimal solutions is calculated as the ratio between the sample standard deviation and the mean of $\overline{\mathbf{d}}$, i.e., $C V_{\overline{\mathbf{d}}}=\sigma_{\overline{\mathbf{d}}} / \mu_{\overline{\mathbf{d}}}$. If $C V_{\overline{\mathbf{d}}}=0$, then the Pareto optimal solutions are perfectly evenly distributed.

Messac et al. [33] presented the NNC method aiming to deal with some drawbacks of NBI method, such as the scale effect since NBI is formulated in the original scale of the objective functions. Besides, the authors presented the notion of Pareto filtering to achieve only a set of Pareto points considering the obtained solutions in the frontier, while this concept is neglected in the NBI proposal. The NNC method is frequently more efficient than the NBI method due to the inequality constraint which is more flexible in deviating of dominated regions of the Pareto frontier.

For $m \geq 3$ objective functions, the exploitation of the Pareto frontier in the NNC and NBI methods, is not complete, as a consequence of the definition of the anchor points considering the pseudo-nadir points. The constraint may not achieve the solutions out of the region delimitated by the utopia line vectors $\overline{\mathbf{N}}_{\mathrm{r}}[61,62]$. To deal with this limitation in the NNC method Messac and Mattson [61] proposed to relax the weights limits in the constraint exposed in Equation 27. Another approach to deal with this problem was proposed by Sanchis et al. [62] by transforming the payoff matrix, which is composed by the anchor points, to achieve its ideal form to guarantee a better exploitation of the Pareto front. The limitation of the anchor points and, consequently, of the payoff matrix, is due to the similarity of the objective functions in the optimisations.

The similarity may be measured considering the significant correlation between the objective functions into consideration and/or due to the limited distance between the responses in the multiobjective space, always evaluated in pairs. If two objective functions present high correlation or 
small distance in the multi-objective space, it means that the trade-off between them is irrelevant and a multi-objective optimisation with this scenario may not achieve interest results, since the optimisation of one of these functions may reach near optimal results for the other one.

Consequently, in the present work, it is proposed a similarity analysis earlier to the multi-objective optimisation to deal with the imperfections of the objective functions. The objective is mainly related to the optimisation, and not to the modelling, since some functions measure different performance characteristics but with similar results in optimisation.

After achieving the Pareto frontier, it is sometimes required to rank the Pareto solutions through a mathematical criterion. The obtained Pareto solutions may serve to the experimenter as a set of possibilities to manage the process or product, by selecting the desired weight for each objective function in each specific situation of production scheduling. However, for the decision maker, a mathematical criterion may be useful to define the best compromise Pareto optimal solution. Considering the Euclidian distance $d_{j+}$ of each Pareto optimal solution $\overline{\mathbf{f}}_{j}^{*}$ to the utopia point $\overline{\mathbf{f}}^{\mathrm{U}}, j=1, \ldots, n_{\text {sub }}$, calculated according to the Equation 35 , the most compromise solution will be the one with lower $d_{j+}$, i.e., $\operatorname{Min}_{(j \in n s u b)}\left\{d_{j+}\right\}$ [63].

$$
d_{j+}=\sqrt{\sum_{i=1}^{m}\left(f_{i j}-f_{i}^{*}\right)^{2}}
$$

Next section presents the experimental procedure, describing equipment, material, tool, experimental design, method, and software.

\section{Experimental procedure}

Helical milling tests were carried out in workpieces of AISI H13 hardened steel using a CNC machining centre ROMI® Discovery 560 with numerical control Siemens ${ }^{\circledR}$ Sinumerik $810 \mathrm{D}$ in the machining laboratory of UFSJ. A hydraulic chuck BT-40 from DIN 1835-A/B standard, model 34.90.010 was used. The end mills were from Sandvik®, coromill plura code ISO/ANSI R215.H4 10050DAC03H 1610 with $D_{t}=10 \mathrm{~mm}$ diameter, $z=4$ and $a_{p(\text { máx })}=0.3 \mathrm{~mm}$, Sandvik grade GC 1610, ISO grade $\mathrm{H}$, with (Ti,Al)N 2 PVD coating. The experimental setup is detailed in Figure 7. 

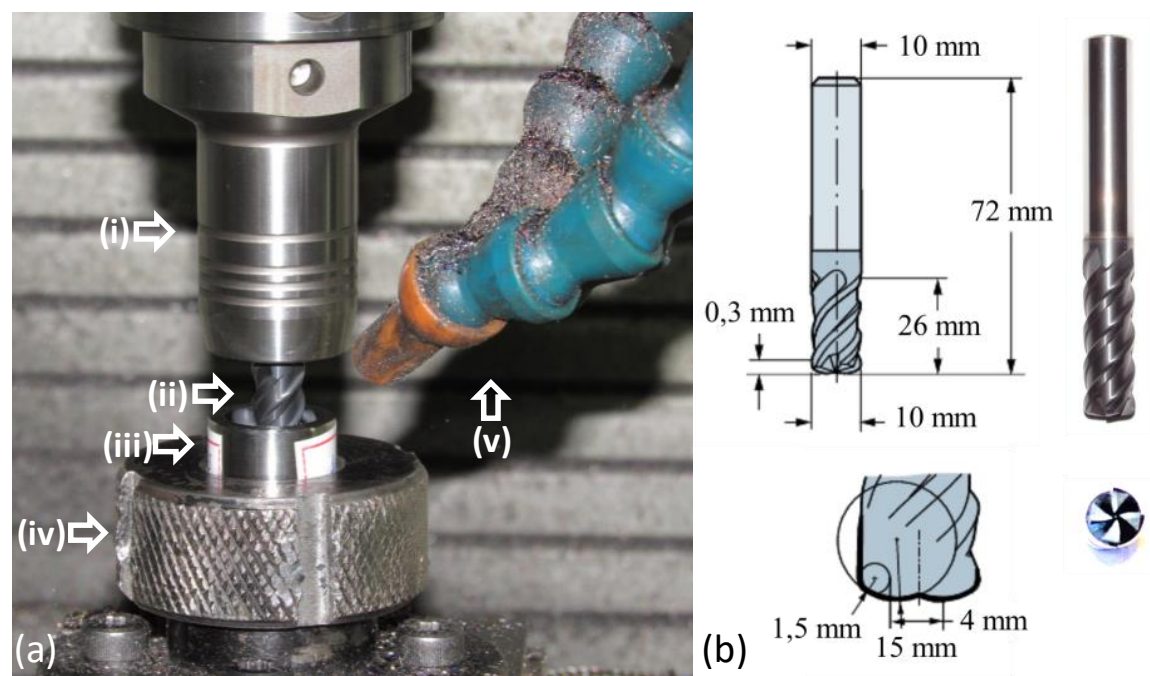

Figure 7. (a) Experimental setup: (i) hydraulic chuck, (ii) end mill, (iii) workpiece, (iv) fixture device and (v) air cooling system; (b) tool detail

As boreholes with diameter $D_{b}=18 \mathrm{~mm}$ were obtained, the helical diameter was $D_{h}=8 \mathrm{~mm}$ and the eccentricity of the tool centre point with regard to borehole centre point was $e=4 \mathrm{~mm}$. All boreholes were obtained in full, the radial cutting depth was constant, i.e., $a_{e}=\left(D_{b}{ }^{2} / 4 \times D_{h}\right)=18^{2} / 4 \times 8$ $=10.125 \mathrm{~mm}$. With $D_{b}=18 \mathrm{~mm}$ and $D_{t}=10 \mathrm{~mm}$ the ratio between peripheral and frontal cut was $\left(D_{b}^{2}-D_{t}^{2}\right) / D_{t}^{2}=\left(18^{2}-10^{2}\right) / 10^{2}=2.24$, supporting the peripheral cut, besides enabling good chip formation, chip evacuation and air cooling conditions.

To measure roughness of the borehole surfaces obtained by helical milling it was used a form talysurf profilometer from Taylor Hobson ${ }^{\circledR}$ with $50 \mathrm{~mm}$ transverse displacement, $\pm 0.5 \mathrm{~mm}$ measuring range, up to $10 \mathrm{~mm} / \mathrm{s}$ displacement velocity and up to $0.5 \mathrm{~mm} / \mathrm{s}$ measurement velocity. The profilometer is aided by computer and software ultra from Taylor Hobson®. The cut-off was $0.25 \mathrm{~mm}$. The measurements of roundness and cylindricity were carried out using a form talyround measurement system Talyround 131 from Taylor Hobson ${ }^{\circledR}$ with ruby probe, $2 \mathrm{~mm}$ high range, and high resolution of $6 \mathrm{~nm}$. The roundness measurement system is also aided by computer and software ultra from Taylor Hobson®.

Figure 8 illustrates the roughness and circularity measurement positions. For roughness, Figure 8(a) it was considered three radial positions angularly equidistant from $120^{\circ}$ and three positions (heights) in the axial directions, denoted beginning, middle and end. For roundness, it was considered 15 planes (heights) equidistant from $1.2 \mathrm{~mm}$. These positions were divided and denoted beginning, middle and end, as illustrated in Figure 8(b). For these two responses, these positions are related to the noise factor borehole surface height $\left(l_{b}\right)$. 

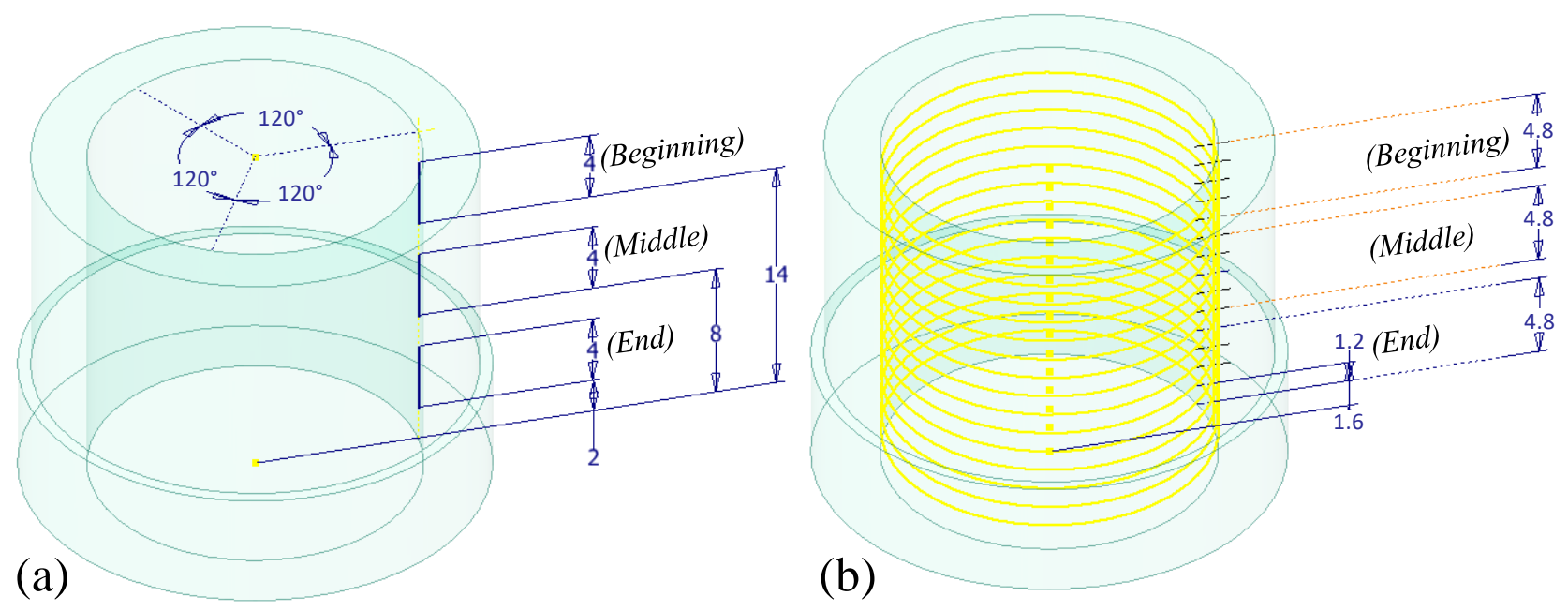

Figure 8. (a) Roughness; and (b) Roundness measurement planning

Control factors $(\mathbf{x})$ were considered according to helical milling kinematics as cutting velocity $\left(v_{c}\right)$, axial feed per tooth $\left(f_{z a}\right)$ and tangential feed per tooth $\left(f_{z t}\right)$. The control factors' levels of $f_{z a}$ and $f_{z t}$ were chosen through preliminary tests, respecting the tool constraint a $\mathrm{p}_{p(\max )}=0.3 \mathrm{~mm}$ while $v_{c}$ was chosen to regard tool manufacturer recommendations. Important noise factors were adopted to achieve robust levels of control factors with regard to noise factors influence in the outcomes. As argued in section 2, the noise factors $(\mathbf{z})$ were material hardness $\left(h_{d}\right)$, tool overhang length $\left(l_{t o}\right)$ and borehole height $\left(l_{b}\right)$. Control and noise factors' levels are in Table 1.

Table 1. Control and noise factors' levels

\begin{tabular}{ccccccc}
\hline \multirow{2}{*}{ Control factors } & \multicolumn{5}{c}{ Levels } & \multirow{2}{*}{ Units } \\
\cline { 2 - 6 } & -2.378 & -1 & 0 & 1 & 2.378 & \\
\hline$f_{z a}$ & 0.03 & 0.10 & 0.15 & 0.20 & 0.27 & $\mu \mathrm{m} / \mathrm{dente}$ \\
$f_{z t}$ & 0.03 & 0.10 & 0.15 & 0.20 & 0.27 & $\mathrm{~mm} / \mathrm{den}$ te \\
$v_{c}$ & 12.4 & 40.0 & 60.0 & 80.0 & 107.6 & $\mathrm{~m} / \mathrm{min}$ \\
\hline Control factors & & & & & & units \\
\hline$l_{t o}$ & - & 31 & 33 & 35 & - & $\mathrm{mm}$ \\
$h_{d}$ & - & 45 & 50 & 55 & - & HRC \\
$l_{b}$ & - & Beginning & Middle & End & - & - \\
\hline
\end{tabular}

The outcomes evaluated were the roughness parameters average surface roughness, $R_{a}$, maximum surface roughness, $R_{z}$, and the geometrical error parameters total roundness, $R_{0} n_{t}$, and total cylindricity, $C y l_{t}$. For all the statistical tests the significance level was $\alpha=0.05$. 
Analyses were carried out in Matlab®, Minitab® and spreadsheets software. The NNC routine was programmed in Matlab® considering $n_{s u b}=51$ solutions, therefore, with the increment $\delta_{r}$ $=0.02$. The algorithm sequential quadratic programming (SQP) was used with the number of maximum iterations equals to 1500 . The experimental constraint, $\mathbf{x} \in \Omega$ for the CCD design was according to the CCD design region, i.e., $f_{z a}{ }^{2}+f_{z t}^{2}+v_{c}{ }^{2} \leq \rho^{2}$, where $\rho$ is the radius of the spherical region, set to satisfy the design rotatability criteria. Considering three control factors, $\rho=\left(2^{k}\right)^{1 / 4}=$ $\left(2^{3}\right)^{1 / 4}=1.682$.

\section{Multi-objective robust optimization of borehole geometry and roughness of helical milling of AISI H13 hardened steel}

Figure 9 presents the flowchart for the multi-objective robust optimisation of helical milling process in AISI H13 hardened steel. The methodology may be applied in other manufacturing process optimisation. The flow chart of the method was divided into 6 steps denoted by the letters $\mathrm{A}$ to F, and each of these steps was subdivided into detailed substeps. The flowchart of the proposed method is elucidated as follows.

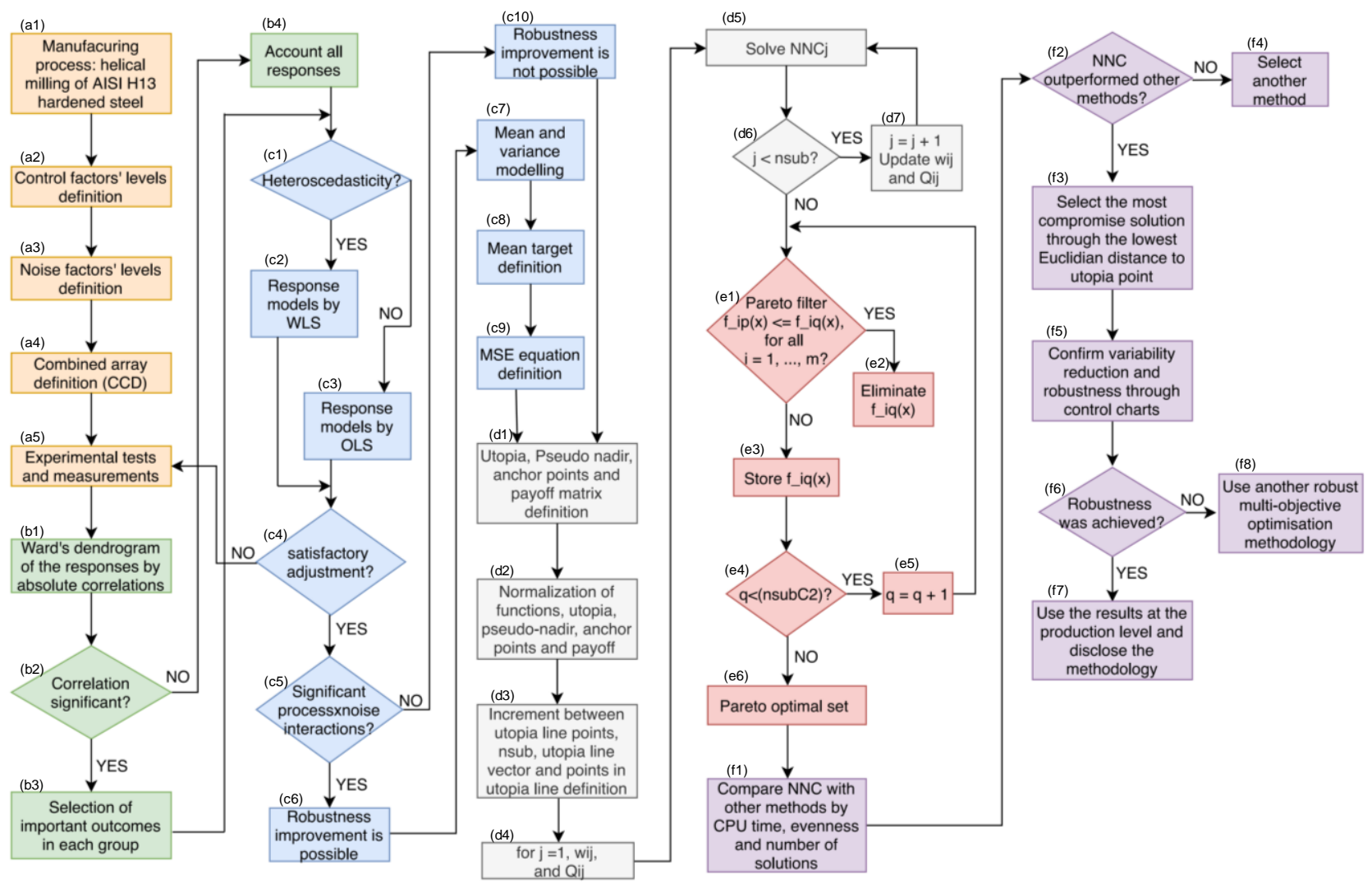


Figure 9. Flowchart for the multi-objective robust optimization of helical milling of AISI H13 hardened steel

\section{STEP A: EXPERIMENTAL TESTS.}

Substep a1: Manufacturing process selection. In the studied case it was the helical milling of AISI H13 hardened steel to evaluate borehole roughness and roundness.

Substep a2: Selection of control factors and its levels. In the present case, the control factors and its levels are depicted in Table 1.

Substep a3: Selection of noise factors and its levels. In the present case, the noise factors and its levels are also depicted in Table 1.

Substep a4: Combined array definition. The definition of the combined array may be provided considering a response surface design. In the present case it was used a CCD design with 32 tests considering a half fraction factorial with resolution $\mathrm{V}\left(n_{f}=2^{(k+r)-p}=2^{6-1}\right), 12$ tests in centre points $\left(n_{c}\right)$ and 6 axial points for control factors $\left(n_{a}=2 k=2 \times 3\right)$, with 50 tests in total. It is known that by adding more centre points the experimental error is better estimated. Considering the economy obtained by using the fractional design, the cost associated with 12 centre points, instead of 9 , as recommended, is negligible.

Substep a5: Experimental tests and measurements. In this work, helical milling cutting tests were performed and surface roughness and roundness measurements were performed.

\section{STEP B: SIMILARITY ANALYSIS.}

Substep b1: Dendrogram of Ward of the responses by absolute correlation. In the present case, it was considered different responses of microgeometrical error - roughness, and geometrical error roundness.

Substep b2: Correlation significant? The correlations between the responses separated in each group should be evaluated previously to the multi-objective optimisation.

Substep b3: Selection of important outcomes in each group. This substep is applied in the positive case of the substep b2, to avoid redundancy in multi-objective optimisation.

Substep b4: Account all responses. This step is aaplied in the negative case of this substep b2. In the absence of correlation, all the responses should be considered, since they present a substantial tradeoff.

STEP C: ROBUST MODELLING.

Substep c1: Heteroscedasticity? Homoscedasticity tests should be conducted to choose the better least squares approximations. 
Substep c2: Response models by WLS. The response models may be estimated by WLS in case of rejection of the null hypothesis of the homoscedasticity, i.e., in the positive case of substep c1.

Substep c3: Response models by OLS. In case of non-rejection of homoscedasticity, i.e., in the negative case of substep c1, OLS estimates may be used to estimate the response models.

Substep c4: Satisfactory adjustment? The goodness-of-fit measures should be evaluated. In the case of poor adjustment, return to substep a5.

Substep c5: Significant process $\times$ noise interactions? The significance of the process $\times$ noise interactions is important to enable the robust design.

Substep c6: Robustness improvement is possible. This assertion is done in the positive case of the substep c5.

Substep c7: Mean and variance modelling. Considering Equations 9 and 12, mean and variance models should be attained for each response model.

Substep c8: Mean target definition. For each response $T_{y i}=\underset{\mathbf{x} \in \Omega}{\operatorname{Min}}\left\{\underset{\mathbf{z}, \mathbf{z}}{E}\left[y_{i}(\mathbf{x}, \mathbf{z})\right]\right\}$.

Substep c9: $M S E$ equation definition. The $M S E$ equation is set up according to the Equation 15 considering mean, variance and target of mean obtained in substeps c7 and c8.

Substep c10: Robustness improvement is not possible. This assertion is done in the negative case of substep c5. Then, skip the substeps c6-c9, since it is not possible to transmit the variance with regard to noise factors to the control factors.

STEP D: MULTI-OBJECTIVE OPTIMISATION THROUGH NNC.

Substep d1: Utopia, Pseudo nadir, anchor points, and payoff matrix definition. These multi-objective definitions are important to start the optimisation. Define $\mathbf{f}^{\mathbf{U}}, \mathbf{f}^{\mathbf{P N}}, \mathbf{f}_{\mathrm{i}}{ }^{*}, i=1, \ldots, m$, and $\mathbf{\Phi}$.

Substep d2: Normalization of functions, utopia, pseudo-nadir, anchor points and payoff. The normalization, according to Equation 21, is important to avoid scale effects in optimisation.

Substep d3: Increment between utopia line points, utopia line vector and points in utopia line definition. Define $\delta_{r}, \eta_{r}, n_{s u b}, \overline{\mathbf{N}}_{\mathrm{r}}$ and $\overline{\mathbf{Q}}_{\mathrm{ij}}$.

Substep d4: For $j=1, w_{i j}$, and $\overline{\mathbf{Q}}_{\mathrm{ij}}$. For the first subproblem, $j=1$, define the weights, $w_{i j}, i=1, \ldots$, $m$ and the related point in the utopia line $\overline{\mathbf{Q}}_{\mathrm{ij}}$.

Substep d5: Solve $\mathrm{NNC}_{j}$. Through the formulation in Equations 24 and 25, or explicitly in Equations 33 and 34, solve $\mathrm{NNC}_{j}$ for $w_{i j}$, and $\overline{\mathbf{Q}}_{\mathrm{ij}}$.

Substep d6: $j<n_{s u b}$ ? While $j<n_{s u b}$, solve $\mathrm{NNC}_{j}$ by using the SQP algorithm.

Substep d7: $j=j+1$, update $w_{i j}$, and $\overline{\mathbf{Q}}_{\mathrm{ij}}$. In positive case of substep d6, actualize these metrics to perform the next optimisation through NNC. 
STEP E: PARETO FILTERING.

Substep e1: $\mathbf{f}_{i p} \leq \mathbf{f}_{i q}, i=1, \ldots, m$ ? In negative case of substep d6, i.e., after completing NNC optimisations, $j=1, \ldots, n_{\text {sub }}$, filtering the dominated solutions is necessary.

Substep e2: Eliminate $\mathbf{f}_{i q}$. In positive case of the substep e1, $\mathbf{f}_{i q}$ is dominated by $\mathbf{f}_{i p}$, since $\mathbf{f}_{i q}$ do not improve at least one objective function, $i=1, \ldots, m$.

Substep e3: Store $\mathbf{f}_{i q}$. In negative case of the substep e1, $\mathbf{f}_{i q}$ is a Pareto optimal solution and should be stored.

Substep e4: $q<\left(\begin{array}{c}n_{\text {sub }} \\ 2\end{array}\right)$ ? The number of comparisons performed in the Pareto filtering process is $\left(\begin{array}{c}n_{s u b} \\ 2\end{array}\right)$

Substep e5: $q=q+1$. In positive case of substep e4, actualize $q$ to continue the Pareto filtering.

Substep e6: Pareto optimal set. In the negative case of substep e4, stop filtering. The solutions stored in substep e3 constitute the Pareto set.

STEP F: CONFIRMATION OF THE OPTIMISATION

Substep f1: Compare NNC with other methods by CPU time, evenness and number of solutions.

Substep f2: NNC outperformed other methods? Answer considering the metrics proposed in substep f1.

Substep f3: Select the most compromise solution through the lowest Euclidian distance to the utopia point. In case positive of substep $\mathrm{f} 2$, calculate $d_{j+}$ in the normalized solution space and select the solution with minimum $d_{j+}$.

Substep f4: Select another method. In negative case of substep f2, select another multi-objective optimisation method.

Substep f5: Confirm variability reduction and robustness through control charts. I/MR control charts may be used to compare the experimental stage with the most compromise Pareto optimal solution simulated results.

Substep f6: Robustness was achieved? Evaluate the robustness achievement considering the reduction in the variability in the most compromise robust optimal solution.

Substep f7: Use the results at the production level and disclose the methodology. In the positive case of the substep f6, apply the results and methodology.

Substep f8: Use another multi-objective robust optimisation methodology. In negative case of the substep f6, select other methodology for multi-objective robust optimisation. 
It is important to discuss the computational complexity of the multi-objective optimisation method. Considering the steps D and E, which are related to NNC multi-objective optimisation and Pareto filtering respectively, there are important considerations with regard to computational cost. The computational complexity may be expressed using big-O notation in function of the number of subproblems $n_{\text {sub }}$.

For the initial definitions of the NNC method, steps d1-d4, the computational time is steady with regard to the $n_{s u b}$. Then the order of the initial definition is constant, i.e., $O(1)$. For the NNC loop, steps d5-d7, which is performed $n_{\text {sub }}$ times, the computational complexity is linear, i.e., of order $O\left(n_{\text {sub }}\right)$. In each step of the NNC loop the SQP algorithm is performed. For the Pareto filtering process, steps e1-e6, it is carried out $\left(\begin{array}{c}n_{\text {sub }} \\ 2\end{array}\right)=\left(n_{\text {sub }}^{2}-n_{\text {sub }}\right) / 2$ comparisons among the solutions. Consequently, the computational complexity is quadratic, $O\left(n_{\text {sub }}^{2}\right)$. However, since this procedure is a simple comparison, for a feasible desired number of Pareto solutions, $n_{s u b}$, the filtering process may present lower time than the NNC loop. This will be discussed considering the present application in section 9.4 .

\section{Results and discussion}

Table 2 presents the experimental results for the 50 tests of helical milling of AISI H13 hardened steel considering a CCD design. The roundness, $R_{o n}$, results were from 5.28 to $16.86 \mu \mathrm{m}$. The cylindricity, $C y l_{t}$, which accounts not only the roundness deviation but also the eccentricity, considering the measurement method of the form measurement system used, was from 6.92 to 21.01 $\mu \mathrm{m}$. Iyer et al. [9] achieved Ron $_{t}=10 \mu \mathrm{m}$ in the helical milling of AISI D2 hardened steel to achieve boreholes of $16 \mathrm{~mm}$ with a solid carbide end mill. About roughness it was obtained from 0.19 to 0.44 $\mu \mathrm{m}$ for $R_{a}$ and 1.27 to $2.45 \mu \mathrm{m}$ for $R_{z}$, attesting the excellent roughness results obtained. In the present work, the obtained roughness levels may be compared with the grinding process.

Table 2. Experimental results

\begin{tabular}{|c|c|c|c|c|c|c|c|c|c|c|}
\hline Std & $f_{z a}$ & $f_{z t}$ & $v_{c}$ & $l_{\text {to }}$ & $h_{d}$ & $l_{b}$ & Ron $_{t}$ & $C y l_{t}$ & $R_{a}$ & $R_{z}$ \\
\hline Ord & {$[\mu \mathrm{m} /$ dente $]$} & [mm/dente] & {$[\mathrm{m} / \mathrm{min}]$} & [mm] & HRc & - & {$[\mu \mathrm{m}]$} & {$[\mu \mathrm{m}]$} & {$[\mu \mathrm{m}]$} & {$[\mu \mathrm{m}]$} \\
\hline 1 & 0.1 & 0.1 & 40 & 31 & 45 & -1 & 7.37 & 10.76 & 0.22 & 1.38 \\
\hline 2 & 0.2 & 0.1 & 40 & 31 & 45 & 1 & 7.53 & 9.46 & 0.27 & 1.66 \\
\hline 3 & 0.1 & 0.2 & 40 & 31 & 45 & 1 & 8.05 & 11.87 & 0.23 & 1.41 \\
\hline
\end{tabular}




\begin{tabular}{|c|c|c|c|c|c|c|c|c|c|c|}
\hline 4 & 0.2 & 0.2 & 40 & 31 & 45 & -1 & 7.70 & 10.50 & 0.26 & 1.65 \\
\hline 5 & 0.1 & 0.1 & 80 & 31 & 45 & 1 & 9.04 & 12.82 & 0.29 & 1.68 \\
\hline 6 & 0.2 & 0.1 & 80 & 31 & 45 & -1 & 10.02 & 11.77 & 0.23 & 1.40 \\
\hline 7 & 0.1 & 0.2 & 80 & 31 & 45 & -1 & 8.87 & 10.44 & 0.23 & 1.44 \\
\hline 8 & 0.2 & 0.2 & 80 & 31 & 45 & 1 & 7.86 & 9.74 & 0.26 & 1.45 \\
\hline 9 & 0.1 & 0.1 & 40 & 35 & 45 & 1 & 11.04 & 12.62 & 0.27 & 1.62 \\
\hline 10 & 0.2 & 0.1 & 40 & 35 & 45 & -1 & 12.94 & 15.53 & 0.27 & 1.70 \\
\hline 11 & 0.1 & 0.2 & 40 & 35 & 45 & -1 & 9.86 & 12.22 & 0.31 & 1.88 \\
\hline 12 & 0.2 & 0.2 & 40 & 35 & 45 & 1 & 9.04 & 11.89 & 0.30 & 1.83 \\
\hline 13 & 0.1 & 0.1 & 80 & 35 & 45 & -1 & 10.76 & 12.46 & 0.31 & 1.74 \\
\hline 14 & 0.2 & 0.1 & 80 & 35 & 45 & 1 & 12.48 & 14.86 & 0.35 & 1.96 \\
\hline 15 & 0.1 & 0.2 & 80 & 35 & 45 & 1 & 10.18 & 12.82 & 0.36 & 1.95 \\
\hline 16 & 0.2 & 0.2 & 80 & 35 & 45 & -1 & 6.95 & 8.90 & 0.25 & 1.53 \\
\hline 17 & 0.1 & 0.1 & 40 & 31 & 55 & 1 & 8.50 & 13.65 & 0.22 & 1.29 \\
\hline 18 & 0.2 & 0.1 & 40 & 31 & 55 & -1 & 7.65 & 11.40 & 0.25 & 1.55 \\
\hline 19 & 0.1 & 0.2 & 40 & 31 & 55 & -1 & 8.37 & 11.19 & 0.21 & 1.32 \\
\hline 20 & 0.2 & 0.2 & 40 & 31 & 55 & 1 & 15.12 & 21.01 & 0.24 & 1.44 \\
\hline 21 & 0.1 & 0.1 & 80 & 31 & 55 & -1 & 6.90 & 8.38 & 0.22 & 1.35 \\
\hline 22 & 0.2 & 0.1 & 80 & 31 & 55 & 1 & 13.74 & 18.35 & 0.26 & 1.52 \\
\hline 23 & 0.1 & 0.2 & 80 & 31 & 55 & 1 & 8.06 & 11.74 & 0.25 & 1.49 \\
\hline 24 & 0.2 & 0.2 & 80 & 31 & 55 & -1 & 8.04 & 11.72 & 0.23 & 1.42 \\
\hline 25 & 0.1 & 0.1 & 40 & 35 & 55 & -1 & 12.26 & 14.41 & 0.21 & 1.34 \\
\hline 26 & 0.2 & 0.1 & 40 & 35 & 55 & 1 & 7.98 & 10.90 & 0.22 & 1.34 \\
\hline 27 & 0.1 & 0.2 & 40 & 35 & 55 & 1 & 7.39 & 11.89 & 0.27 & 1.56 \\
\hline 28 & 0.2 & 0.2 & 40 & 35 & 55 & -1 & 5.55 & 12.06 & 0.28 & 1.64 \\
\hline 29 & 0.1 & 0.1 & 80 & 35 & 55 & 1 & 16.86 & 20.68 & 0.36 & 1.95 \\
\hline 30 & 0.2 & 0.1 & 80 & 35 & 55 & -1 & 9.83 & 14.05 & 0.23 & 1.37 \\
\hline 31 & 0.1 & 0.2 & 80 & 35 & 55 & -1 & 10.60 & 13.41 & 0.26 & 1.58 \\
\hline 32 & 0.2 & 0.2 & 80 & 35 & 55 & 1 & 7.59 & 19.29 & 0.26 & 1.59 \\
\hline 33 & 0.031 & 0.15 & 60 & 33 & 50 & 0 & 9.22 & 10.30 & 0.19 & 1.28 \\
\hline 34 & 0.269 & 0.15 & 60 & 33 & 50 & 0 & 11.99 & 13.10 & 0.27 & 1.62 \\
\hline 35 & 0.15 & 0.031 & 60 & 33 & 50 & 0 & 8.78 & 10.24 & 0.44 & 2.45 \\
\hline 36 & 0.15 & 0.269 & 60 & 33 & 50 & 0 & 6.06 & 6.92 & 0.22 & 1.32 \\
\hline
\end{tabular}




\begin{tabular}{lllllllllll}
37 & 0.15 & 0.15 & 12.43 & 33 & 50 & 0 & 11.42 & 12.80 & 0.22 & 1.46 \\
38 & 0.15 & 0.15 & 107.57 & 33 & 50 & 0 & 9.27 & 10.87 & 0.34 & 1.77 \\
39 & 0.15 & 0.15 & 60 & 33 & 50 & 0 & 6.57 & 8.49 & 0.30 & 1.72 \\
40 & 0.15 & 0.15 & 60 & 33 & 50 & 0 & 13.40 & 14.23 & 0.24 & 1.47 \\
41 & 0.15 & 0.15 & 60 & 33 & 50 & 0 & 12.57 & 14.19 & 0.28 & 1.64 \\
42 & 0.15 & 0.15 & 60 & 33 & 50 & 0 & 6.60 & 7.41 & 0.23 & 1.34 \\
43 & 0.15 & 0.15 & 60 & 33 & 50 & 0 & 8.33 & 10.10 & 0.22 & 1.29 \\
44 & 0.15 & 0.15 & 60 & 33 & 50 & 0 & 8.94 & 10.64 & 0.22 & 1.27 \\
45 & 0.15 & 0.15 & 60 & 33 & 50 & 0 & 5.28 & 7.03 & 0.28 & 1.70 \\
46 & 0.15 & 0.15 & 60 & 33 & 50 & 0 & 8.07 & 9.04 & 0.28 & 1.69 \\
47 & 0.15 & 0.15 & 60 & 33 & 50 & 0 & 6.34 & 7.44 & 0.26 & 1.57 \\
48 & 0.15 & 0.15 & 60 & 33 & 50 & 0 & 15.09 & 16.41 & 0.29 & 1.69 \\
49 & 0.15 & 0.15 & 60 & 33 & 50 & 0 & 5.88 & 7.28 & 0.28 & 1.59 \\
50 & 0.15 & 0.15 & 60 & 33 & 50 & 0 & 6.66 & 7.89 & 0.31 & 1.78 \\
\hline
\end{tabular}

\subsection{Similarity analysis}

Figure 10 shows de Ward's dendogram with absolute correlation as similarity measure. It can be observed that one group is formed for the roughness responses with similarity equals to $97.12 \%$, while the other group is composed for the geometrical error outcomes with similarity equals to $82.04 \%$. For the two groups, the correlation was positive. Considering the high similarity level, it is impractical to consider all these responses in a multi-objective optimization scenario, since high correlated outcomes should present similar results in the optimization. Therefore, in each group one response was selected considering its importance and the goodness-of-fit measures, as presented in the next sections, to assure good optimization results and reproducibility.

In the group of roughness $R_{a}$ was selected, since it is the outcome defined by standards to determine the state of surfaces in technical drawings being the most important parameter in investigations of finishing manufacturing process. Also, as will be presented in section 7.3, $R_{a}$ presented better results than $R_{z}$ with regard to goodness-of-fit measures. With regard to the group of geometrical error outcomes, Ron $_{t}$ was selected since it is frequently addressed as an index of geometrical error in machined boreholes. In addition, Ron $_{t}$ presented better results of goodness-of-fit statistics when compared to $C y l_{t}$, as shown in section 7.2. 


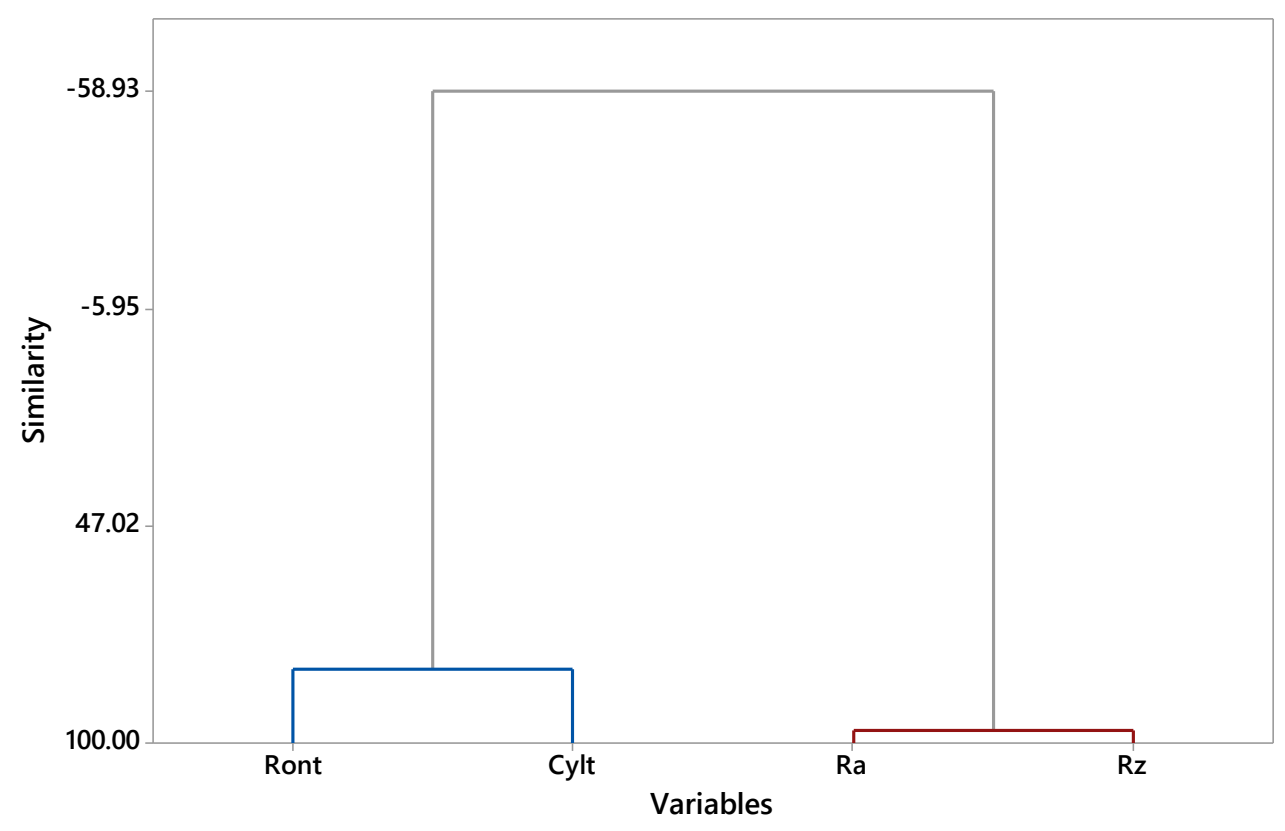

Figure 10. Ward's dendogram for $\operatorname{Ron}_{t}, C y l_{t}, R_{a}$ and $R_{z}$

\subsection{Geometrical error on helical milling of AISI H13 hardened steel}

Figure 11 presents one of the roundness measurements results in the workpiece of test 36. Total roundness is an important index of geometrical error of boreholes. More details about measurement and calculation can be found in SUN [64]. In this helical milling test, the following levels of process parameters were applied $\mathbf{x}_{36}=[0.15 \mu \mathrm{m} /$ tooth; $0.269 \mathrm{~mm} /$ tooth; $60 \mathrm{~m} / \mathrm{min}]$, with noise factors levels fixed in $\mathbf{z}_{36}=\left[33 \mathrm{~mm} ; 50 \mathrm{HRC}\right.$; 0]. The height $l_{b}=0$ is in the middle of the borehole as illustrated in Figure 8. 


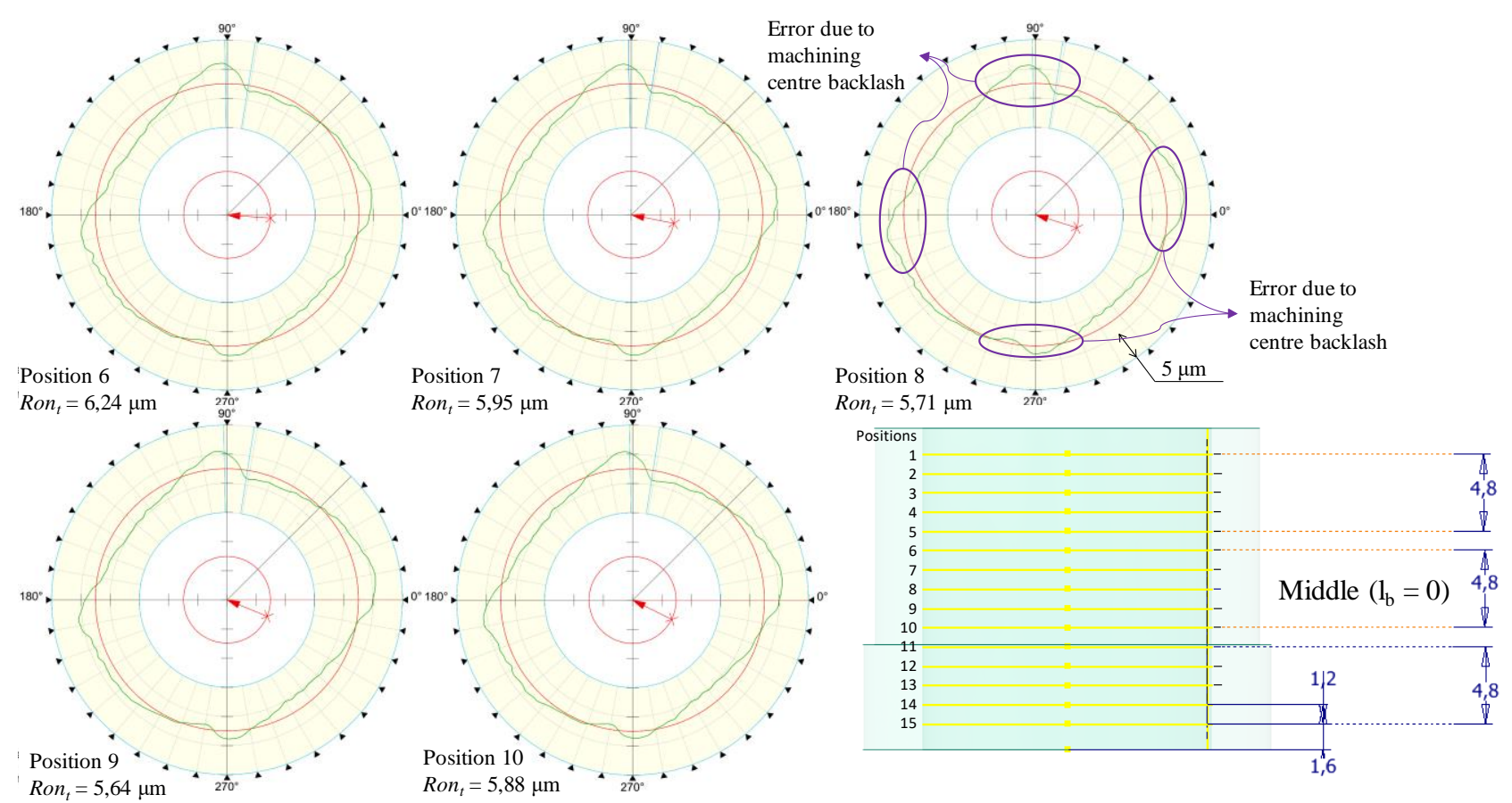

Figure 11. One of the roundness measurements for test 36

Taking arbitrarily the measurement in position 8, in Figure 11, it can be observed the presence of protuberances in the quadrants of the roundness profile. As observed by Costa et al. [49], these geometrical errors are due to the machining centre axes' backlashes. The geometrical error in circular trajectories appears during the change of the movement direction of the machine table during the interpolation of the axes $x$ and $y$ [66]. The backlashes were identified in the tests which presented Ron $_{t}$ smaller than $11 \mu \mathrm{m}$. This reflects the good geometrical quality of the obtained boreholes, and the machining centre limit in achieving better results, since with the absence of backlash improved results would be achieved.

Besides the total roundness, the total cylindricity was also evaluated. The cylindricity measurement method used, inherent to the measurement system, takes into account not only the cylindrical form error but also the eccentricity error, by calculating a least squares centre line considering the centre points of the roundness measurements planes considering the measurement planes. Then, to minimize cylindricity implies minimizing the cylindrical form error, besides, the eccentricity among the measured planes of the borehole.

Figure 12 illustrates the graphical result of one of the cylindricity measurements for the test number 36, with regard to roundness measurements plotted in Figure 11. It can be observed in a 3D view the protuberances angularly equidistant from $90^{\circ}$ due to the backlashes of the machining centre axes. 


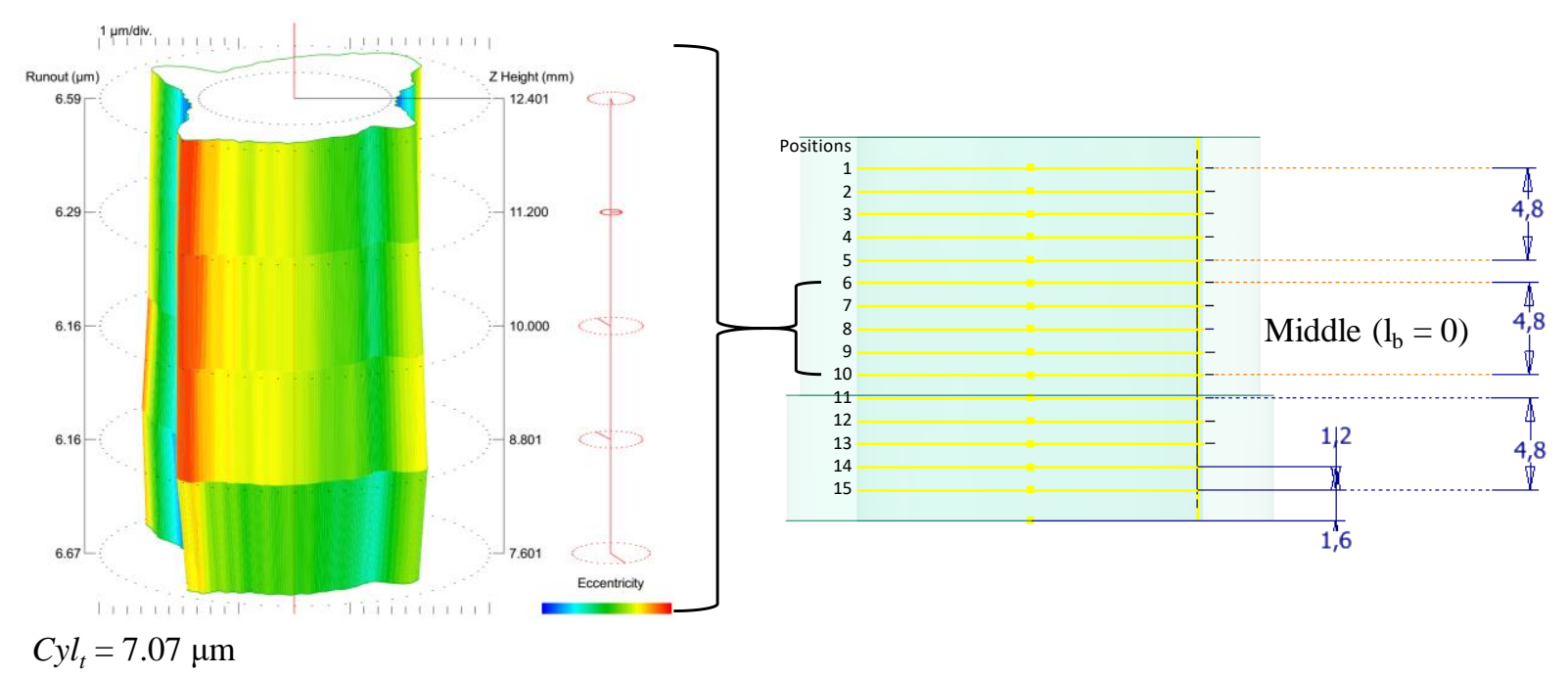

Figure 12. Cylindricity measurement result for test 36

Table 3 presents the ANOVA results for Ron $_{t}$ and $C y l_{t}$ which may be analysed together with the main and quadratic effects and interaction effects plotted in Figures 13 and 14. For Ron , with regard to linear process effects, $f_{z t}$ was the unique control factor with significance, with a negative effect, as observed graphically in Figure 13. Observing graphically this effect mixed with the quadratic effect, also statistically significant, when increasing $f_{z t}$ until the level $0.11 \mu \mathrm{m} /$ tooth, $_{\text {Ron }}$ slightly increases, while above this value, the increase of $f_{z t}$ resulted in the decrease of Ron $_{t}$. The factors $f_{z a}$ and $v_{c}$ also presented quadratic effects with convexity upward. With regard to noise factors, only $l_{t o}$ presented significance in the linear effect so that the increase in $l_{t o}$ caused the increase in $\operatorname{Ron}_{t}$. These effects can also be confirmed through the signal of the regression coefficients presented hereafter.

For $C y l_{t}$, with regard to the linear process effects, only $v_{c}$ was significant. The quadratic effect for this factor also was the only one significant with convexity downward, with minimum cylindricity achieved nearby the centre point level $v_{c}=60 \mathrm{~m} / \mathrm{min}$. The factors $f_{z a}$ and $f_{z t}$ were not significant individually. However, graphically may be evaluated the effect of these parameters. The significance is related to the experimental error and Box and Drapper [20] claim that process knowledge and graphical analysis may be important to understand the results.

With regard to noise factors effects, $l_{t o}$ was the only one significant in $\operatorname{Ron}_{t}$, while for $C y l_{t}$ all noise factors were statistically significant. The increase of tool overhang length, $l_{t o}$, resulted in the increase of Ront and Cylt, which may be related to the tool deflection increase, which may result in vibrations. The increase of material hardness, $h_{d}$, entails the increase of $C y l_{t}$, confirming the difficulty in obtaining tight form tolerances in hard machining. Finally, by moving to the end of the borehole, considering the noise factor $l_{b}$, resulted in higher levels of $C y l_{t}$. The noise factors effects 
are important to enable the RPD. However, the process $\times$ noise interactions are fundamental to achieve robustness.

Table 3. ANOVA for $\operatorname{Ron}_{t}$ and $C y l_{t}$

\begin{tabular}{|c|c|c|c|c|c|}
\hline \multirow{2}{*}{ Source } & Ron $_{t}$ & $C y l_{t}$ & \multirow{2}{*}{ Source } & Ron $_{t}$ & $C y l_{t}$ \\
\hline & P-value & P-value & & P-value & P-value \\
\hline Model & $* 0.000$ & 0.000 & Interaction & 0.000 & 0.004 \\
\hline Linear & 0.000 & 0.000 & $f_{z a} \times f_{z t}$ & 0.942 & 0.274 \\
\hline$f_{z a}$ & 0.510 & 0.253 & $f_{z a} \times v_{c}$ & 0.697 & 0.109 \\
\hline$f_{z t}$ & 0.000 & 0.317 & $f_{z t} \times v_{c}$ & 0.168 & 0.008 \\
\hline$v_{c}$ & 0.359 & 0.015 & $f_{z a} \times l_{t o}$ & 0.004 & 0.070 \\
\hline$l_{t o}$ & 0.011 & 0.002 & $f_{z a} \times h_{d}$ & 0.250 & 0.009 \\
\hline$h_{d}$ & 0.351 & 0.005 & $f_{z a} \times l_{b}$ & 0.484 & 0.410 \\
\hline$l_{b}$ & 0.073 & 0.001 & $f_{z t} \times l_{t o}$ & 0.007 & 0.419 \\
\hline Quadratic & 0.000 & 0.006 & $f_{z t} \times h_{d}$ & 0.383 & 0.468 \\
\hline$f_{z a} \times f_{z a}$ & 0.006 & 0.051 & $f_{z t} \times l_{b}$ & 0.351 & 0.738 \\
\hline$f_{z t} \times f_{z t}$ & 0.001 & 0.983 & $v_{c} \times l_{t o}$ & 0.518 & 0.045 \\
\hline$v_{c} \times v_{c}$ & 0.019 & 0.004 & $v_{c} \times h_{d}$ & 0.630 & 0.046 \\
\hline$R^{2}$ & $99.98 \%$ & $99.15 \%$ & $v_{c} \times l_{b}$ & 0.116 & 0.002 \\
\hline$R_{a d j}^{2}$ & $99.97 \%$ & $98.51 \%$ & LOF & 0.362 & 0.202 \\
\hline$R_{\text {prev }}^{2}$ & $98.78 \%$ & $58.29 \%$ & $S$ & 1.114 & 1.068 \\
\hline
\end{tabular}

Concerning the interaction effects for Ron $_{t}$, there was no significant interaction between process factors, while for the process $\times$ noise interactions $f_{z a} \times l_{t o}$ and $f_{z t} \times l_{t o}$ were statistically significant. Tool overhang length variation may cause tool deflection and vibrations with resulting geometrical error. Then, it may be achieved levels of $f_{z a}$ and $f_{z t}$ which are robust to $l_{t o}$ variation. These levels will be achieved with robust multi-objective optimization.

About the interaction effects for $C y l_{t}$, the interaction between process factors $f_{z t} \times v_{c}$ was statistically significant. The effect of $f_{z t}$ is positive when $v_{c}$ is low. However, when $v_{c}$ is high, the $f_{z t}$ effect is negative on $C y l_{t}$. With regard to process $\times$ noise interactions, $f_{z a} \times l_{t o}, f_{z a} \times h_{d}, v_{c} \times l_{t o}, v_{c} \times h_{d}$ and $v_{c} \times l_{b}$ were statistically significant. These interactions may be graphically analysed to view levels of process factors which makes the form error responses insensitive to noise variation. With regard to the first significant process $\times$ noise interaction, when increasing $f_{z a}$, the response $C y l_{t}$ becomes robust 
with regard to noise factor $l_{t o}$ variation, as can be confirmed graphically in Figure 14. But, taking with consideration the interaction $f_{z a} \times h_{d}$, low levels of $f_{z a}$ makes the outcome $C y l_{t}$ insensitive to noise factor $h_{d}$. This trade-off between different process $\times$ noise interaction involving a fixed process factor is complex to manage but can be solved through optimization. The process $\times$ noise interactions involving the factor $v_{c}$ indicates lower levels of $v_{c}$ to make the outcome $C y l_{t}$ insensitive with regard to $l_{t o}, h_{d}$ and $l_{b}$, as observed graphically in Figure 14.

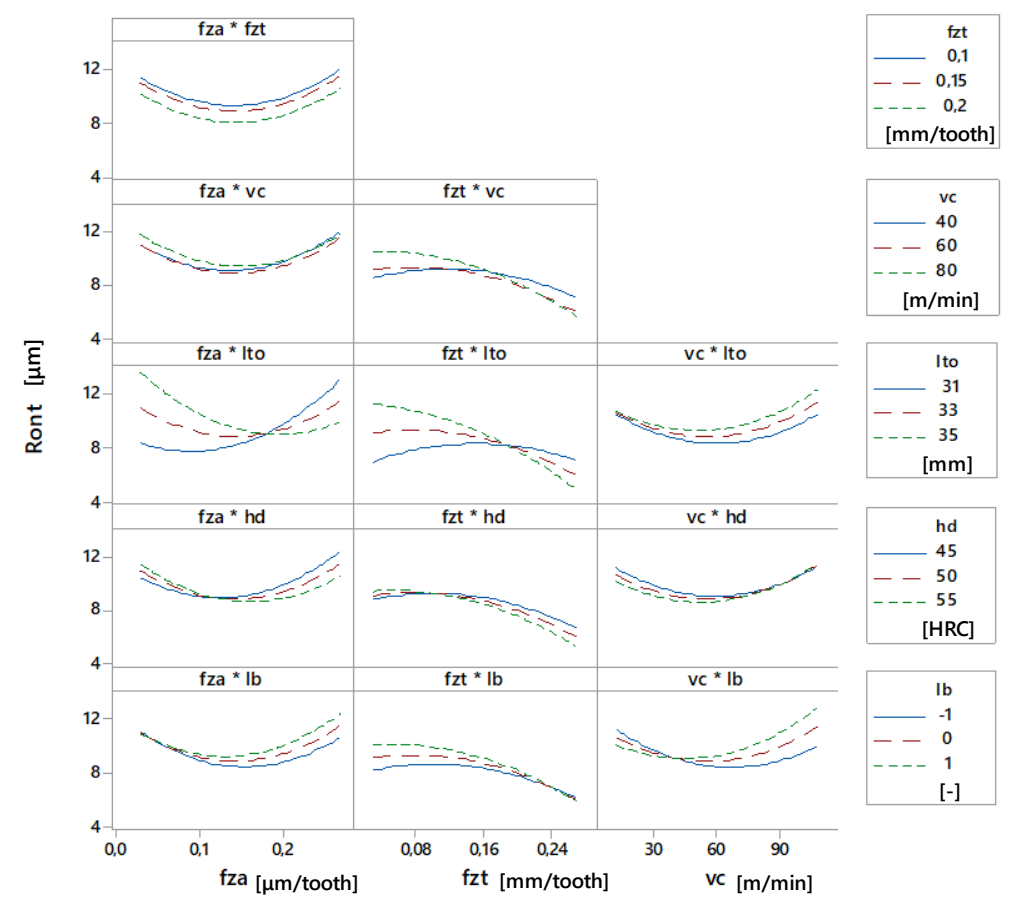

Figure 13. Interaction effects plots for $\operatorname{Ron}_{t}$ 


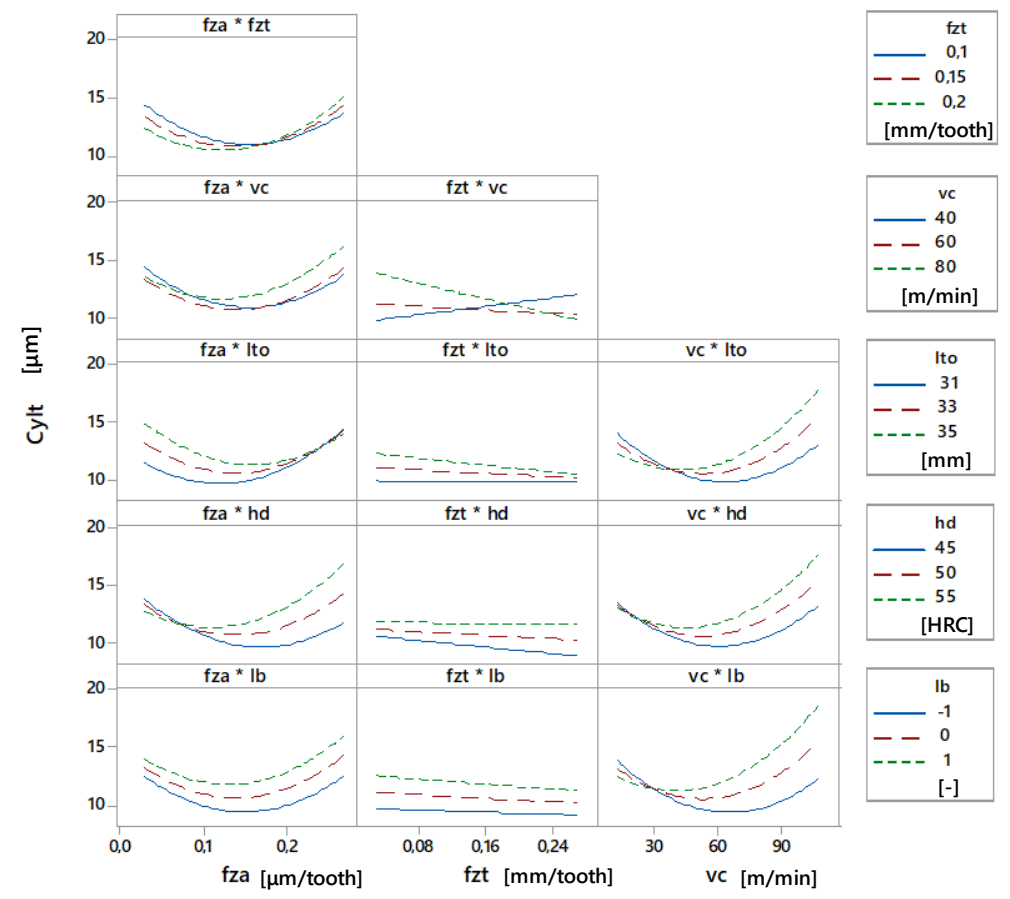

Figure 14. Interaction effects plots for $C y l_{t}$

Equation 36 presents the response model for Ron $_{t}$ obtained through WLS. The homoscedasticity test results are in Appendix B. The significance of the coefficients is summarized in Table 3 and the signal of the effects can be confirmed graphically in Figure 13. The model error estimated by ANOVA was $\hat{\sigma}_{R o n_{t}}=1.095$, with $R_{a j}{ }^{2}=99.97 \%$ and $R_{p r e v}{ }^{2}=98.78 \%$, assuring the good variability explanation and prediction capability. The lack-of-fit (LOF) reported p-value $=0.362$, guaranteeing no lack-of-fit for the response model for Ron $_{t}$.

$$
\begin{aligned}
\operatorname{Ron}_{t}(\mathbf{x}, \mathbf{z}) & =8,8570+0.1090 \times f_{z a}-0,6456 \times f_{z t}+0,1534 \times v_{c}+0,4097 \times f_{z a}{ }^{2}-0,2232 \times f_{z t}{ }^{2} \\
& +0,3756 \times v_{c}{ }^{2}-0,0183 \times f_{z a} \times f_{z t}-0,0971 \times f_{z a} \times v_{c}-0,3514 \times f_{z t} \times v_{c}+0,5178 \times l_{t o} \\
& -0,1989 \times h_{d}+0,4146 \times l_{b}-0,8666 \times f_{z a} \times l_{t o}-0,3000 \times f_{z a} \times h_{d}+0,1948 \times f_{z a} \times l_{b} \\
& -0,6649 \times f_{z t} \times l_{t o}-0,2065 \times f_{z t} \times h_{d}-0,2138 \times f_{z t} \times l_{b}+0,1623 \times v_{c} \times l_{t o} \\
& +0,1209 \times v_{c} \times h_{d}+0,4097 \times v_{c} \times l_{b}
\end{aligned}
$$

Equation 37 presents the response model for $C y l_{t}$ in function of process and noise factors obtained through WLS. The homoscedasticity test results are in Appendix B. The intensity and signal of the effects, observed graphically in Figure 14 may be confirmed by analysing the coefficients of this model. The experimental error of the response model of $C y l_{t}$ estimated through ANOVA was $\hat{\sigma}_{C y l_{t}}=$ 1.068 , with $R_{a j}{ }^{2}=98.51 \%$ and $R_{p r e v}{ }^{2}=58.29 \%$ assuring good variability explanation capability, 
however, with modest prediction capability. The LOF test resulted in the p-value $=0.202$, guaranteeing no lack-of-fit of the model, considering the significance level adopted, $\alpha=0.05$.

$$
\begin{aligned}
C y l_{t}(\mathbf{x}, \mathbf{z}) & =10,6612+0.2169 \times f_{z a}-0,1925 \times f_{z t}+0,4568 \times v_{c}+0,5373 \times f_{z a}{ }^{2}+0,0054 \times f_{z t}{ }^{2} \\
& +0,6384 \times v_{c}{ }^{2}+0,3543 \times f_{z a} \times f_{z t}+0,3460 \times f_{z a} \times v_{c}-0,6489 \times f_{z t} \times v_{c}+0,7425 \times l_{t o} \\
& +1,0039 \times h_{d}+1,2281 \times l_{b}-0,4071 \times f_{z a} \times l_{t o}+0,6378 \times f_{z a} \times h_{d}+0,2079 \times f_{z a} \times l_{b} \\
& -0,1797 \times f_{z t} \times l_{t o}-0,1707 \times f_{z t} \times h_{d}-0,0753 \times f_{z t} \times l_{b}+0,6587 \times v_{c} \times l_{t o} \\
& +0,5090 \times v_{c} \times h_{d}+0,8056 \times v_{c} \times l_{b}
\end{aligned}
$$

To achieve robust models with regard to noise factors the RPD was performed. It was considered $E\left[z_{j}\right]=0$ to attain a mean model in function of process factors only. By performing the error propagation with regard to noise factors, a variance model was achieved also in function of process factors. The models for mean and variance of $\operatorname{Ron}_{t}, E\left[\operatorname{Ron}_{t}\right]$ and $\operatorname{Var}\left[\operatorname{Ron}_{t}\right]$, in function of control factors, are depicted in Equations 38 and 39 to enable achieving robust levels of control factors concerning noise factors variation in the total roundness of the boreholes. The response surfaces for $E\left[\operatorname{Ron}_{t}\right]$ and $\operatorname{Var}\left[\operatorname{Ron}_{t}\right]$ are plotted in Figure 15 under different perspectives, with the variable in labels at centre point level.

$$
\begin{gathered}
E\left[\operatorname{Ron}_{t}(\mathbf{x}, \mathbf{z})\right]=8,8570+0.1090 \times f_{z a}-0,6456 \times f_{z t}+0,1534 \times v_{c}+0,4097 \times f_{z a}{ }^{2} \\
\quad-0,2232 \times f_{z t}{ }^{2}+0,3756 \times v_{c}{ }^{2}-0,0183 \times f_{z a} \times f_{z t}-0,0971 \times f_{z a} \times v_{c}-0,3514 \times f_{z t} \times v_{c} \\
\operatorname{Var}\left[\operatorname{Ron}_{t}(\mathbf{x}, \mathbf{z})\right]=1,6796-0,6166 \times f_{z a}-7838 \times f_{z t}+04597 \times v_{c}+0,8790 \times f_{z a}{ }^{2}+0,5305 \times f_{z t}{ }^{2} \\
+0,2088 \times v_{c}{ }^{2}+1,1931 \times f_{z a} \times f_{z t}-0,1943 \times f_{z a} \times v_{c}-0,4410 \times f_{z t} \times v_{c}
\end{gathered}
$$

Equations 40 and 41 present the models of mean and variance for $C y l_{t}$. The response surface plots related to these models are presented in Figure 16 under different perspectives, with the variable in labels at centre point level.

$$
\begin{gathered}
E\left[C y l_{t}(\mathbf{x}, \mathbf{z})\right]=10,6612+0.2169 \times f_{z a}-0,1925 \times f_{z t}+0,4568 \times v_{c}+0,5373 \times f_{z a}{ }^{2}+0,0054 \times f_{z t}{ }^{2} \\
\quad+0,6384 \times v_{c}{ }^{2}+0,3543 \times f_{z a} \times f_{z t}+0,3460 \times f_{z a} \times v_{c}-0,6489 \times f_{z t} \times v_{c} \\
\operatorname{Var}\left[\operatorname{Cyl} l_{t}(\mathbf{x}, \mathbf{z})\right]=4,2073+1,1865 \times f_{z a}-0,1090 \times f_{z t}+3,9786 \times v_{c}+0,6157 \times f_{z a}{ }^{2}+0,0671 \times f_{z t}{ }^{2} \\
+1,3418 \times v_{c}{ }^{2}+0,3328 \times f_{z a} \times f_{z t}+0,4478 \times f_{z a} \times v_{c}-0,1843 \times f_{z t} \times v_{c}
\end{gathered}
$$



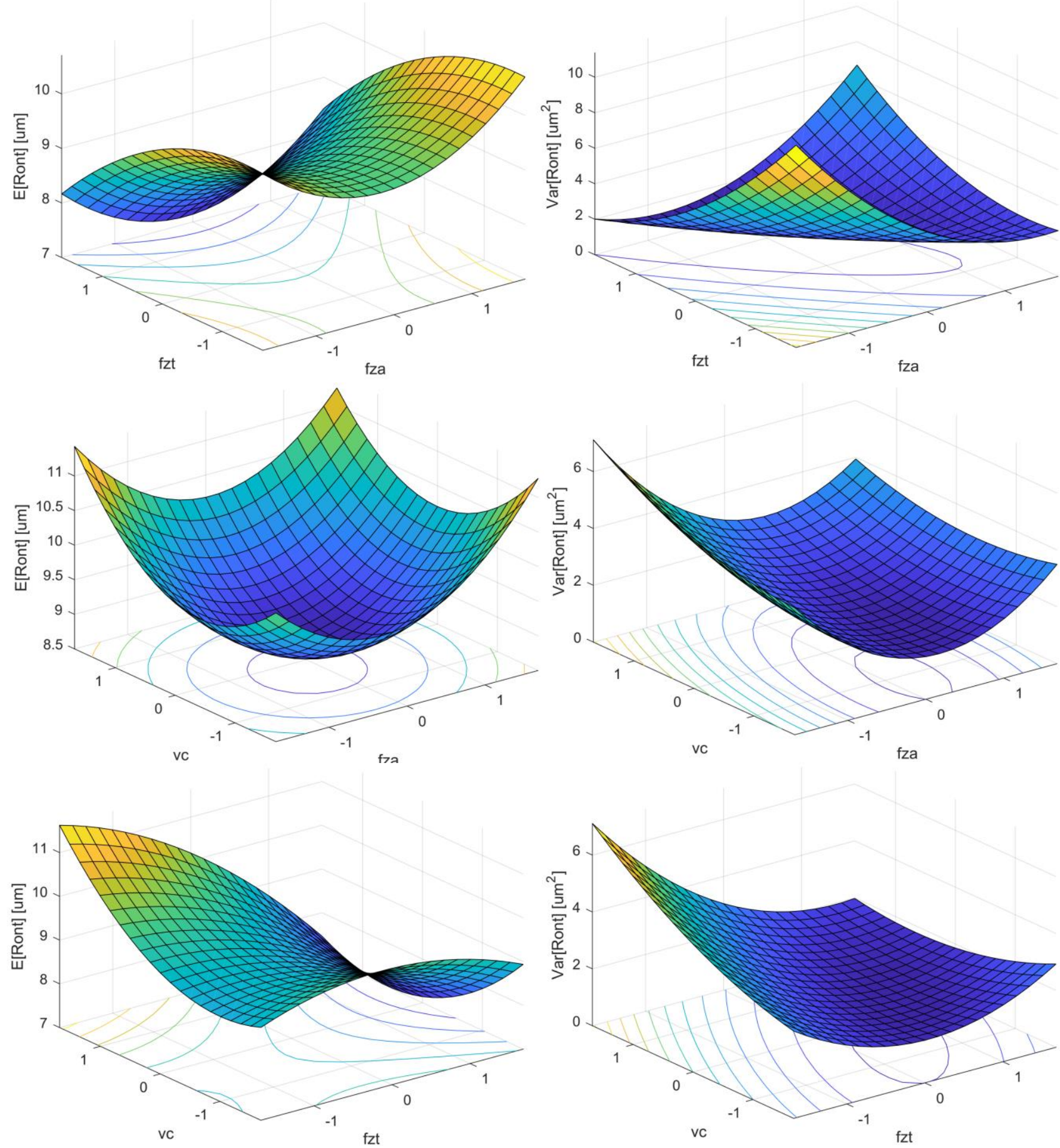

Figure 15. Response surfaces for $E\left[\operatorname{Ron}_{t}\right]$ and $\operatorname{Var}\left[\operatorname{Ron}_{t}\right], \mathbf{x}_{\text {hold }}=\mathbf{x}_{C t P t}$ 

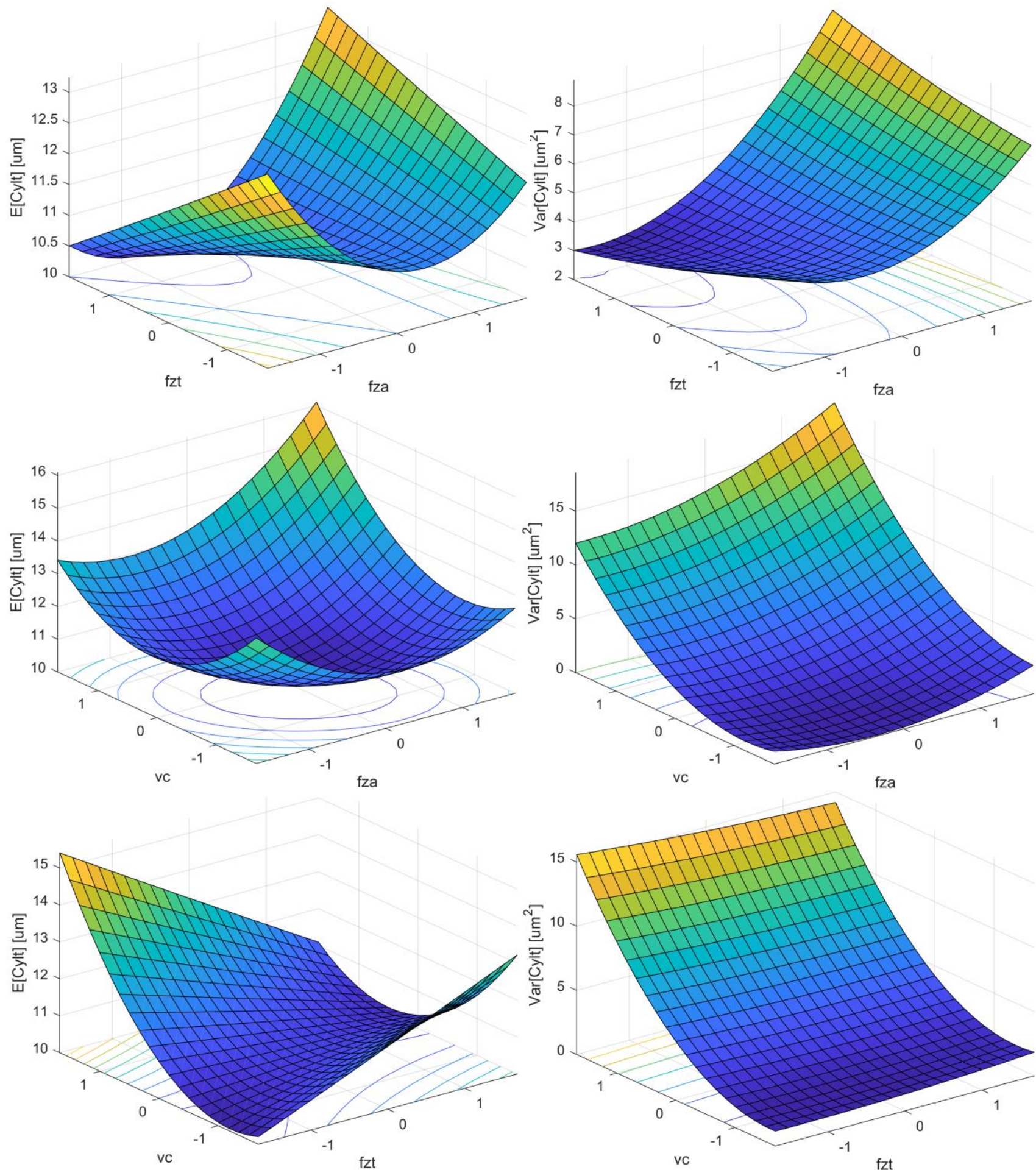

Figure 16. Response surfaces for $E\left[C y l_{t}\right]$ and $\operatorname{Var}\left[C y l_{t}\right], \mathbf{x}_{h o l d}=\mathbf{x}_{C t P t}$

As explained in section 7.1, about similarity analysis, the optimization was performed considering $\operatorname{Ron}_{t}$ to represent the outcomes with regard to geometrical error, since this output is the most frequently used to represent the geometrical error of boreholes and presented best fitting in the modelling. The modelling of $C y l_{t}$ is important to present models which could represent the 
cylindrical error of the borehole, however, its optimization would result in similar solutions to Ron $_{t}$, due to the high correlation between these outcomes.

Table 4 presents the payoff matrix considering the trade-off between $E\left[\operatorname{Ron}_{t}\right]$ and $S\left[\operatorname{Ron}_{t}\right]=$ $\operatorname{Var}\left[\operatorname{Ron}_{t}\right]^{1 / 2}$. The optimal level for the mean was $E\left[\operatorname{Ron}_{t}\right]^{*}=7.08 \mu \mathrm{m}$ and the pseudo-nadir for the mean was $E\left[\operatorname{Ron}_{t}\right]^{\mathrm{N}}=7.42 \mu \mathrm{m}$ reflecting the good quality of the results considering previous literature. There are studies of helical milling in difficult-to-cut materials which could serve for comparison aims. It is important to emphasise that in the present work it was obtained boreholes with $D_{b}=18 \mathrm{~mm}$ and, in general, the geometrical error is proportional to the diameter. In the present approach, it was considered the variance with regard to the noise factors propagated on the response in function of control factors. Then, associated with $E\left[R_{0} n_{t}\right]^{*}=7.08 \mu \mathrm{m}$, the standard deviation $S\left[\operatorname{Ron}_{t}\right]^{\mathrm{N}}$ was $1.321 \mu \mathrm{m}$, the worst solution for $S\left[\operatorname{Ron}_{t}\right]$, while for $E\left[\operatorname{Ron}_{t}\right]^{\mathrm{N}}=7.42 \mu \mathrm{m}$ the standard deviation was $S\left[\text { Ron }_{t}\right]^{*}=1.139 \mu \mathrm{m}$.

Table 4. Payoff matrix for $E\left[\operatorname{Ron}_{t}\right] \times S\left[\right.$ Ron $\left._{t}\right]$

\begin{tabular}{|c|c|}
\hline$E\left[\operatorname{Ron}_{t}\right]$ & 7.081 \\
\hline$S\left[\operatorname{Ron}_{t}\right]$ & 1.321 \\
\hline
\end{tabular}

Figure 17 presents the Pareto frontier with $n_{s u b}=51$ solutions for the dual optimization of $E\left[\operatorname{Ron}_{t}\right]$ and $\operatorname{Var}\left[\operatorname{Ron}_{t}\right]$ through NNC method. No dominated solutions were found in filtering. Some of the optimization results are summarized in Table 5. These results offer some possibilities for the engineer to balance the trade-off between the mean and variance of $\operatorname{Ron}_{t}$. The extreme points of the frontier, called anchor points, are the columns of the payoff matrix in Table 4. The optimal control factors' levels varied in the following intervals $f_{z a} \sim[0.117 ; 0.148] \mu \mathrm{m} /$ tooth, $f_{z t} \sim[0.227 ; 0.233]$ $\mathrm{mm} /$ tooth and $v_{c} \sim[64.0 ; 65.2] \mathrm{m} / \mathrm{min}$. For the optimal level of $S\left[\right.$ Ron $\left._{t}\right]$, i.e., $S\left[\text { Ron }_{t}\right]^{*}=1.139 \mu \mathrm{m}$, the optimal control factor levels were $\mathbf{x}_{\left\{\mathrm{S}[\text { Ront }]^{*}\right\}}=[0.117 \mu \mathrm{m} /$ tooth; $0.227 \mu \mathrm{m} /$ tooth; $64.030 \mathrm{~m} / \mathrm{min}]$. These levels minimize the variability propagated from noise factors in $R_{0} n_{t}$, guaranteeing robustness of geometrical error with regard to tool overhang, hardness and borehole height in the helical milling of AISI H13 hardened steel. 


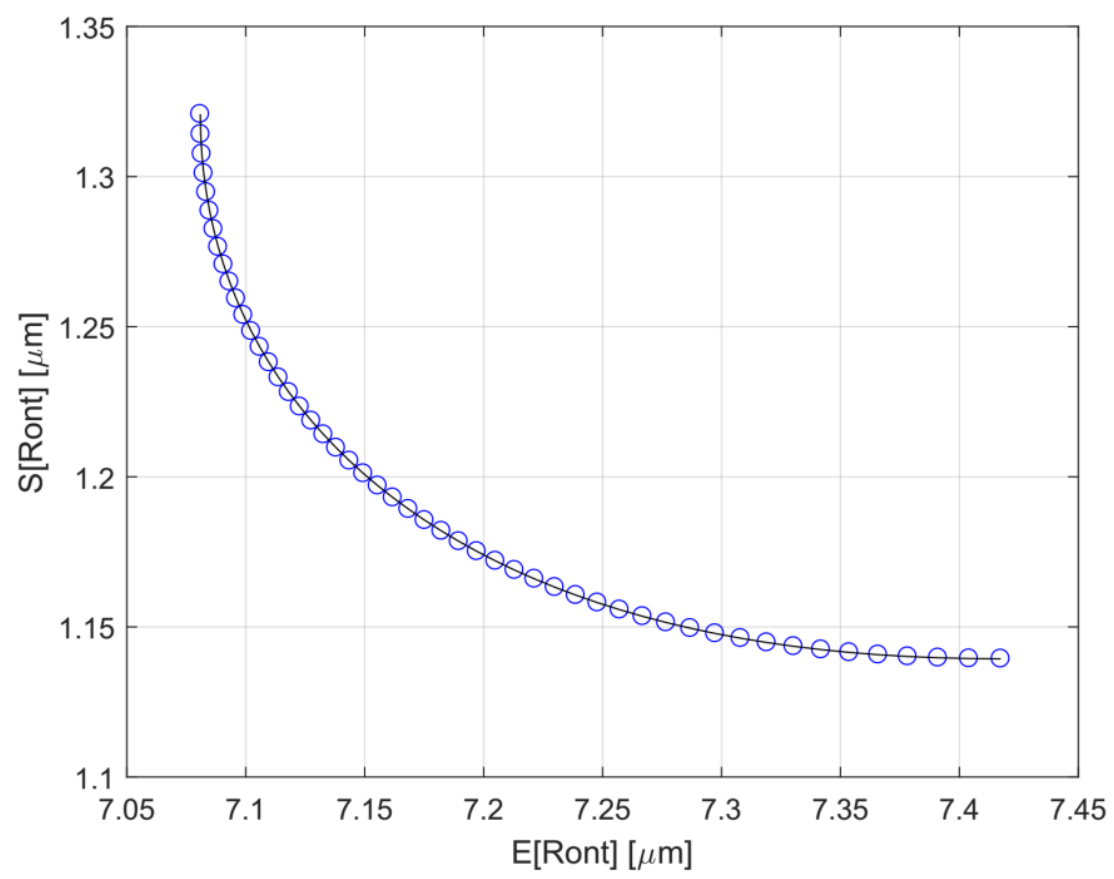

Figure 17. Pareto frontier for $E\left[\operatorname{Ron}_{t}\right]$ and $S\left[\operatorname{Ron}_{t}\right]$

Table 5. NNC optimization results for $E\left[\operatorname{Ron}_{t}\right]$ and $S\left[\operatorname{Ron}_{t}\right]$

\begin{tabular}{ccccccccccc}
\hline & \multicolumn{3}{c}{ Coded } & \multicolumn{3}{c}{ decoded } & \multicolumn{3}{c}{ Responses } \\
\cline { 2 - 9 } $\mathrm{w}_{1}$ & $f_{z a}$ & $f_{z t}$ & $v_{c}$ & $f_{z a}$ & $f_{z t}$ & $v_{c}$ & $E\left(\operatorname{Ron}_{t}\right)$ & $\operatorname{Var}\left(\operatorname{Ron}_{t}\right)$ & $S\left(\right.$ Ron $\left._{t}\right)$ \\
\cline { 5 - 10 } & & & & $\mu \mathrm{m} /$ tooth & $\mathrm{mm} /$ tooth & $\mathrm{m} / \mathrm{min}$ & $\mu \mathrm{m}$ & $\mu \mathrm{m}^{2}$ & $\mu \mathrm{m}$ \\
\hline 0 & -0.660 & 1.534 & 0.201 & 0.117 & 0.227 & 64.030 & 7.417 & 1.298 & 1.139 \\
0.1 & -0.596 & 1.553 & 0.248 & 0.120 & 0.228 & 64.967 & 7.354 & 1.303 & 1.141 \\
0.2 & -0.532 & 1.572 & 0.273 & 0.123 & 0.229 & 65.456 & 7.297 & 1.317 & 1.148 \\
0.3 & -0.470 & 1.589 & 0.285 & 0.127 & 0.229 & 65.706 & 7.248 & 1.341 & 1.158 \\
0.4 & -0.408 & 1.605 & 0.291 & 0.130 & 0.230 & 65.817 & 7.205 & 1.374 & 1.172 \\
0.5 & -0.347 & 1.620 & 0.292 & 0.133 & 0.231 & 65.839 & 7.168 & 1.414 & 1.189 \\
0.6 & -0.286 & 1.632 & 0.290 & 0.136 & 0.232 & 65.798 & 7.138 & 1.463 & 1.210 \\
0.7 & -0.224 & 1.642 & 0.285 & 0.139 & 0.232 & 65.707 & 7.114 & 1.520 & 1.233 \\
0.8 & -0.161 & 1.651 & 0.279 & 0.142 & 0.233 & 65.577 & 7.096 & 1.586 & 1.259 \\
0.9 & -0.097 & 1.657 & 0.271 & 0.145 & 0.233 & 65.410 & 7.085 & 1.660 & 1.289 \\
1 & -0.031 & 1.661 & 0.260 & 0.148 & 0.233 & 65.208 & 7.081 & 1.745 & 1.321 \\
\hline
\end{tabular}

The optimisation of mean square error for Ron $_{t}$, according to the approach in the Equation 15, was also carried out to minimize its bias and variance simultaneously. The MSE[Ron $]$ response 
surface plotted under different perspectives are in Figure 18. The optimization results, presented in Table 6, were similar to dual optimization of $E\left[\operatorname{Ron}_{t}\right]$ and $\operatorname{Var}\left[\operatorname{Ron}_{t}\right]$ with weight $w_{1}=0.8$.
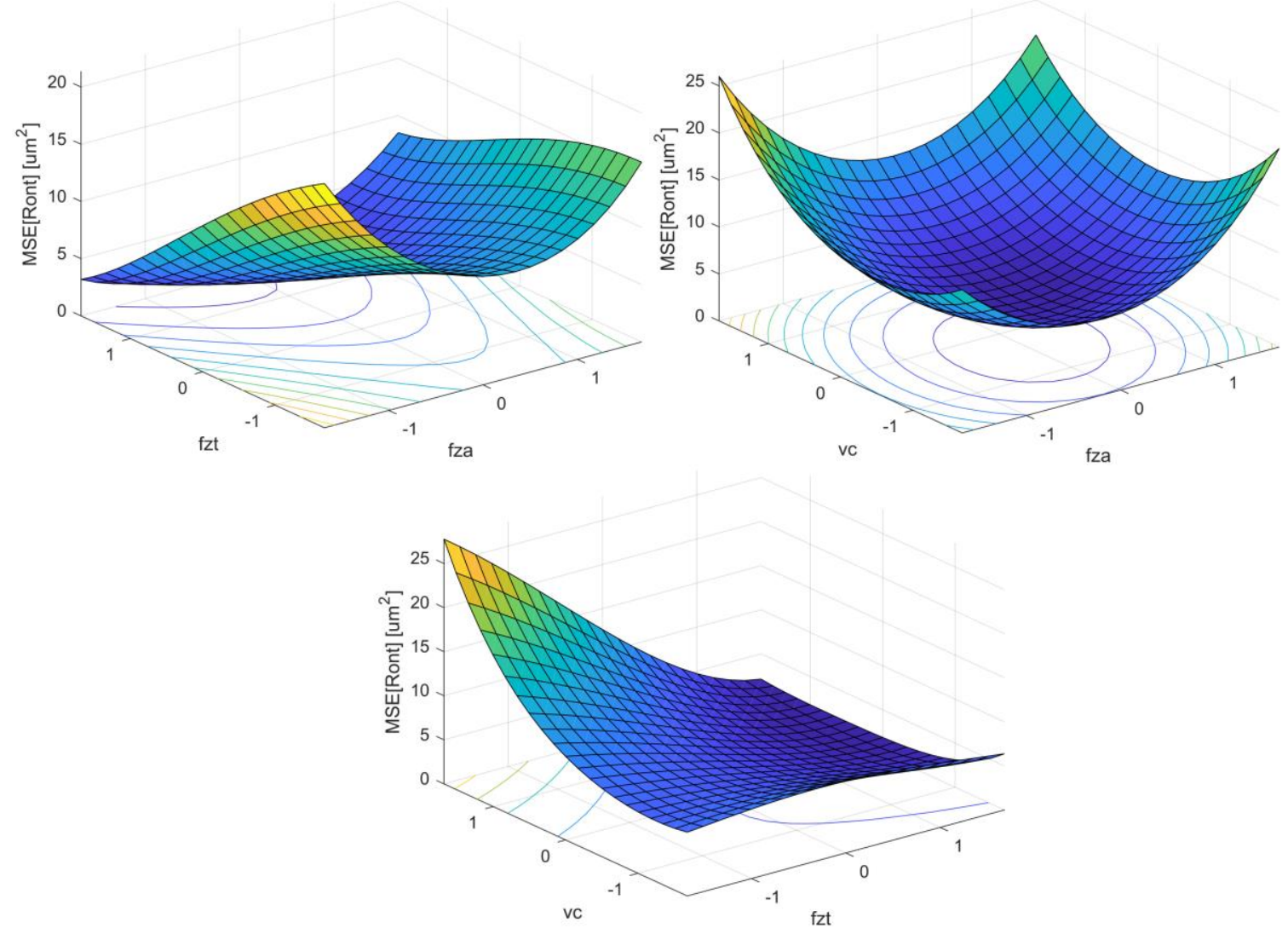

Figure 18. Response surface plots for $M S E_{\text {Ront }}, \mathbf{x}_{\text {hold }}=\mathbf{x}_{C t P t}$

Table 6. $M S E_{\text {Ront }}$ optimization

\begin{tabular}{cccccccccc}
\hline & coded & \multicolumn{4}{c}{ Decoded } & \multicolumn{3}{c}{ Responses } \\
\hline \multirow{2}{*}{$f_{z a}$} & \multirow{2}{*}{$f_{z t}$} & \multirow{2}{*}{$v_{c}$} & $f_{z a}$ & $f_{z t}$ & $v_{c}$ & $E\left(\right.$ Ron $\left._{t}\right)$ & $\operatorname{Var}\left(\right.$ Ron $\left._{t}\right)$ & $S\left(\right.$ Ron $\left._{t}\right)$ & MSE $_{\text {Ront }}$ \\
\cline { 4 - 10 } & & & $\mu \mathrm{m} /$ dente & $\mathrm{mm} /$ dente & $\mathrm{m} / \mathrm{min}$ & $\mu \mathrm{m}$ & $\mu \mathrm{m}^{2}$ & $\mu \mathrm{m}$ & $\mu \mathrm{m}^{2}$ \\
\hline-0.104 & 1.657 & 0.271 & 0.145 & 0.233 & 65.43 & 7.086 & 1.653 & 1.286 & 1.675 \\
\hline
\end{tabular}

\subsection{Roughness on helical milling of AISI H13 hardened steel}

Surface roughness is mostly used as an index to determine the surface finish and, consequently, workpiece quality in machining processes [67-68]. Besides, it is an important requirement to evaluate machined surfaces, affecting lubrication, friction, corrosion resistance, fatigue resistance, and other mechanical properties [69-71]. The average surface roughness, $R_{a}$, and the average maximum height of the profile, $R_{z}$, were evaluated in the borehole surfaces of AISI H13 
hardened steel obtained through helical milling. While the first measures the average of the absolute sampled values of the filtered profile, the second measures the average of the maximum range obtained in each cut-off, aiming to evaluate average and dispersion of the measured profiles.

Figure 19 shows the filtered profiled obtained in one of the measurements realised in the borehole of the workpiece obtained in tests 6 . In Table 2 it can be observed that $l_{b}=-1$, i.e., the measurement position was in the beginning of the borehole.
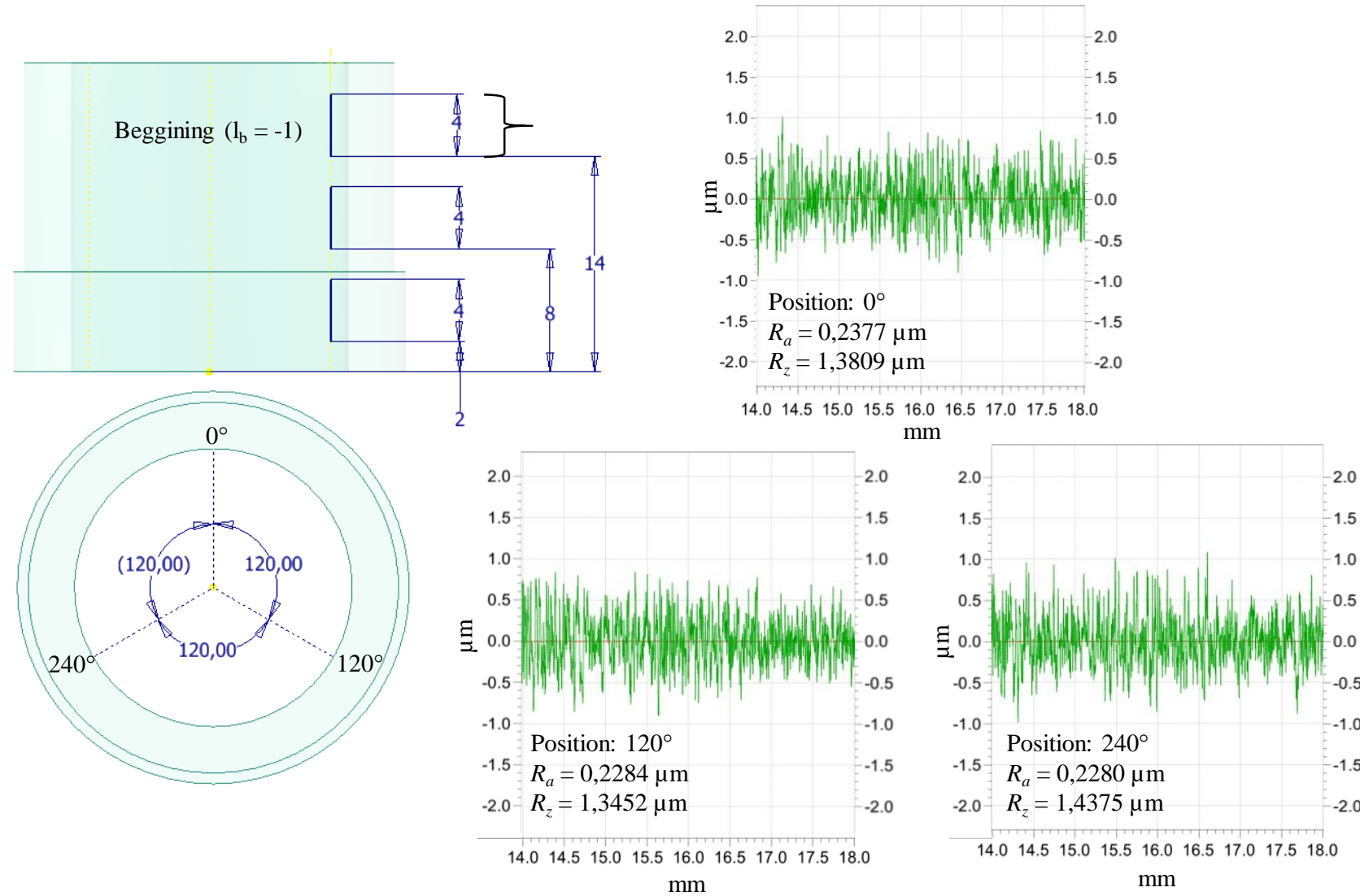

Figure 19. Roughness measurement for test 6

Table 7 presents the results obtained through ANOVA for $R_{a}$ and $R_{z}$. Figures 20 and 21 illustrates the linear, quadratic and interaction effects plot for $R_{a}$ and $R_{z}$, respectively. With regard to $R_{a}$, considering the linear effects, $f_{z t}$ and $v_{c}$ were statistically significant. Without consider the quadratic effect, $f_{z t}$ effect was negative in $R_{a}$. Keeping $f_{z a}$ constant, $f_{z t}$ is inversely proportional to $a_{p}{ }^{*}$ as in Equation 6. As the roughness is measured in the axial direction, $R_{a}$ tends to decrease with decreasing $a_{p}{ }^{*}$. Therefore, the helical milling with more turns for the same borehole depth supports the attainment of a borehole surface with lower roughness. Another point, as explained by Li and Liu [43], if there is a lag between the cutting velocity and the orbital velocity, considering the material removal in the axial direction equals to $a_{p}{ }^{*}$, in a way that at the final of an orbital revolution it has not 
yet finished the chip removal by the edge in action, the peak of the surface left by the frontal cutting edge can be removed by the peripheral cutting edge in action at the next turn. Therefore, it is expected lower roughness in the beginning of the borehole than nearby the exit of the borehole. Cutting velocity, $v_{c}$, presented a positive linear effect in $R_{a}$.

Table 7. ANOVA for $R_{a}$ and $R_{z}$

\begin{tabular}{|c|c|c|c|c|c|}
\hline \multirow{2}{*}{ Source } & $R_{a}$ & $R_{z}$ & \multirow{2}{*}{ Source } & $R_{a}$ & $R_{z}$ \\
\hline & P-valor & P-valor & & P-valor & P-valor \\
\hline Model & 0.000 & 0.000 & Interaction & 0.000 & 0.000 \\
\hline Linear & 0.000 & 0.000 & $f_{z a} \times f_{z t}$ & 0.065 & 0.009 \\
\hline$f_{z a}$ & 0.955 & 0.066 & $f_{z a} \times v_{c}$ & 0.000 & 0.000 \\
\hline$f_{z t}$ & 0.000 & 0.000 & $f_{z t} \times v_{c}$ & 0.000 & 0.000 \\
\hline$v_{c}$ & 0.000 & 0.001 & $f_{z a} \times l_{t o}$ & 0.002 & 0.010 \\
\hline$l_{t o}$ & 0.000 & 0.000 & $f_{z a} \times h_{d}$ & 0.883 & 0.784 \\
\hline$h_{d}$ & 0.000 & 0.000 & $f_{z a} \times l_{b}$ & 0.067 & 0.167 \\
\hline$l_{b}$ & 0.000 & 0.000 & $f_{z t} \times l_{t o}$ & 0.222 & 0.133 \\
\hline Quadratic & 0.068 & 0.082 & $f_{z t} \times h_{d}$ & 0.911 & 0.739 \\
\hline$f_{z a} \times f_{z a}$ & 0.012 & 0.013 & $f_{z t} \times l_{b}$ & 0.027 & 0.044 \\
\hline$f_{z t} \times f_{z t}$ & 0.033 & 0.153 & $v_{c} \times l_{t o}$ & 0.015 & 0.009 \\
\hline$v_{c} \times v_{c}$ & 0.622 & 0.810 & $v_{c} \times h_{d}$ & 0.130 & 0.495 \\
\hline$R^{2}$ & $99.69 \%$ & $98.81 \%$ & $v_{c} \times l_{b}$ & 0.000 & 0.000 \\
\hline$R_{a d j}^{2}$ & $99.45 \%$ & $97.91 \%$ & LOF & 0.377 & 0.400 \\
\hline$R_{\text {prev }}^{2}$ & $94.48 \%$ & $84.81 \%$ & $S$ & 1.023 & 0.986 \\
\hline
\end{tabular}

With regard to quadratic effects, $f_{z a}$ was positive with convexity downward. For $f_{z a}$ varying from low levels until $0.15 \mu \mathrm{m} / \mathrm{rev}$ the $f_{z a}$ increment results in $R_{a}$ growth once $f_{z a}$ is proportional to $a_{p}{ }^{*}$, according to Equation 6. Nonetheless, from $0.15 \mu \mathrm{m} / \mathrm{rev}$ to higher levels of $f_{z a}$ it was observed a decrease in $R_{a}$. The three noise factors were statistically significant in $R_{a}$. The increase of tool overhang effect, $l_{t o}$, entails tool deflection increase and, consequently, higher vibration levels, leading to instabilities during cutting and, possibly, a rougher surface. The effect of the borehole measurement height, $l_{b}$, is also related to the smoothing of the surface for the tangential cutting edges due to the higher orbital rotation levels in the beginning of the borehole, as explained by Li and Liu [43]. With regard to workpiece hardness, $h_{d}$, as it increases, the roughness decreases due to the lower 
deformation of the machined surface. The same behaviour was observed in the helical milling of AISI 4340 hardened steel [18]. The $R_{z}$ results were graphically and statistically similar to $R_{a}$, according to Figures 20 and 21.

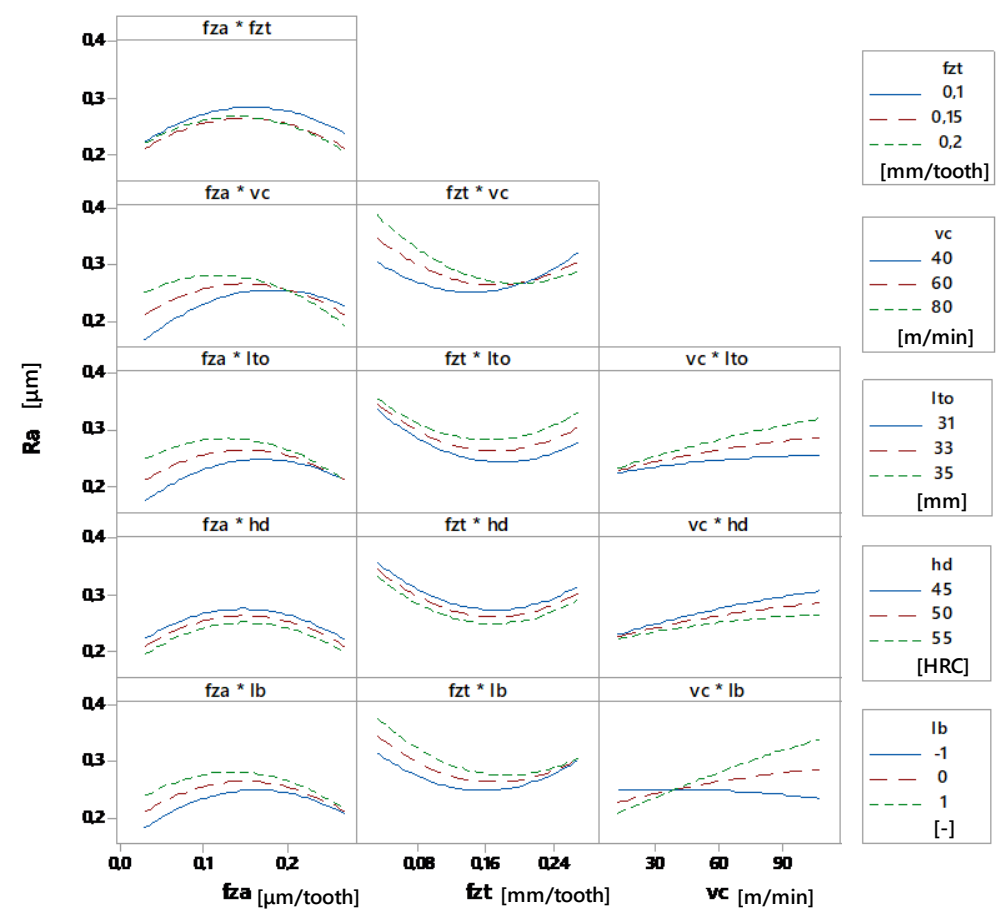

Figure 20. Interaction plots for $R_{a}$

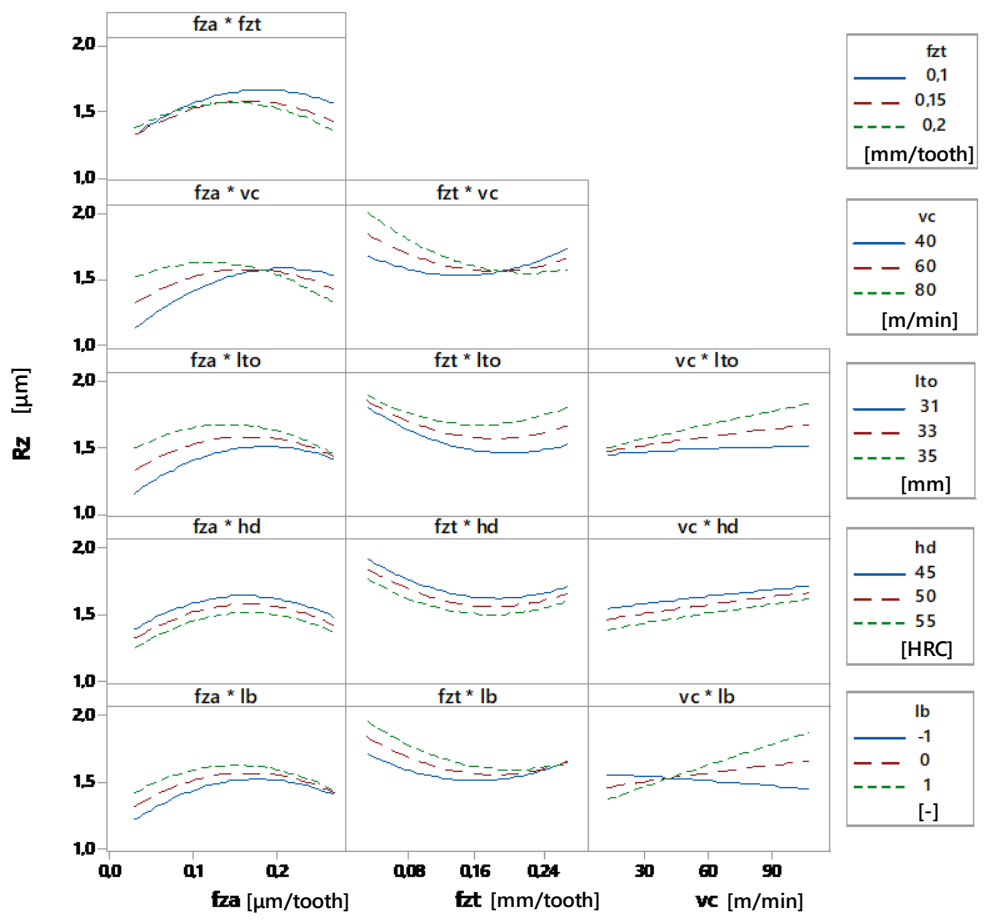

Figure 21. Interaction plots for $R_{z}$ 
With regard to the interactions among process factors, all three combinations were statistically significant for $R_{z}$, while for $R_{a}$ only $f_{z a} \times f_{z t}$ was not significant. The process $\times$ noise interactions $f_{z a} \times l_{t o}, f_{z t} \times l_{b}, v_{c} \times l_{t o}, v_{c} \times l_{b}$ were statistically significant in $R_{a}$ and $R_{z}$. High levels of $f_{z a}$ assure robustness of roughness with regard to $l_{t o}$ variation. Similarly, $f_{z t}$ set in extreme positive levels, considering the experimental space, guarantees the insensitivity of roughness outcomes with regard to $l_{b}$ variation, providing a borehole with lower geometrical error variation in its complete height. As $f_{z t}$ is inversely proportional to $a_{p}{ }^{*}$, as $f_{z t}$ is higher, higher is the number of orbital turns of the tool, enabling the surface smoothing. Lower cutting velocities graphically indicate constant roughness with regard to $l_{t o}$ variation. However, to achieve robustness of roughness with regard to borehole height, $v_{c}$ must be set in $40 \mathrm{~m} / \mathrm{min}$.

Equations 42 and 43 presents the response models for $R_{a}$ and $R_{z}$, respectively, obtained through WLS. The homoscedasticity test results are in the Appendix B. These models presented experimental error estimated through ANOVA respectively equals to $\hat{\sigma}_{R_{a}}=1.023$ and $\hat{\sigma}_{R_{z}}=0.986$. With regard to goodness-of-fit statistics, the models well explain the data variability with $R_{a j}{ }^{2}=$ $99.45 \%$ for $R_{a}$ and $R_{a j}{ }^{2}=97.91 \%$ for $R_{z}$. In addition, the models presented good prediction capacity with $R_{\text {prev }}{ }^{2}=94.48 \%$ and $R_{\text {prev }}{ }^{2}=84.81 \%$ for $R_{a}$ and $R_{z}$, respectively. The LOF tests appointed no evidence to reject the null hypothesis of do not have lack-of-fit of the data, since the reported pvalues were 0.377 and 0.400 , for $R_{a}$ and $R_{z}$. The signal of the coefficients in the response models, which are associated to the effects, can be confirmed through effects plots in Figures 20 and 21.

$$
\begin{aligned}
R_{a}(\mathbf{x}, \mathbf{z}) & =0,2648-0.0001 \times f_{z a}-0,0087 \times f_{z t}+0,0124 \times v_{c}-0,0093 \times f_{z a}{ }^{2}+0,0105 \times f_{z t}{ }^{2} \\
& -0,0013 \times v_{c}{ }^{2}-0,0033 \times f_{z a} \times f_{z t}-0,0123 \times f_{z a} \times v_{c}-0,0118 \times f_{z t} \times v_{c}+0,0184 \times l_{t o} \\
& -0,0123 \times h_{d}+0,0161 \times l_{b}-0,0079 \times f_{z a} \times l_{t o}+0,0004 \times f_{z a} \times h_{d}-0,0051 \times f_{z a} \times l_{b} \\
& +0,0035 \times f_{z t} \times l_{t o}+0,0003 \times f_{z t} \times h_{d}-0,0060 \times f_{z t} \times l_{b}+0,0060 \times v_{c} \times l_{t o} \\
& -0,0035 \times v_{c} \times h_{d}+0,0154 \times v_{c} \times l_{b} \\
R_{z}(\mathbf{x}, \mathbf{z}) & =1,5764+0.0210 \times f_{z a}-0,0397 \times f_{z t}+0,0419 \times v_{c}-0,0355 \times f_{z a}{ }^{2}+0,0310 \times f_{z t}{ }^{2} \\
& -0,0018 \times v_{c}{ }^{2}-0,0279 \times f_{z a} \times f_{z t}-0,0633 \times f_{z a} \times v_{c}-0,0513 \times f_{z t} \times v_{c}+0,0935 \times l_{t o} \\
& -0,0645 \times h_{d}+0,0593 \times l_{b}-0,0326 \times f_{z a} \times l_{t o}+0,0035 \times f_{z a} \times h_{d}-0,0181 \times f_{z a} \times l_{b} \\
& +0,0214 \times f_{z t} \times l_{t o}+0,0047 \times f_{z t} \times h_{d}-0,0266 \times f_{z t} \times l_{b}+0,0284 \times v_{c} \times l_{t o} \\
& +0,0065 \times v_{c} \times h_{d}+0,0656 \times v_{c} \times l_{b}
\end{aligned}
$$


Equations 44 and 45 present the mean and variance models for $R_{a}$, enabling to achieve robustness to this response with regard to noise factors. The response surfaces for these models are plotted in Figure 22. Equations 46 and 47 presents the mean and variance models for $R_{z}$. These models are graphically depicted in Figure 23.

$$
\begin{gathered}
E\left[R_{a}(\mathbf{x}, \mathbf{z})\right]=0,2648-0.0001 \times f_{z a}-0,0087 \times f_{z t}+0,0124 \times v_{c}-0,0093 \times f_{z a}^{2}+0,0105 \times f_{z t}{ }^{2} \\
-0,0013 \times v_{c}{ }^{2}-0,0033 \times f_{z a} \times f_{z t}-0,0123 \times f_{z a} \times v_{c}-0,0118 \times f_{z t} \times v_{c} \\
\operatorname{Var}\left[R_{a}(\mathbf{x}, \mathbf{z})\right]=1,0467-0.0005 \times f_{z a}-0,0001 \times f_{z t}+0,0008 \times v_{c}+0,0001 \times f_{z a}{ }^{2}+0,00005 \times f_{z t}{ }^{2} \\
+0,0003 \times v_{c}{ }^{2}+0,00001 \times f_{z a} \times f_{z t}-0,0003 \times f_{z a} \times v_{c}-0,0001 \times f_{z t} \times v_{c} \\
\begin{array}{c}
E\left[R_{z}(\mathbf{x}, \mathbf{z})\right]=1,5764+0.0210 \times f_{z a}-0,0397 \times f_{z t}+0,0419 \times v_{c}-0,0355 \times f_{z a}{ }^{2}+0,0310 \times f_{z t}{ }^{2} \\
-0,0018 \times v_{c}{ }^{2}-0,0279 \times f_{z a} \times f_{z t}-0,0633 \times f_{z a} \times v_{c}-0,0513 \times f_{z t} \times v_{c} \\
\operatorname{Var}\left[R_{z}(\mathbf{x}, \mathbf{z})\right]=1,1064-0.0087 \times f_{z a}+0,0003 \times f_{z t}+0,0122 \times v_{c}+0,0014 \times f_{z a}{ }^{2}+0,0012 \times f_{z t}{ }^{2} \\
+0,0052 \times v_{c}{ }^{2}-0,0004 \times f_{z a} \times f_{z t}-0,0042 \times f_{z a} \times v_{c}-0,0022 \times f_{z t} \times v_{c}
\end{array}
\end{gathered}
$$



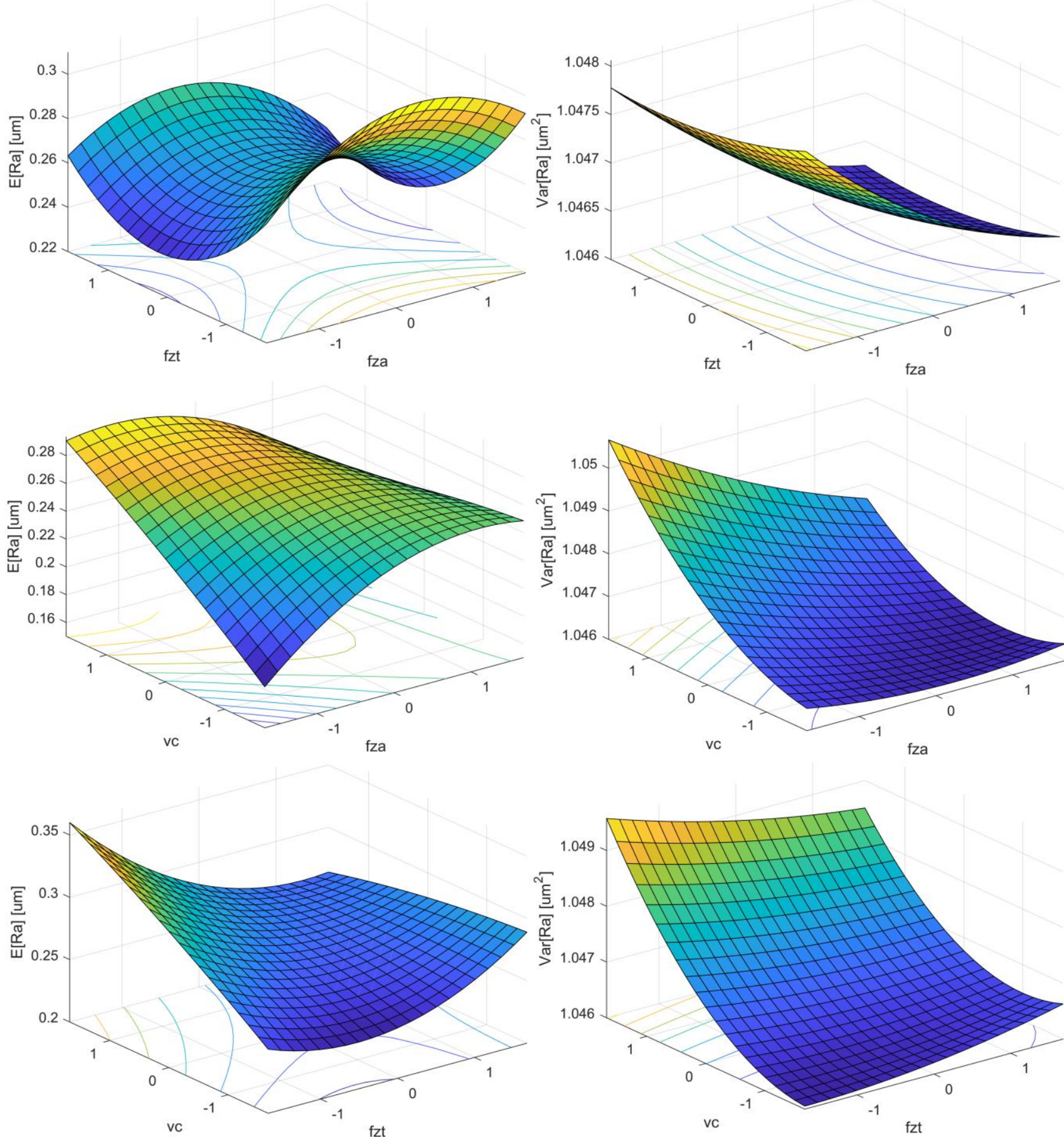

Figure 22. Response surfaces for $E\left[R_{a}\right] \times \operatorname{Var}\left[R_{a}\right], \mathbf{x}_{h o l d}=\mathbf{x}_{C t P t}$ 

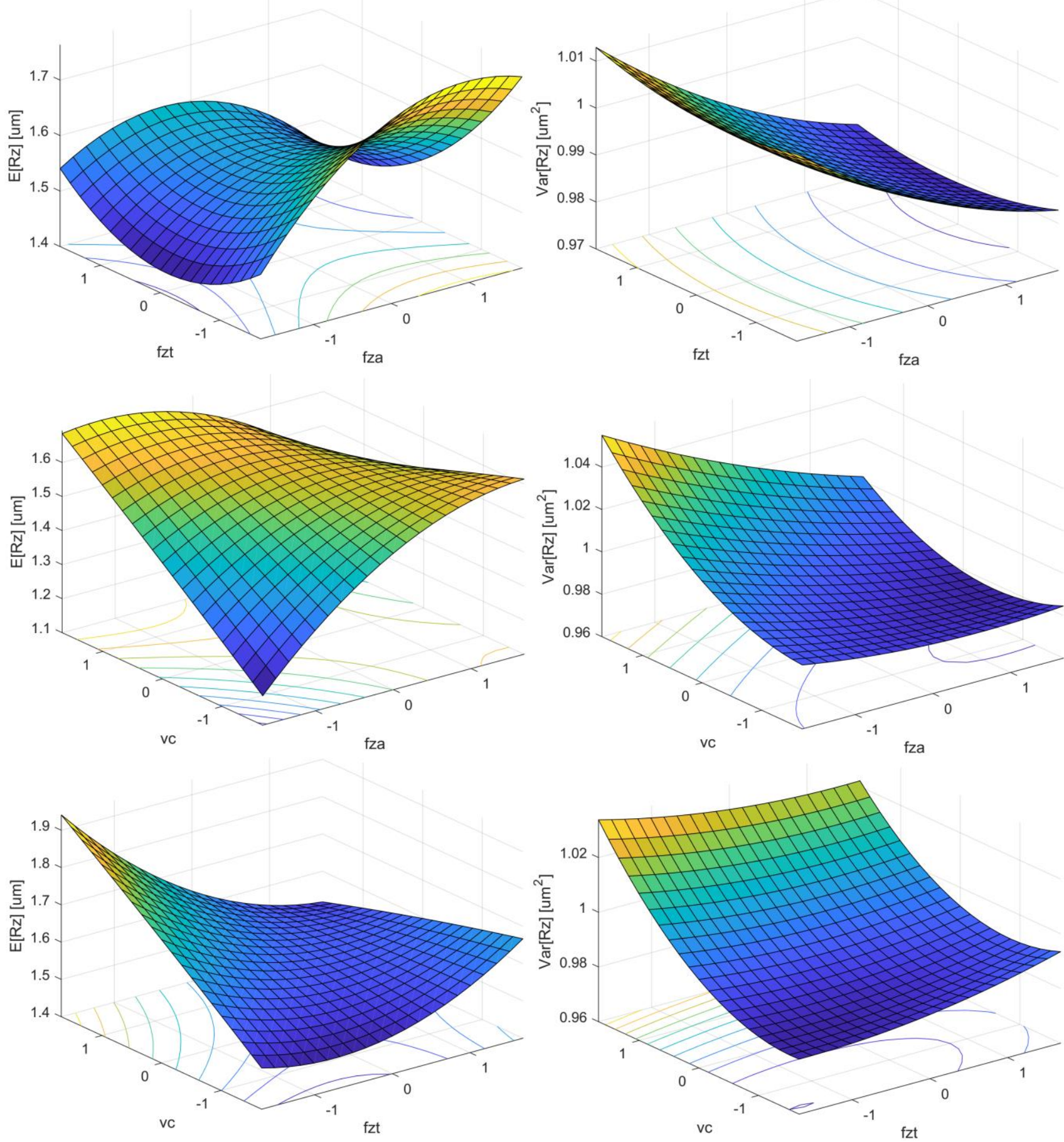

Figure 23. Response surfaces for $E\left[R_{z}\right] \times \operatorname{Var}\left[R_{z}\right], \mathbf{x}_{h o l d}=\mathbf{x}_{C t P t}$

The optimization of $R_{a}$ was performed considering the similarity analysis in section 7.1. $R_{a}$ is the most important roughness parameter and presented best fitting. The optimization of $R_{z}$ would result in analogous results to $R_{a}$ due to the high positive correlation between these outcomes.

Considering the individual optimization of $E\left[R_{a}\right]$ and $S\left[R_{a}\right]$ the payoff matrix is presented in Table 8 . The payoff matrix summarizes the limit values for $E\left[R_{a}\right]$ and $S\left[R_{a}\right]$ in bi-objective 
optimization. Then, $E\left[R_{a}\right]$ varies from $E\left[R_{a}\right]^{*}=0.22 \mu \mathrm{m}$ to $E\left[R_{a}\right]^{\mathrm{N}}=0.26 \mu \mathrm{m}$, while $S\left[R_{a}\right]$ varies from $S\left[R_{a}\right]^{*}=1.0228 \mu \mathrm{m}$ to $S\left[R_{a}\right]^{\mathrm{N}}=1.0230 \mu \mathrm{m}$. To explore the trade-off between $E\left[R_{a}\right]$ and $S\left[R_{a}\right]$ the NNC multi-objective optimization method was applied. The results for $n_{\text {sub }}=51$ sub-problems are plotted in Figure 24. In this case, it can be observed that no dominated solutions were found in filtering. For 11 most representative trade-off points, the results for NNC bi-objective optimization are summarized in Table 9. These results are options to manage the trade-off between mean and variance of roughness. When the engineer is interested in lower levels of the mean of roughness he should assign a higher level of $w_{1}$ which is the weight related to $E\left[R_{a}\right]$, being aware of the loss in variance, since its weight is $w_{2}=1-w_{1}$. On the contrary, when the engineer wants to achieve a more promising robustness scenario for borehole surface roughness, with regard to tool overhang, workpiece hardness and borehole height variation, is recommended to the engineer select a Pareto optimal solution which prioritises $\operatorname{Var}\left[R_{a}\right]$, with $w_{2}$ higher than $w_{1}$.

Table 8. Payoff matrix for $E\left[R_{a}\right] \times S\left[R_{a}\right]$

\begin{tabular}{lcc}
\hline$E\left[R_{a}\right]$ & $\mathbf{0 . 2 2}$ & 0.26 \\
$S\left[R_{a}\right]$ & 1.0230 & $\mathbf{1 . 0 2 2 8}$ \\
\hline
\end{tabular}

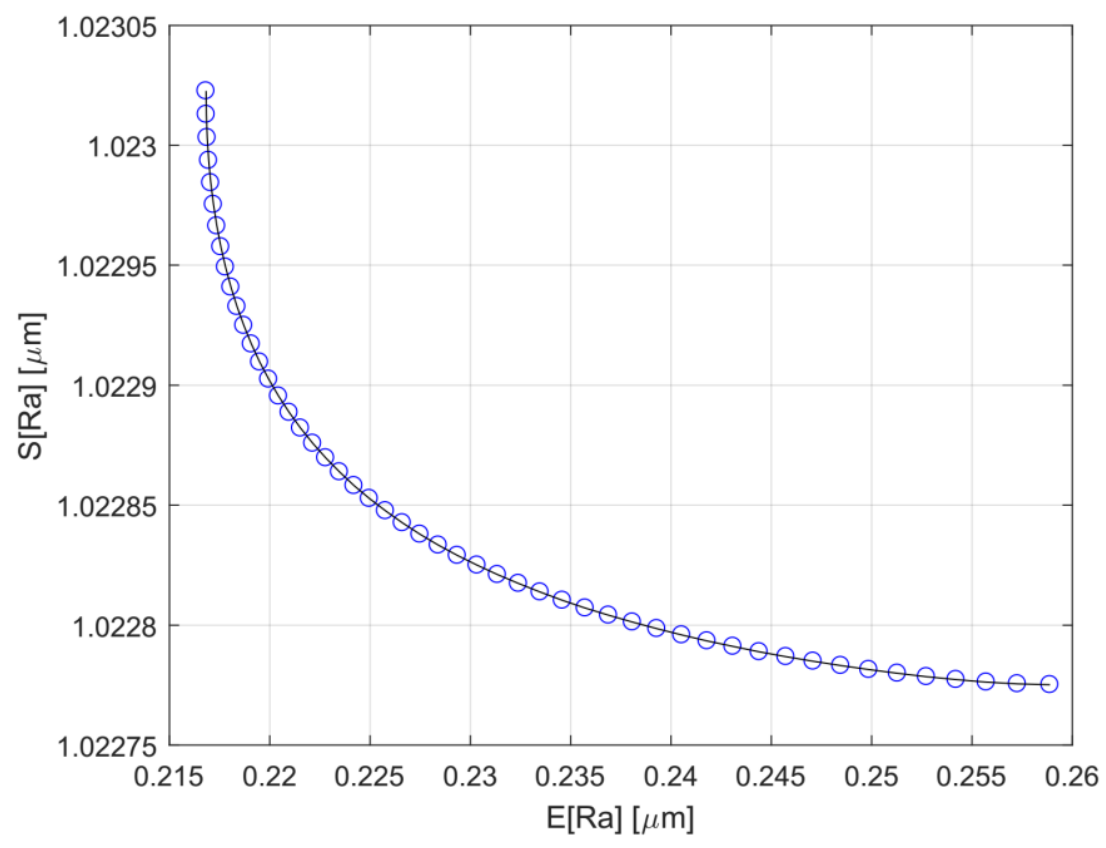

Figure 24. Pareto frontier for $E\left[R_{a}\right]$ and $S\left[R_{a}\right]$ 
Table 9. NNC optimization results for $E\left[R_{a}\right]$ and $\operatorname{Var}\left[R_{a}\right]$

\begin{tabular}{cccccccccc}
\hline & \multicolumn{3}{c}{ coded } & \multicolumn{3}{c}{ decoded } & \multicolumn{3}{c}{ Responses } \\
\cline { 2 - 9 } $\mathrm{w}_{1}$ & \multirow{2}{*}{$f_{z a}$} & $f_{z t}$ & $v_{c}$ & $f_{z a}$ & $f_{z t}$ & $v_{c}$ & $E\left(R_{a}\right)$ & $\operatorname{Var}\left(R_{a}\right)$ & $S\left(R_{a}\right)$ \\
\cline { 5 - 10 } & & & & $\mu \mathrm{m} /$ dente & $\mathrm{mm} / \mathrm{dente}$ & $\mathrm{m} / \mathrm{min}$ & $\mu \mathrm{m}$ & $\mu \mathrm{m}^{2}$ & $\mu \mathrm{m}$ \\
\hline 0.000 & 0.777 & -0.864 & -1.216 & 0.189 & 0.107 & 35.682 & 0.259 & 1.0461 & 1.0228 \\
0.100 & 0.373 & -0.821 & -1.420 & 0.169 & 0.109 & 31.608 & 0.251 & 1.0461 & 1.0228 \\
0.200 & 0.102 & -0.745 & -1.504 & 0.155 & 0.113 & 29.911 & 0.244 & 1.0461 & 1.0228 \\
0.300 & -0.124 & -0.649 & -1.547 & 0.144 & 0.118 & 29.069 & 0.238 & 1.0461 & 1.0228 \\
0.400 & -0.331 & -0.545 & -1.556 & 0.133 & 0.123 & 28.878 & 0.232 & 1.0462 & 1.0228 \\
0.500 & -0.527 & -0.445 & -1.534 & 0.124 & 0.128 & 29.321 & 0.227 & 1.0462 & 1.0228 \\
0.600 & -0.711 & -0.358 & -1.482 & 0.114 & 0.132 & 30.368 & 0.223 & 1.0463 & 1.0229 \\
0.700 & -0.878 & -0.290 & -1.405 & 0.106 & 0.136 & 31.900 & 0.220 & 1.0463 & 1.0229 \\
0.800 & -1.025 & -0.238 & -1.312 & 0.099 & 0.138 & 33.761 & 0.218 & 1.0464 & 1.0229 \\
0.900 & -1.152 & -0.199 & -1.209 & 0.092 & 0.140 & 35.825 & 0.217 & 1.0465 & 1.0230 \\
1.000 & -1.262 & -0.167 & -1.100 & 0.087 & 0.142 & 38.009 & 0.217 & 1.0466 & 1.0230 \\
\hline
\end{tabular}

As an approach to minimize bias and variance, the $\operatorname{MSE}\left[R_{a}\right]$ was also optimized. Figure 25 shows the response surface plot for $\operatorname{MSE}\left[R_{a}\right]$ in different perspectives. The optimization results of $\operatorname{MSE}\left[R_{a}\right]$ are summarized in Table 10 . These results were similar to the ones obtained with $w_{1}=0.9$ and $w_{1}=1$ in NNC for $E\left[R_{a}\right]$ and $\operatorname{Var}\left[R_{a}\right]$ as can be confirmed in Table 9.

Table 10. $M S E_{R a}$ optimization

\begin{tabular}{ccccccccccc}
\hline & coded & \multicolumn{4}{c}{ uncoded } & \multicolumn{5}{c}{ responses } \\
\hline \multirow{2}{*}{$f_{z a}$} & \multirow{2}{*}{$f_{z t}$} & \multirow{2}{*}{$v_{c}$} & $f_{z a}$ & $f_{z t}$ & $v_{c}$ & $E(R a)$ & $\operatorname{Var}(R a)$ & $S(R a)$ & $M S E R a$ \\
\cline { 4 - 10 } & & & $\mu \mathrm{m} /$ tooth & $\mathrm{mm} /$ tooth & $\mathrm{m} / \mathrm{min}$ & $\mu \mathrm{m}$ & $\mu \mathrm{m}^{2}$ & $\mu \mathrm{m}$ & $\mu \mathrm{m}^{2}$ \\
\hline-1.202 & -0.184 & -1.162 & 0.090 & 0.141 & 36.8 & 0.217 & 1.047 & 1.023 & 1.047 \\
\hline
\end{tabular}



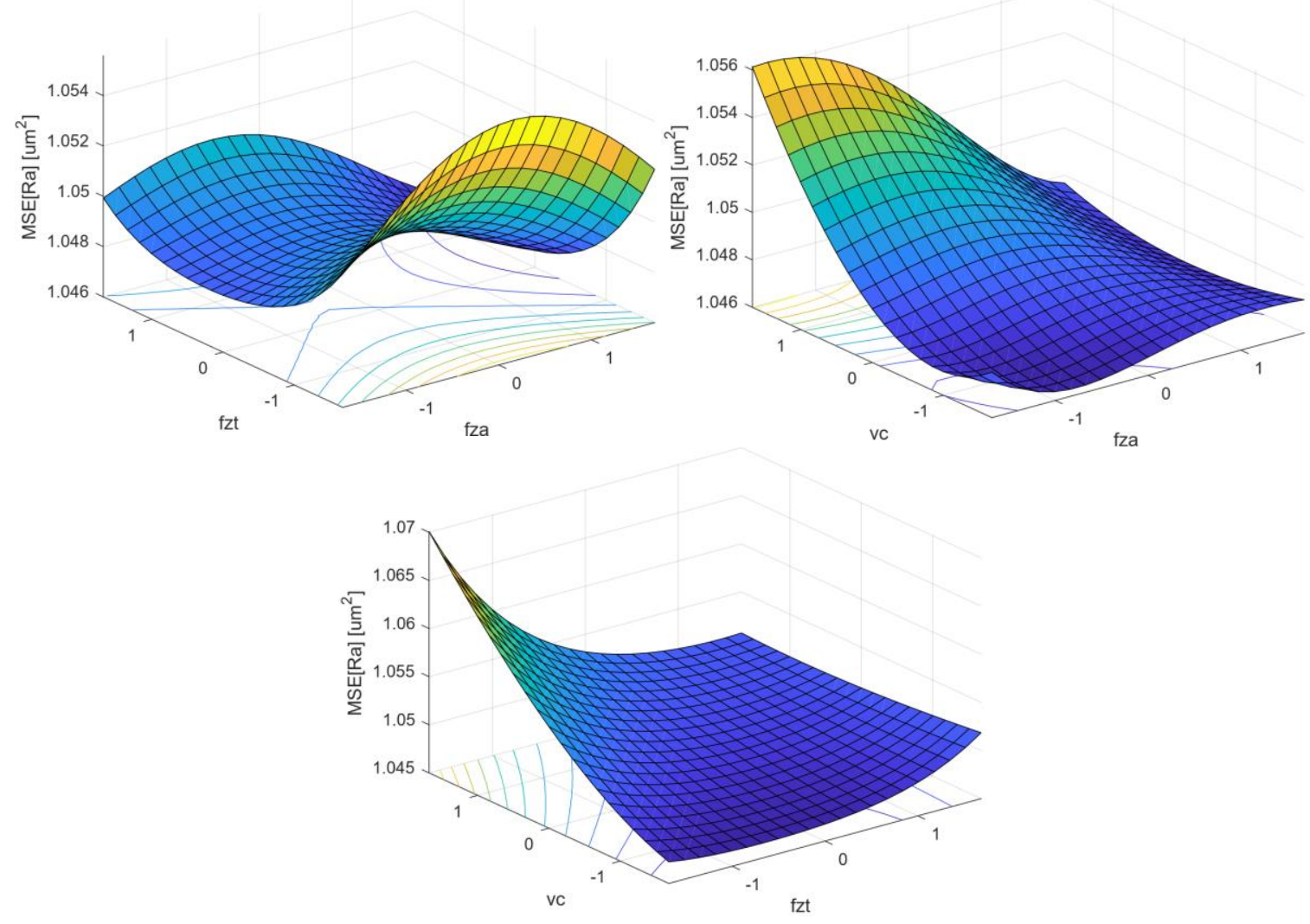

Figure 25. Response surface plots for $M S E_{R a}, \mathbf{x}_{h o l d}=\mathbf{x}_{C t P t}$

\subsection{Bi-objective optimization of $M S E\left[R_{a}\right]$ and $M S E\left[R_{0} n_{t}\right]$}

With the purpose of evaluating the trade-off between roughness and roundness, the biobjective optimization between $M S E\left[R_{0} n_{t}\right]$ and $M S E\left[R_{a}\right]$ was performed. Table 11 presents the payoff matrix showing the trade-off limits, i.e., the anchor points obtained by individual optimization. Specially in $M S E\left[R_{0} n_{t}\right]$ there are an expressive difference between the utopia and pseudo-nadir points. The Pareto frontier, with $n_{s u b}=51$ non-dominated solutions, is plotted in Figure 26(a). For the sake of simplicity, only 11 Pareto optimal solutions are summarised in Table 12. Figure 26(b) shows the design space with the contour plots for $M S E\left[\operatorname{Ron}_{t}\right]$ and $M S E\left[R_{a}\right]$, the design points and the solutions in the design space, denoted by $\mathbf{x}^{*}$.

Table 11. Payoff matrix for $E\left[R_{a}\right] \times S\left[R_{a}\right]$

\begin{tabular}{lll}
\hline$E\left[M S E_{R a}\right]$ & $\mathbf{1 . 3 6 4}$ & 8.301 \\
$S\left[\mathrm{MSE}_{\text {Ront }}\right]$ & 1.051 & $\mathbf{1 . 0 4 6}$
\end{tabular}


To achieve low roughness levels it is recommended to set up the uncoded-Pareto optimal solution with uncoded optimal vector' levels $\mathbf{x}_{[w 1=0]}^{*}=[0.116 \mu \mathrm{m} /$ tooth; $0.131 \mathrm{~mm} /$ tooth; 30.08 $\mathrm{m} / \mathrm{min}]^{\mathrm{T}}$, this solution reported the optimal level for $M S E\left[R_{a}\right]$ and, consequently, lower $E\left[R_{a}\right]$ levels, as exposed in Table 12. As can be observed in the Figure 26(b), this red star point with coded levels $\mathbf{x}_{[w 1=0]}^{*}=[-0.670 ;-0.376 ;-1.496]^{\mathrm{T}}$, is a constrained Pareto optimal point, near the contour plots minimum of $M S E\left[R_{a}\right]$ graphically depicted with continuous green lines, since it is near the spherical region which could be formed considering the design points denoted by magenta diamonds. To reach low geometrical error the optimal vector $\mathbf{x}_{[w 1=1]}{ }=[0.124 \mu \mathrm{m} / \text { tooth; } 0.229 \mathrm{~mm} / \text { tooth; } 65.53 \mathrm{~m} / \mathrm{min}]^{\mathrm{T}}$, which is the optimal design point related to the optimal solution which prioritizes $M S E\left[\operatorname{Ron}_{t}\right]$, responsible for the lower level of $E\left[\operatorname{Ron}_{t}\right]$, as observed in the bottom line of the Table 12. This solution, with coded levels $\mathbf{x}_{[w 1=0]}^{*}=[-0.519 ; 1.576 ; 0.276]^{\mathrm{T}}$, is near the centre of the contour “ellipses" for $M S E\left[R o n_{t}\right]$, graphically depicted with blue dashed lines. This point is also a constrained Pareto optimal solution, although it is not possible to conclude graphically, due to the $f_{z t}{ }^{*}$ value which uncoded was equal to 1.576. Taking the square root of the sum of squares of the coded levels of process factors, it can be concluded that the result is equal to the squared spherical radius equals to $\left(2^{3}\right)^{1 / 2}$. The solutions in the design space between the anchor points solutions are the most part not constrained Pareto optimal solutions. Only some of the solutions nearby the $M S E\left[R_{a}\right]$ minima are in the experimental region limit.
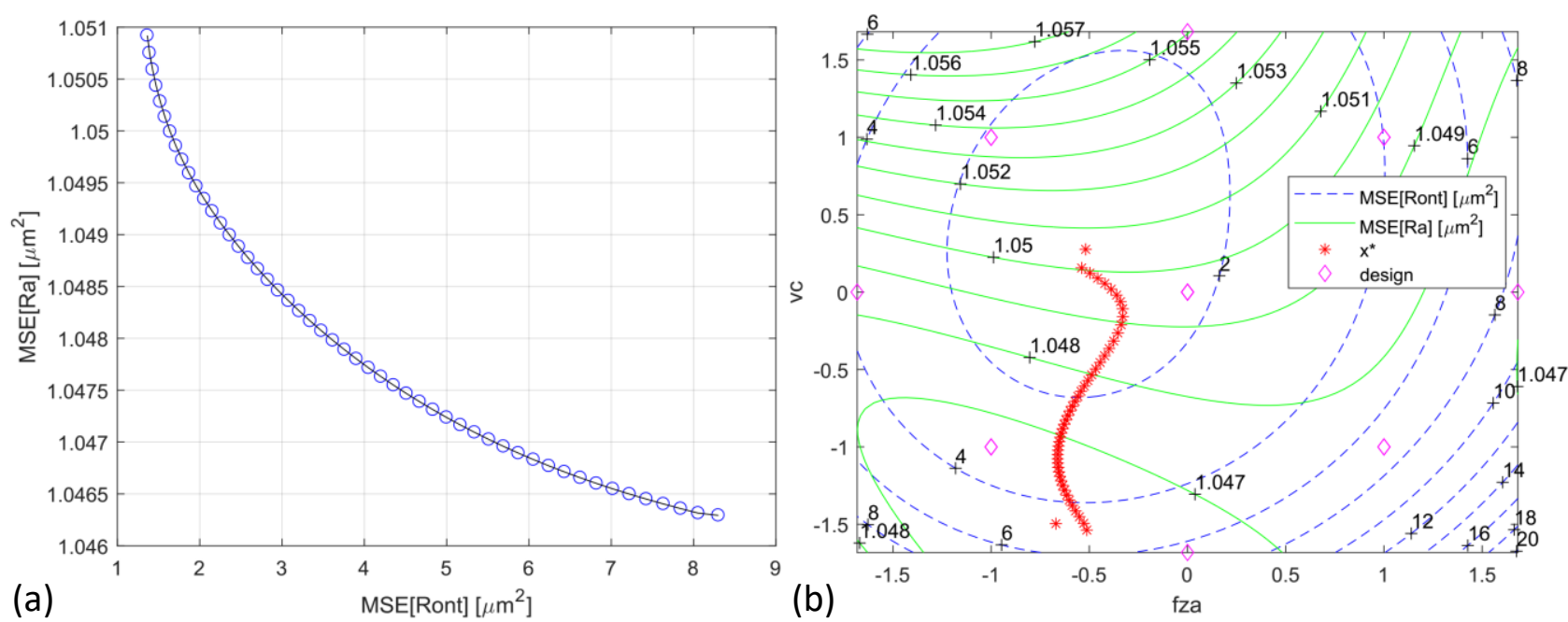

Figure 26. (a) Pareto frontier; (b) Design space for $f_{z a}$ and $v_{c}$. Contour plots with $f_{z t}{ }^{*}$ MSERont $]=0.229$ $\mu \mathrm{m} /$ tooth and $f_{z t}{ }^{*}{ }^{*}$ MSERa] $=0.131 /$ tooth 
Table 12. NNC optimization results for $M S E\left[\operatorname{Ron}_{t}\right]$ and $M S E\left[R_{a}\right]$

\begin{tabular}{|c|c|c|c|c|c|c|c|c|c|c|c|c|c|}
\hline \multirow{3}{*}{$\mathrm{w}_{1}$} & \multicolumn{3}{|c|}{ coded } & \multicolumn{3}{|c|}{ decoded } & \multicolumn{2}{|c|}{ Responses } & \multirow{3}{*}{$\frac{M S E(\text { Ront })}{\mu \mathrm{m}^{2}}$} & \multirow{3}{*}{$\begin{array}{c}E\left(R_{a}\right) \\
\mu \mathrm{m}\end{array}$} & \multirow{3}{*}{$\begin{array}{c}S\left(R_{a}\right) \\
\mu \mathrm{m}\end{array}$} & \multirow{3}{*}{$\frac{M S E(R a)}{\mu \mathrm{m}^{2}}$} & \multirow{3}{*}{$d_{j+}$} \\
\hline & \multirow[b]{2}{*}{$f_{z a}$} & \multirow[b]{2}{*}{$f_{z t}$} & \multirow[b]{2}{*}{$v_{c}$} & $f_{z a}$ & $f_{z t}$ & $v_{c}$ & E(Ront) & S(Ront) & & & & & \\
\hline & & & & $\mu \mathrm{m} /$ tooth & $\mathrm{mm} /$ tooth & $\mathrm{m} / \mathrm{min}$ & $\mu \mathrm{m}$ & $\mu \mathrm{m}$ & & & & & \\
\hline 0 & -0.670 & -0.376 & -1.496 & 0.116 & 0.131 & 30.082 & 9.490 & 1.580 & 8.301 & 0.224 & 1.023 & 1.046 & 1.000 \\
\hline 0.1 & -0.587 & -0.058 & -1.378 & 0.121 & 0.147 & 32.445 & 9.365 & 1.415 & 7.221 & 0.232 & 1.023 & 1.046 & 0.845 \\
\hline 0.2 & -0.643 & 0.254 & -1.219 & 0.118 & 0.163 & 35.617 & 9.185 & 1.347 & 6.241 & 0.237 & 1.023 & 1.047 & 0.711 \\
\hline 0.3 & -0.661 & 0.474 & -1.077 & 0.117 & 0.174 & 38.467 & 8.994 & 1.296 & 5.339 & 0.243 & 1.023 & 1.047 & 0.599 \\
\hline 0.4 & -0.648 & 0.645 & -0.939 & 0.118 & 0.182 & 41.230 & 8.797 & 1.251 & 4.512 & 0.248 & 1.023 & 1.047 & 0.520 \\
\hline 0.5 & -0.609 & 0.787 & -0.797 & 0.120 & 0.189 & 44.069 & 8.594 & 1.212 & 3.759 & 0.254 & 1.023 & 1.048 & 0.488 \\
\hline 0.6 & -0.542 & 0.912 & -0.641 & 0.123 & 0.196 & 47.183 & 8.380 & 1.179 & 3.079 & 0.259 & 1.023 & 1.048 & 0.511 \\
\hline 0.7 & -0.445 & 1.032 & -0.454 & 0.128 & 0.202 & 50.911 & 8.147 & 1.157 & 2.474 & 0.264 & 1.023 & 1.049 & 0.582 \\
\hline 0.8 & -0.338 & 1.175 & -0.211 & 0.133 & 0.209 & 55.778 & 7.872 & 1.155 & 1.960 & 0.269 & 1.023 & 1.049 & 0.691 \\
\hline 0.9 & -0.389 & 1.377 & 0.019 & 0.131 & 0.219 & 60.385 & 7.569 & 1.157 & 1.577 & 0.273 & 1.023 & 1.050 & 0.831 \\
\hline 1 & -0.519 & 1.576 & 0.276 & 0.124 & 0.229 & 65.526 & 7.286 & 1.150 & 1.364 & 0.278 & 1.023 & 1.051 & 1.000 \\
\hline
\end{tabular}

The non-inferior solutions in the Pareto frontier in Figure 26(a) are possibilities to manage the trade-off between roughness and roundness. Considering the first column of Table 12 which stores the weights $w_{1}$ related to $\operatorname{MSE}\left[\operatorname{Ron}_{t}\right]$, with $w_{2}=1-w_{1}$ related to $M S E\left[R_{a}\right]$, the engineer may set the desired weight for each quality outcome and set-up the matching $\mathbf{x}^{*}$ level.

To endorse the procedure, the NNC bi-objective optimisation results were compared with NBI and WS methods. Figure 27 shows the overlaid Pareto frontier for these three methods and the Table 13 summarizes the results considering the computational time (CPU time), number of Pareto optimal solutions obtained (Nsolutions) and the coefficient of variation of the vector of Euclidian distances between adjacent Pareto solutions $\left(C V_{\overline{\mathbf{d}}}\right)$, which measures the evenness of the solutions. To measure the CPU time for solving the $n_{s u b}=51$ with each method, the programmed routine was executed 10 times and a confidence interval for the average time was obtained with 0.95 of confidence. The CPU time, in this case, considers the initial definitions, the NNC loop, the Pareto filtering and Pareto frontier plotting. It can be observed that the NBI average time was lower than NNC and WS, however, it cannot be affirmed that the average times are statistically different due to the confidence limits. The NBI method was the only which presented difficulties in achieving the $n_{s u b}=51$ Pareto optimal solutions, presenting two dominated solutions. About the even distribution of the solutions, the NNC method presented the best results, with $C V_{\overline{\mathbf{d}}} \mathrm{NNC}=0.379$. By observing graphically, the NBI and NNC solutions were in most equal, since the NBI points denoted by '*' are almost inside the NNC symbols, denoted by 'o'. However, the two solutions which NBI returned as dominated increased the variation among the Euclidian distances of the solutions. The equality constraint in NBI sometimes may present difficulties in exploring some regions of the Pareto frontier. Besides, in the original NBI formulation, the notion of Pareto filtering is not advised. 

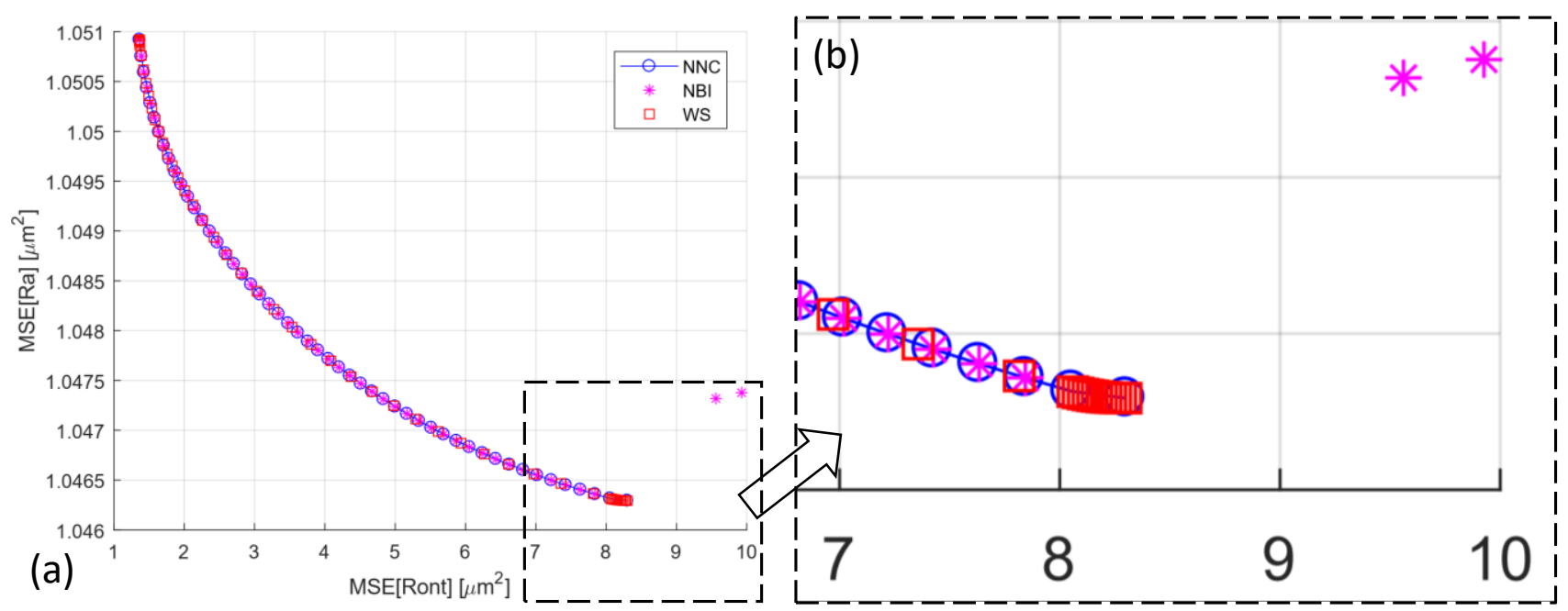

Figure 27. Pareto frontier of $M S E\left[R_{0} n_{t}\right]$ and $M S E\left[R_{a}\right]$ for WS, NBI and NNC methods (a) complete view (b) detail

Table 13. Performance of WS, NBI and NNC methods

\begin{tabular}{cccccc}
\hline Method & \multicolumn{2}{c}{ CPU Time $(\mathrm{s})$} & Nsolutions & $C V_{\overline{\mathbf{d}}}$ \\
\hline WS & 0.686 & \pm & 0.013 & 51 & 0.972 \\
NBI & 0.663 & \pm & 0.023 & 49 & 1.347 \\
NNC & 0.682 & \pm & 0.023 & 51 & 0.379 \\
\hline
\end{tabular}

Figure 28 presents the computational cost obtained for two critical parts of the multiobjective optimisation of $M S E\left[\operatorname{Ron}_{t}\right]$ and $M S E\left[R_{a}\right]$, the NNC loop in Figure 28(a), and the Pareto filtering in Figure 28(b). As explained in section 8 the complexity of the NNC loop is of liner order, $O\left(n_{s u b}\right)$, while the complexity of the Pareto filtering is of quadratic order, $O\left(n_{\text {sub }}^{2}\right)$. However, for a feasible number of desired solutions, the NNC loop will consume a higher computational time, since $n_{s u b}$ the non-linear optimisations will demand considerable higher time than $n_{\text {sub }}^{2}$ simple comparisons. For $n_{s u b}=1001$ time for the NNC loop was more than eighty times larger than the time for Pareto filtering. Considering a practical situation, to achieve $n_{s u b}=1001$ may not result in interesting results since the difference between adjacent Pareto solutions is lowermost and, consequently impracticable in a manufacturing production scenario. Consequently, considering a number of solutions feasible to represent the trade-off of the solutions in a practical scenario, the NNC loop will present higher computational time than the Pareto filtering, despite the computational complexity order of these procedures. 


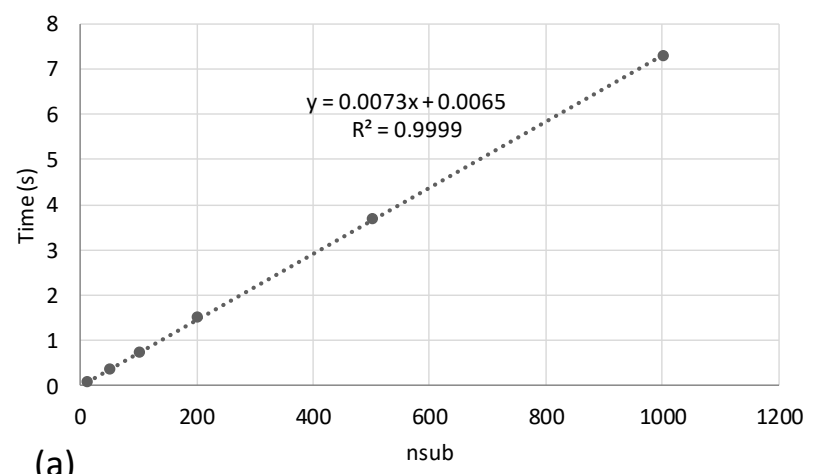

(a)

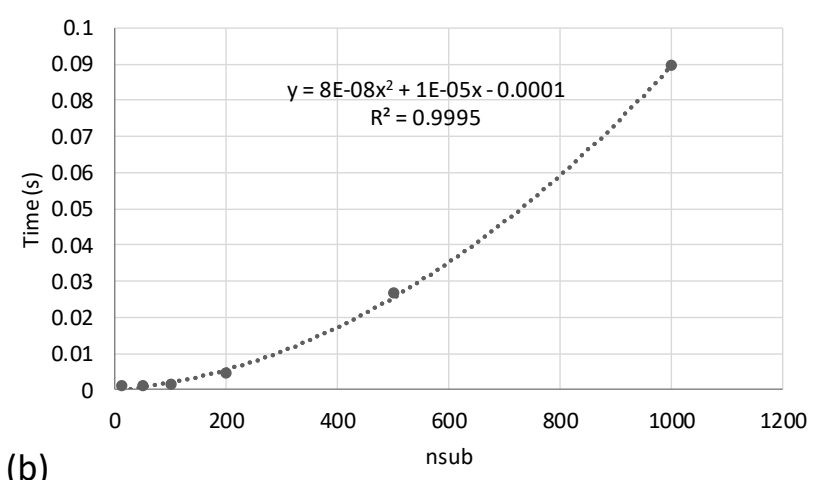

(b)

Figure 28. Computational time of: (a) NNC loop; (b) Pareto filter

Since in multi-objective optimization it is important to choose the best compromise Pareto optimal solution considering a mathematical decision criterion, it was calculated the Euclidian distance in the normalized space from each Pareto optimal solution to the utopia point $\overline{\mathbf{f}}^{\mathrm{U}}=\left[\begin{array}{ll}0 & 0\end{array}\right]^{\mathrm{T}}$, labelled $d_{j+}$. The results are also stored in Table 12. The Pareto optimal solution with lower Euclidian distance to $\overline{\mathbf{f}}^{\mathbf{U}}$ was $\mathbf{f}_{[\mathrm{w} 1=0.5]}^{*}=\left[3.759 \mu \mathrm{m}^{2} 1.048 \mu \mathrm{m}^{2}\right]^{\mathrm{T}}$, with the same weight for both functions, i.e. $w_{1}=w_{2}=0.5$. Figure 29 illustrates the Pareto frontier for $M S E\left[R_{a}\right]$ and $M S E\left[\operatorname{Ron}_{t}\right]$ in the normalized solution space with the lowest Euclidian distance from the solution $\mathbf{f}_{[\mathrm{w} 1=0.5]}^{*}$ to the utopia point $\overline{\mathbf{f}}^{\mathbf{U}}$.

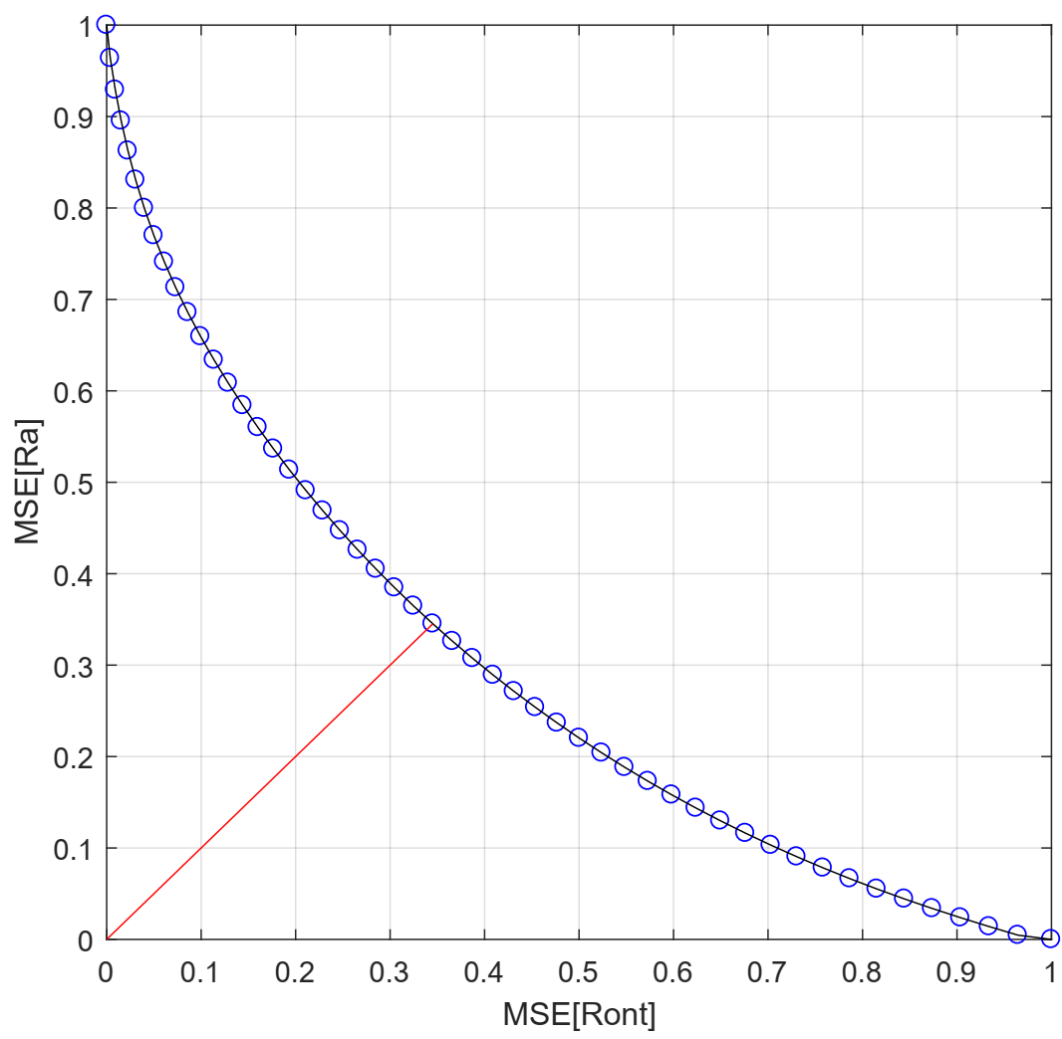

Figure 29. Pareto frontier in the normalized solution space with lowest $d_{j+}$ 
After confirming that NNC outperformed NBI in terms of the number of Pareto optimal solutions and WS in terms of evenness the Pareto optimal solutions, it is important to confirm the optimisation to assure the reduction of the variability with regard to noise factors. Therefore, the experimental together with simulated results, considering the most compromise solution, $\mathbf{f}_{[\mathrm{w} 1=0.5]=}=$ [3.759 $\left.\mu \mathrm{m}^{2} 1.048 \mu \mathrm{m}^{2}\right]^{\mathrm{T}}$, were plotted in individual and moving range (MR) control charts in Figure 30. The variability expressed by the experimental runs is due to control and noise variables, while, the variability expressed in the simulated Pareto optimal points was calculated considering the slope, i.e., the partial derivatives with regard to the noise factors $\hat{\mathbf{I}}\left(\mathbf{x}_{[w 1=0.5]}^{*}\right)=\hat{\boldsymbol{\gamma}}+\hat{\boldsymbol{\Delta}}^{\mathbf{T}} \mathbf{x}^{*}$ to express the variability in units of the measured response. The slope is suitable to express the variability, since the variance expressed in Equation 12, propagated considering the noise factors, takes into consideration the experimental error $\hat{\sigma}_{\varepsilon}^{2}$, which reflects the variance of the experimental tests in the robust modelling. Consequently, $S=\sqrt{\operatorname{Var}[\mathbf{z},[y(\mathbf{x}, \mathbf{z})]}$ always will present higher values than $\hat{\sigma}_{\varepsilon}^{2}$.

For $R_{a}$ the simulated optimal results presented mean $\bar{R}_{a}^{*}=0.254 \mu \mathrm{m}$, while for the experimental runs the mean was $\bar{R}_{a}=0.265 \mu \mathrm{m}$. With regard to the variability of $R_{a}$, the experimental runs presented $S_{\bar{R}_{a}}=0.047 \mu \mathrm{m}$, while the slope of the simulated optimal solution, which is the standard deviation propagated with regard to noise factors, was $S_{\bar{R}_{a}^{*}}=\hat{\mathbf{l}}_{\bar{R}_{a}^{*}}\left(\mathbf{x}_{[w 1=0.5]}^{*}\right)=$ $0.004 \mu \mathrm{m}$. Consequently, the most compromise Pareto optimal solution presented a lower mean $R_{a}$ besides variability reduction, as observed in Figure 30(a). 

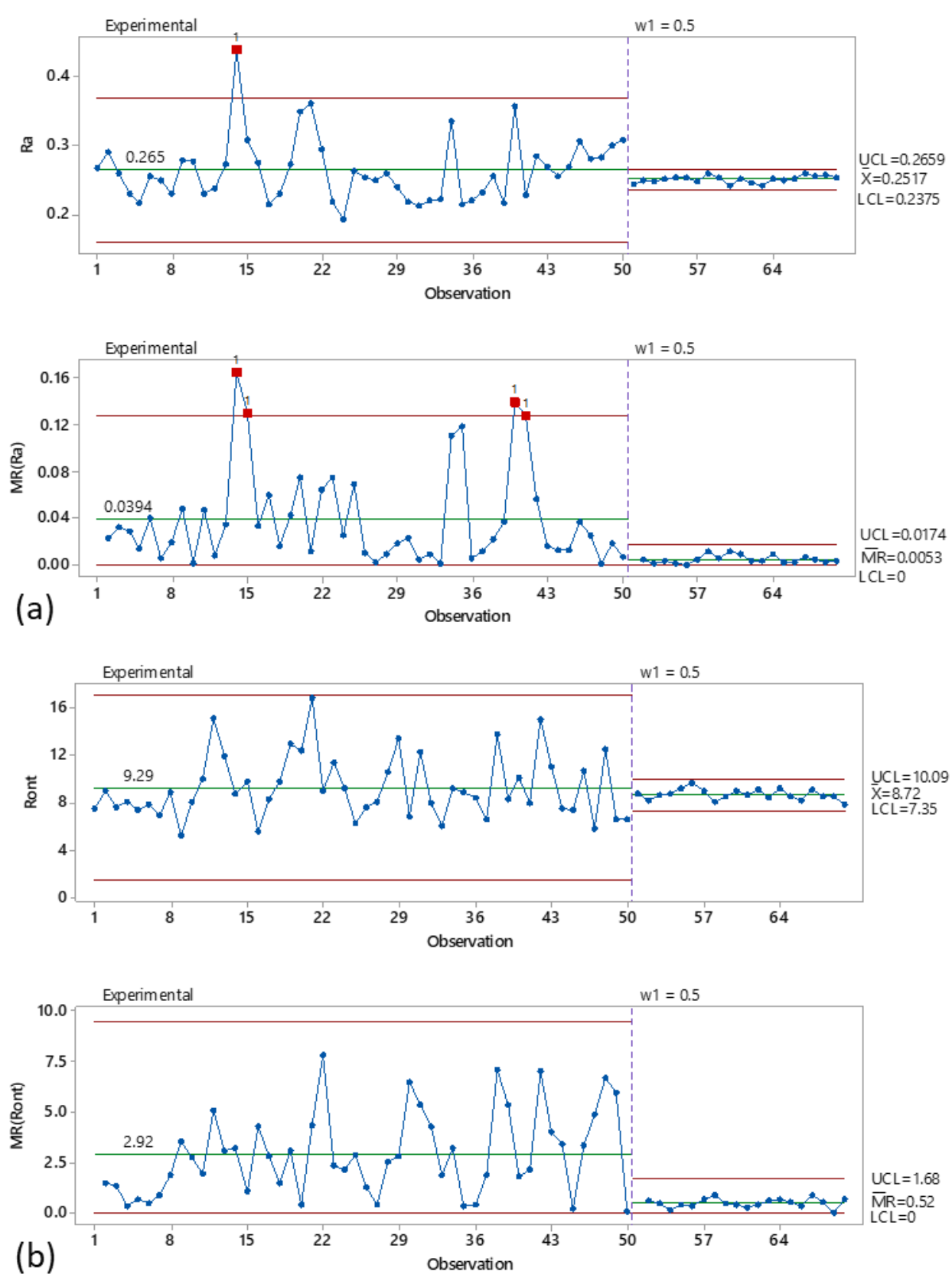

Figure 30. I/MR control charts for experimental and simulated optimal results, $\mathrm{w}_{1}=0.5$

For $\operatorname{Ron}_{t}$ the simulated optimal results presented mean $\bar{R} o n_{t}^{*}=9.29 \mu \mathrm{m}$, while for the experimental runs the mean was $\bar{R} o n_{t}=8.72 \mu \mathrm{m}$. Concerning the variability of $\operatorname{Ron}_{t}$, the experimental runs presented $S_{\bar{R} o_{t}}=2.675 \mu \mathrm{m}$, while the slope of the simulated optimal solution, which is the standard deviation propagated with regard to noise factors, was $S_{\bar{R} o n_{t}^{*}}=\hat{\mathbf{l}}_{\bar{R} o n_{t}^{*}}\left(\mathbf{x}_{[w 1=0.5]}^{*}\right)=$ $0.478 \mu \mathrm{m}$. Consequently, the most compromise Pareto optimal solution presented a lower mean $\operatorname{Ron}_{t}$ besides variability reduction, as concluded through Figure 30(b). 


\section{Conclusions}

This paper presents a methodology to the multi-objective robust optimization of borehole quality obtained by helical milling on AISI H13 hardened steel. Since hole-making in hardened material is a difficult task, the results achieved with the proposed methodology are important to attain competitive advantage in the molds and die industry. The proposed methodology for robust multi-objective modelling and optimisation may be applied in other manufacturing processes.

Experiments were carried out by following a CCD combined array considering process and noise helical milling factors. Tool overhang length, material hardness, and borehole height were considered as noise factors to achieve process factor levels insensitive with regard to noise variation.

Response models were obtained for $\operatorname{Ron}_{t}, C y l_{t}, R_{a}$, and $R_{z}$, in function of process and noise factors. The models presented good data variability explanation with $R_{a d j}{ }^{2}$ equals to $99.97 \%, 98.51 \%$, $99.45 \%$ and $97.91 \%$, respectively. The models also presented good prediction capability for future observations with $98.78 \%$ for $R o n_{t}$, and $94.48 \%, 84.81 \%$ for $R_{a}$ and $R_{z}$, except for $C y l_{t}$ with moderate predictability $58.29 \%$. These models are useful to study the process and noise effects in the outcomes.

Ward's dendrogram was used to select helical milling outcomes to be optimized without similarity. Ron ${ }_{t}$ and $C y l_{t}$ were separated in a highly correlated group of geometrical error outcomes, while $R_{a}$ and $R_{z}$ were separated in a highly correlated group of roughness outcomes. Then in each group, one variable was selected to represent the group, since the multi-objective optimisation involving high correlated outcomes may result in similar results, since the trade-off, in this case, is negligible. The outcomes $\operatorname{Ron}_{t}$ and $R_{a}$ were selected due to the best adjustment in each group and due to its importance in the characterization of geometrical error and roughness of boreholes, respectively.

Mean and variance equations were derived through RPD for all responses since they represent different performance characteristics. Mean square error optimisation was realised only for the selected responses $\operatorname{Ron}_{t}$ and $R_{a}$ as an approach to reduce bias and variance. The Pareto optimal solution with lower Euclidian distance to $\overline{\mathbf{f}}^{\mathbf{U}}$ was $\mathbf{f}_{[\mathrm{w} 1=0.5]}{ }^{*}\left[3.759 \mu \mathrm{m}^{2} 1.048 \mu \mathrm{m}^{2}\right]^{\mathrm{T}}$, with the same weight for both functions, i.e. $w_{1}=w_{2}=0.5$.

NNC multi-objective optimization method was used to achieve Pareto robust optimal solutions, giving to the experimenter several possibilities of process parameter levels to achieve desired borehole quality levels insensitive to the noise factors. NNC was applied to the optimisation of mean and variance of $\operatorname{Ron}_{t}$ and $R_{a}$ to evaluate the trade-off between mean and variance. 
The NNC was applied to the bi-objective optimisation of $M S E\left[R o n_{t}\right]$ and $M S E\left[R_{a}\right]$ to evaluate the trade-off between roughness and roundness considering the robustness with regard to noise factors. The NNC performance was compared with WS and NBI methods, considering CPU time, evenness of distributions and number of Pareto solutions. NNC presented similar CPU with regard to WS and NBI, the lowest coefficient of variation of the vector of Euclidian distances between adjacent Pareto solutions $C V_{\overrightarrow{\mathbf{d}}} \mathrm{NNC}=0.379$ resulting in the best solutions distribution and Nsolutions $_{\mathrm{NNC}}=51$, while NBI presented Nsolutions $\mathrm{NBI}_{\mathrm{N}}=49$.

The best compromise Pareto optimal solution was selected considering the Euclidian distance $d_{j+}$ of each Pareto optimal solution to the utopia point in the normalized solution space. I/MR Control charts were used to endorse the results comparing the experimental results with simulated results of the most compromise solution. The results showed that the Pareto solution assured robustness based on the noise variation.

The proposed methodology was detailed and may be applied in other manufacturing processes as a multi-objective robust modelling and optimization procedure. The obtained results may be employed in hole-making tasks of AISI H13 hardened steel assuring excellence in microgeometrical and geometrical borehole quality with robustness with regard to noise factors.

For future work, it is recommended to apply multi-objective evolutionary algorithms, such as Non-dominated Sorting Genetic Algorithm II, Multi-objective Evolutionary Algorithm Based on Decomposition, Multi-objective evolutionary particle swarm and other algorithms for solving multiobjective problems applied to robust optimisation of manufacturing process such as helical milling of AISI Hardened steel. Apart from borehole quality metrics, considered in the objective of the present work of borehole robust design, it is recommended to address other objectives of the helical milling process, such as productivity, cutting force and energy consumption outcomes.

\section{Acknowledgements}

The authors gratefully acknowledge the Brazilian National Council for Scientific and Technological Development (CNPq), the Coordination of Superior Level Staff Improvement (CAPES) and the Research Support Foundation of the State of Minas Gerais (FAPEMIG) for supporting this research. The authors gratefully acknowledge the Foundation for Science and Technology of Portugal (FCT) for supporting the project Sustainable and intelligent manufacturing by machining (FAMASI), process POCI-01-0145-FEDER-031556. The first author acknowledges CAPES for the PDSE grant, process 88881.133263/2016-01. 


\section{References}

[1] Fiorentino A. Cost drivers-based method for machining and assembly cost estimations in mould manufacturing. The International Journal of Advanced Manufacturing Technology, 2014;70(58);1437-1444.

[2] Diniz, A. E., Ferreira, J. R., \& Silveira, J. F. (2004). Toroidal milling of hardened SAE H13 steel. Journal of the Brazilian Society of Mechanical Sciences and Engineering, 26(1), 17-21.

[3] Brandão, L. C., Neves, F. O., \& Nocelli, G. C. (2011). Evaluation of hole quality in hardened steel with high-speed drilling using different cooling systems. Advances in Mechanical Engineering, 3, 1-7.

[4] Tönshoff, H. K., Spintig, W., König, W., \& Neises, A. (1994). Machining of holes developments in drilling technology. CIRP Annals-Manufacturing Technology, 43(2), 551-561.

[5] Wang, H., Qin, X., \& Li, H. (2015). Machinability analysis on helical milling of carbon fiber reinforced polymer. Journal of Advanced Mechanical Design, Systems, and Manufacturing, 9(5), 1-11.

[6] Astakhov, V. P. (2011). Machining of hard materials-definitions and industrial applications. In Machining of Hard Materials (pp. 1-32). Springer, London.

[7] Altan, T., Lilly, B., \& Yen, Y. C. (2001). Manufacturing of dies and molds. CIRP Annals, 50(2), 404-422.

[8] Brinksmeier, E., Fangmann, S., \& Meyer, I. (2008). Orbital drilling kinematics. Production engineering, 2(3), 277-283.

[9] Iyer, R., Koshy, P., \& Ng, E. (2007). Helical milling: an enabling technology for hard machining precision holes in AISI D2 tool steel. International Journal of Machine Tools and Manufacture, 47(2), 205-210.

[10] Sasahara, H., Kawasaki, M., \& Tsutsumi, M. (2008). Helical feed milling with MQL for boring of aluminum alloy. Journal of Advanced Mechanical Design, Systems, and Manufacturing, 2(6), 1030-1040.

[11] Uçak, N., \& Çiçek, A. (2018). The effects of cutting conditions on cutting temperature and hole quality in drilling of Inconel 718 using solid carbide drills. Journal of Manufacturing Processes, 31, 662-673.

[12] Shan, Y., He, N., Li, L., Zhao, W., \& Qin, X. (2011). Orbital milling hole of aerospace Al-alloy with big pitch. Transactions of Tianjin University, 17(5), 329-335.

[13] Eguti, C. C. A., \& Trabasso, L. G. (2014). Design of a robotic orbital driller for assembling aircraft structures. Mechatronics, 24(5), 533-545.

[14] Denkena, B., Boehnke, D., \& Dege, J. H. (2008). Helical milling of CFRP-titanium layer compounds. CIRP Journal of manufacturing Science and Technology, 1(2), 64-69.

[15] Olvera, D., de Lacalle, L. N. L., Urbikain, G., Lamikiz, A., Rodal, P., \& Zamakona, I. (2012). Hole making using ball helical milling on titanium alloys. Machining Science and Technology, 16(2), 173-188.

[16] Pereira, R. B. D., Brandão, L. C., de Paiva, A. P., Ferreira, J. R., \& Davim, J. P. (2017a). A review of helical milling process. International Journal of Machine Tools and Manufacture, 120, 27-48.

[17] Wang, H. Y., \& Qin, X. D. (2013). Research on the relation between cutting parameters and axial cutting force in helical milling process. In Advanced Materials Research (Vol. 690, pp. 2480-2483). Trans Tech Publications.

[18] Saadatbakhsh, M. H., Imani, H., Sadeghi, M. H., \& Farshi, S. S. (2017). Experimental study of surface roughness and geometrical and dimensional tolerances in helical milling of AISI 4340 alloy steel. The International Journal of Advanced Manufacturing Technology, 93(9-12), 40634074. 
[19] Uhlmann, E., \& Richarz, S. (2016). Twisted deep hole drilling tools for hard machining. Journal of Manufacturing Processes 24, 225-230.

[20] Box, G. E., \& Draper, N. R. (2007). Response surfaces, mixtures, and ridge analyses (Vol. 649). John Wiley \& Sons.

[21] Box, G., \& Wilson, K. (1951). On the Experimental Attainment of Optimum Conditions. Journal of the Royal Statistical Society. Series B (Methodological), 13(1), 1-45. Retrieved from http://www.jstor.org/stable/2983966

[22] Toloo, M., \& Mensah, E. K. (2018) Robust optimization with nonnegative decision variables: A DEA approach. Computers \& Industrial Engineering, In Press.

[23] Mirzapour Al-E-Hashem, S. M. J., Malekly, H., \& Aryanezhad, M. B. (2011). A multi-objective robust optimization model for multi-product multi-site aggregate production planning in a supply chain under uncertainty. International Journal of Production Economics, 134(1), 28-42.

[24] Hong, Y. Y., Beltran, A. A., \& Paglinawan, A. C. (2018). A robust design of maximum power point tracking using Taguchi method for stand-alone PV system. Applied Energy, 211, 50-63.

[25] Welch, W. J., Yu, T. K., Kang, S. M., \& Sacks, J. (1990). Computer experiments for quality control by parameter design. Journal of Quality Technology, 22(1), 15-22.

[26] Shoemaker, A. C., Tsui, K. L., \& Wu, C. J. (1991). Economical experimentation methods for robust design. Technometrics, 33(4), 415-427.

[27] Nair, V. N., Abraham, B., MacKay, J., Box, G., Kacker, R. N., Lorenzen, T. J., ... \& Phadke, M. S. (1992). Taguchi's parameter design: a panel discussion. Technometrics, 34(2), 127-161.

[28] Kackar, R. N. (1985). Off-line quality control, parameter design, and the Taguchi method. journal of Quality Technology, 17(4), 176-188.

[29] Box, G. E. P. (1985). Off-line quality-control, parameter design, and the Taguchi methoddiscussion. Journal of Quality Technology, 17(4), 189-190.

[30] Myers, R. H., Khuri, A. I., \& Vining, G. (1992). Response surface alternatives to the Taguchi robust parameter design approach. The American Statistician, 46(2), 131-139.

[31] Box, G., \& Jones, S. (1992). Designing products that are robust to the environment. Total Quality Management, 3(3), 265-282.

[32] Hosseini, S. A., Amjady, N., Shafie-khah, M., \& Catalão, J. P. (2016). A new multi-objective solution approach to solve transmission congestion management problem of energy markets. Applied Energy, 165, 462-471.

[33] Messac, A., Ismail-Yahaya, A., \& Mattson, C. A. (2003). The normalized normal constraint method for generating the Pareto frontier. Structural and multidisciplinary optimization, 25(2), 86-98.

[34] Pereira, R. B. D., Leite, R. R., Alvim, A. C., de Paiva, A. P., Ferreira, J. R., \& Davim, J. P. (2017). Multi-objective robust optimization of the sustainable helical milling process of the aluminum alloy Al 7075 using the augmented-enhanced normalized normal constraint method. Journal of cleaner production, 152, 474-496.

[35] Pereira, R. B. D., Leite, R. R., Alvim, A. C., de Paiva, A. P., Balestrassi, P. P., Ferreira, J. R., \& Davim, J. P. (2018). Multivariate robust modeling and optimization of cutting forces of the helical milling process of the aluminum alloy Al 7075. The International Journal of Advanced Manufacturing Technology, 95(5-8), 2691-2715.

[36] Tönshoff, H. K., Spintig, W., König, W., \& Neises, A. (1994). Machining of holes developments in drilling technology. CIRP Annals-Manufacturing Technology, 43(2), 551561.

[37] Iyer, R. (2006). Helical milling: an enabling technology for machining holes in fully hardened AISI D2 tool steel (Doctoral dissertation).

[38] Mishra, V., Khan, G. S., Chattopadhyay, K. D., Nand, K., \& Sarepaka, R. V. (2014). Effects of tool overhang on selection of machining parameters and surface finish during diamond turning. Measurement, 55, 353-361. 
[39] Smith, S., Winfough, W. R., \& Halley, J. (1998). The effect of tool length on stable metal removal rate in high speed milling. CIRP Annals, 47(1), 307-310.

[40] Kull Neto, H., Diniz, A. E., \& Pederiva, R. (2016). Influence of tooth passing frequency, feed direction, and tool overhang on the surface roughness of curved surfaces of hardened steel. The International Journal of Advanced Manufacturing Technology, 82(1-4), 753-764.

[41] Paiva, A. P., Campos, P. H., Ferreira, J. R., Lopes, L. G. D., Paiva, E. J., \& Balestrassi, P. P. (2012). A multivariate robust parameter design approach for optimization of AISI 52100 hardened steel turning with wiper mixed ceramic tool. International Journal of Refractory Metals and Hard Materials, 30(1), 152-163.

[42] Urbicain, G., Olvera, D., de Lacalle, L. L., Zamakona, I., \& Rodal, P. (2009, November). New Strategies For Hole Making In Ti-6Al-4V. In AIP Conference Proceedings (Vol. 1181, No. 1, pp. 361-369). AIP.

[43] Li, Z., \& Liu, Q. (2013). Surface topography and roughness in hole-making by helical milling. The International Journal of Advanced Manufacturing Technology, 66(9-12), 14151425.

[44] Petrović, G. S., Madić, M., \& Antucheviciene, J. (2018). An approach for robust decision making rule generation: Solving transport and logistics decision making problems. Expert Systems with Applications, 106, 263-276.

[45] Kuhn, A. M. (2003). Optimizing response surface experiments with noise factors using confidence regions. Quality Engineering, 15(3), 419-426.

[46] Myers, R. H., Kim, Y., \& Griffiths, K. L. (1997). Response surface methods and the use of noise variables. Journal of Quality Technology, 29(4), 429-440.

[47] Borror, C. M., Montgomery, D. C., \& Myers, R. H. (2002). Evaluation of statistical designs for experiments involving noise variables. Journal of Quality Technology, 34(1), 54-70.

[48] Griffiths, K. L. (1995). Model selection and analysis tools in response surface modeling of the process mean and variance(Doctoral dissertation, Virginia Tech).

[49] Yan, X., \& Su, X. (2009). Linear regression analysis: theory and computing. World Scientific.

[50] Weisberg, S. (2005). Applied linear regression (Vol. 528). John Wiley \& Sons.

[51] Romano, J. P., \& Wolf, M. (2017). Resurrecting weighted least squares. Journal of Econometrics, 197(1), 1-19.

[52] Vining, G. G., \& Myers, R. H. (1990). Combining Taguchi and response surface philosophies: a dual response approach. Journal of quality technology, 22(1), 38-45.

[53] Lin, D. K., \& Tu, W. (1995). Dual response surface optimization. Journal of Quality Technology, 27(1), 34-39.

[54] Ferreira, D. F. (2008) Estatística multivariada. Lavras: Editora UFLA.

[55] Cavaliere, P., Perrone, A., \& Silvello, A. (2015). FEM and multi-objective optimization of steel case hardening. Journal of Manufacturing Processes, 17, 9-27.

[56] Kitayama, S., Tamada, K., Takano, M., \& Aiba, S. (2018). Numerical optimization of process parameters in plastic injection molding for minimizing weldlines and clamping force using conformal cooling channel. Journal of Manufacturing Processes, 32, 782-790.

[57] Logist, F., \& Van Impe, J. (2012). Novel insights for multi-objective optimisation in engineering using normal boundary intersection and (enhanced) normalised normal constraint. Structural and Multidisciplinary Optimization, 45(3), 417-431.

[58] Emmerich, M. T., \& Deutz, A. H. (2018). A tutorial on multiobjective optimization: fundamentals and evolutionary methods. Natural computing, 17(3), 585-609.

[59] Das, I., \& Dennis, J. E. (1998). Normal-boundary intersection: A new method for generating the Pareto surface in nonlinear multicriteria optimization problems. SIAM Journal on Optimization, 8(3), 631-657. 
[60] Das, I., \& Dennis, J. E. (1997). A closer look at drawbacks of minimizing weighted sums of objectives for Pareto set generation in multicriteria optimization problems. Structural optimization, 14(1), 63-69.

[61] Messac, A., \& Mattson, C. A. (2004). Normal constraint method with guarantee of even representation of complete Pareto frontier. AIAA journal, 42(10), 2101-2111.

[62] Sanchis, J., Martinez, M., Blasco, X., \& Salcedo, J. V. (2008). A new perspective on multiobjective optimization by enhanced normalized normal constraint method. Structural and multidisciplinary optimization, 36(5), 537-546.

[63] Sayyaadi, H., \& Mehrabipour, R. (2012). Efficiency enhancement of a gas turbine cycle using an optimized tubular recuperative heat exchanger. Energy, 38(1), 362-375.

[64] Sun, T. H. (2009). Applying particle swarm optimization algorithm to roundness measurement. Expert Systems with Applications, 36(2), 3428-3438.

[65] Costa, D. D. D., Marques, A., \& Amorim, F. L. (2015). Hole quality and cutting time evaluation in the interpolated helical milling. International Journal of Manufacturing Research, 10(4), 313-327.

[66] Tarng, Y. S., Kao, J. Y., \& Lin, Y. S. (1997). Identification of and compensation for backlash on the contouring accuracy of CNC machining centres. The International Journal of Advanced Manufacturing Technology, 13(2), 77-85.

[67] Zain, A. M., Haron, H., \& Sharif, S. (2010). Prediction of surface roughness in the end milling machining using Artificial Neural Network. Expert Systems with Applications, 37(2), 17551768.

[68] Niaki, F. A., \& Mears, L. (2017). A comprehensive study on the effects of tool wear on surface roughness, dimensional integrity and residual stress in turning IN718 hard-to-machine alloy. Journal of Manufacturing Processes, 30, 268-280.

[69] Benardos, P. G., \& Vosniakos, G. C. (2003). Predicting surface roughness in machining: a review. International journal of machine tools and manufacture, 43(8), 833-844.

[70] Pereira, R. B. D., Hincapie, C. A. A., da Silva Campos, P. H., de Paiva, A. P., \& Ferreira, J. R. (2016). Multivariate global index and multivariate mean square error optimization of AISI 1045 end milling. The International Journal of Advanced Manufacturing Technology, 87(912), 3195-3209.

[71] Sun, D., Lemoine, P., Keys, D., Doyle, P., Malinov, S., Zhao, Q., ... \& Jin, Y. (2018). Holemaking processes and their impacts on the microstructure and fatigue response of aircraft alloys. The International Journal of Advanced Manufacturing Technology, 94(5-8), 1719-1726.

Appendices

A. Proof that the OLS results may be applied to WLS problems

The assumption of homoscedasticity of the experimental observations may be relaxed through $\operatorname{Var}(\boldsymbol{\varepsilon})=\sigma^{2} \mathbf{W}^{-1}$, with $\boldsymbol{\varepsilon}$ as the vector of error terms, where $\mathbf{W}$ is a diagonal matrix with $w_{i}$ as diagonal element. The model, in this case, can be expressed as follows [50]:

$\mathbf{Y}=\mathbf{X} \boldsymbol{\beta}+\boldsymbol{\varepsilon}, \quad \operatorname{Var}(\boldsymbol{\varepsilon})=\sigma^{2} \mathbf{W}^{-1}$ 
On the contrary, in the case of homoscedasticity, the model is presented as follows [50]:

$\mathbf{Y}=\mathbf{X} \boldsymbol{\beta}+\boldsymbol{\varepsilon}, \quad \operatorname{Var}(\boldsymbol{\varepsilon})=\sigma^{2} \mathbf{I}_{N}$

In the case of Equation A2, the estimates for $\boldsymbol{\beta}$ are obtained through OLS, i.e., $\hat{\boldsymbol{\beta}}=\left(\mathbf{X}^{\mathbf{T}} \mathbf{X}\right)^{-1} \mathbf{X}^{\mathbf{T}} \mathbf{Y}$. The problem A1 may be transformed to one solved by OLS. Let $\mathbf{W}^{1 / 2}$ be a $N \times N$ diagonal matrix with $i$-th diagonal term $\sqrt{w_{i}}$. Then, $\mathbf{W}^{-1 / 2}$ is a diagonal matrix with $i$-th diagonal term $1 / \sqrt{w_{i}}$. Therefore $\mathbf{W}^{1 / 2} \mathbf{W}^{-1 / 2}=\mathbf{I}_{N}$. The covariance matrix of $\mathbf{W}^{-1 / 2} \boldsymbol{\varepsilon}$ is [50]:

$$
\begin{aligned}
& \operatorname{Var}\left(\mathbf{W}^{1 / 2} \boldsymbol{\varepsilon}\right)=\mathbf{W}^{1 / 2} \operatorname{Var}(\boldsymbol{\varepsilon}) \mathbf{W}^{1 / 2} \\
& \operatorname{Var}\left(\mathbf{W}^{1 / 2} \boldsymbol{\varepsilon}\right)=\mathbf{W}^{1 / 2} \sigma^{2} \mathbf{W}^{-1} \mathbf{W}^{1 / 2} \\
& \operatorname{Var}\left(\mathbf{W}^{1 / 2} \boldsymbol{\varepsilon}\right)=\sigma^{2} \mathbf{W}^{1 / 2}\left(\mathbf{W}^{-1 / 2} \mathbf{W}^{-1 / 2}\right) \mathbf{W}^{1 / 2} \\
& \operatorname{Var}\left(\mathbf{W}^{1 / 2} \boldsymbol{\varepsilon}\right)=\sigma^{2}\left(\mathbf{W}^{1 / 2} \mathbf{W}^{-1 / 2}\right)\left(\mathbf{W}^{1 / 2} \mathbf{W}^{-1 / 2}\right) \\
& \operatorname{Var}\left(\mathbf{W}^{1 / 2} \boldsymbol{\varepsilon}\right)=\sigma^{2} \mathbf{I}_{N}
\end{aligned}
$$

Taking (A1) and multiplying by $\mathbf{W}^{1 / 2}[50]$ :

$$
\mathbf{W}^{1 / 2} \mathbf{Y}=\mathbf{W}^{1 / 2} \mathbf{X} \boldsymbol{\beta}+\mathbf{W}^{1 / 2} \boldsymbol{\varepsilon}
$$

Defining $\mathbf{W}^{1 / 2} \mathbf{Y}=\boldsymbol{\Omega}, \mathbf{W}^{1 / 2} \mathbf{X}=\boldsymbol{\Xi}$, and $\mathbf{W}^{1 / 2} \boldsymbol{\varepsilon}=\Gamma[50]$ :

$$
\boldsymbol{\Omega}=\boldsymbol{\Xi} \boldsymbol{\beta}+\boldsymbol{\Gamma}
$$

Through A2 $\operatorname{Var}(\boldsymbol{\Gamma})=\sigma^{2} \mathbf{I}_{N}$. Consequently, estimating $\boldsymbol{\beta}$ through OLS [50]:

$$
\begin{aligned}
& \hat{\boldsymbol{\beta}}=\left(\boldsymbol{\Xi}^{\mathrm{T}} \boldsymbol{\Xi}\right)^{-1} \boldsymbol{\Xi}^{\mathrm{T}} \boldsymbol{\Omega} \\
& \hat{\boldsymbol{\beta}}=\left(\left(\mathbf{W}^{1 / 2} \mathbf{X}\right)^{\mathbf{T}}\left(\mathbf{W}^{1 / 2} \mathbf{X}\right)\right)^{-1}\left(\mathbf{W}^{1 / 2} \mathbf{X}\right)^{\mathbf{T}}\left(\mathbf{W}^{1 / 2} \mathbf{Y}\right) \\
& \hat{\boldsymbol{\beta}}=\left(\mathbf{X}^{\mathbf{T}} \mathbf{W}^{1 / 2} \mathbf{W}^{1 / 2} \mathbf{X}\right)^{-1} \mathbf{X}^{\mathbf{T}} \mathbf{W}^{1 / 2} \mathbf{W}^{1 / 2} \mathbf{Y} \\
& \hat{\boldsymbol{\beta}}=\left(\mathbf{X}^{\mathbf{T}} \mathbf{W} \mathbf{X}\right)^{-1} \mathbf{X}^{\mathbf{T}} \mathbf{W} \mathbf{Y}
\end{aligned}
$$

The estimates in Equation A6 are the WLS estimates. 
B. Homoscedasticity and normality test results

Since it was used a fractional factorial design, the homoscedasticity tests of multiple comparison and Levene were performed considering groups of parameters, as exposed in Table B.1. As can be observed in the most part of the results presented in the Table B.1, the homoscedasticity null hypothesis can be rejected, since $p$-value $<0.05=\alpha$. Consequently, the WLS estimates for the response models may be more efficient to deal with non-constant error terms.

Table B.1. Homoscedasticity tests for the responses

\begin{tabular}{cccc}
\hline & $f_{z a}, f_{z t}, v_{c}, l_{t o}$ & $f_{z a}, f_{z t}, v_{c}, h_{d}$ & $f_{z a}, f_{z t}, v_{c}, h_{d}$ \\
\hline \multirow{2}{*}{$C y l_{t}$} & $0.019^{*}$ & 0.165 & 0.060 \\
& $0.262^{* *}$ & 0.081 & 0.270 \\
Ron $_{t}$ & 0.018 & 0.000 & 0.000 \\
& 0.013 & 0.010 & 0.080 \\
$R_{a}$ & 0.000 & 0.000 & 0.000 \\
& 0.022 & 0.000 & 0.010 \\
$R_{z}$ & 0.000 & 0.000 & 0.000 \\
\multirow{2}{*}{$*$ multiple-comparisons test p-value; $* *$ Levene test p-value } \\
\hline
\end{tabular}

The Anderson-Darling normality tests results and probability plots of residuals are presented. The residuals were obtained through the response models estimates of OLS. The WLS regression was performed considering the inverse of squared residuals and Romano and Wolf (2017) assured that non-normality of errors in WLS estimates is not prejudicial in the efficiency of the estimates. showed that WLS is more efficient than OLS also with non-normal error terms. Figure B.1 presents the normality test for the residuals of $R_{o n}$ and $C y l_{t}$, and Figure B.2 for $R_{a}$ and $R_{z}$.
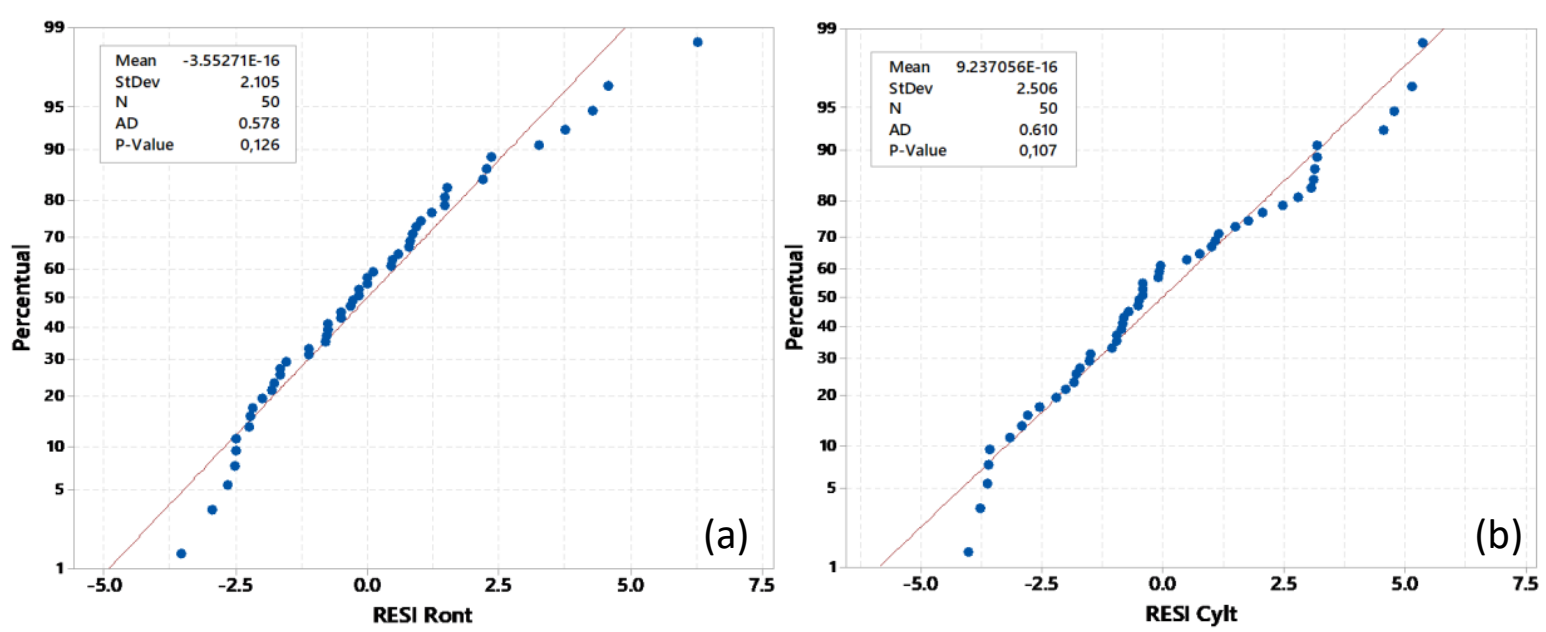
Figure B.1. Normality test for (a) $\operatorname{Ron}_{t}$ and (b) Cyl $l_{t}$
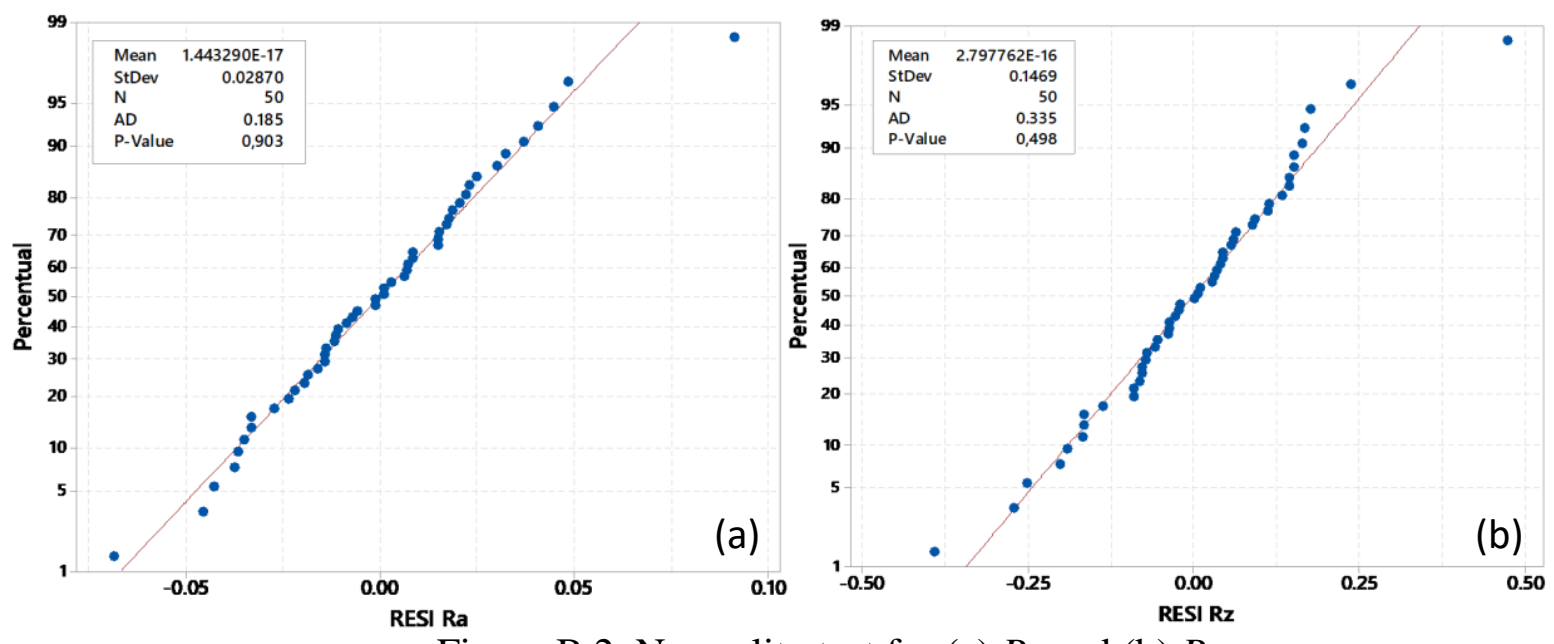

Figure B.2. Normality test for (a) $R_{a}$ and (b) $R_{z}$ 